\title{
Three-dimensional, high resolution, computerized ionospheric tomographic imaging and computational modeling of an artificial ionospheric cavity
}

\author{
Craig A. Selcher \\ West Virginia University
}

Follow this and additional works at: https://researchrepository.wvu.edu/etd

\section{Recommended Citation}

Selcher, Craig A., "Three-dimensional, high resolution, computerized ionospheric tomographic imaging and computational modeling of an artificial ionospheric cavity" (2007). Graduate Theses, Dissertations, and Problem Reports. 2588.

https://researchrepository.wvu.edu/etd/2588

This Dissertation is protected by copyright and/or related rights. It has been brought to you by the The Research Repository @ WVU with permission from the rights-holder(s). You are free to use this Dissertation in any way that is permitted by the copyright and related rights legislation that applies to your use. For other uses you must obtain permission from the rights-holder(s) directly, unless additional rights are indicated by a Creative Commons license in the record and/ or on the work itself. This Dissertation has been accepted for inclusion in WVU Graduate Theses, Dissertations, and Problem Reports collection by an authorized administrator of The Research Repository @ WVU.

For more information, please contact researchrepository@mail.wvu.edu. 


\title{
Three-Dimensional, High Resolution, Computerized Ionospheric Tomographic Imaging and Computational Modeling of an Artificial Ionospheric Cavity
}

\author{
Craig A. Selcher \\ Dissertation submitted to the Eberly College of Arts and Sciences at West Virginia \\ University in partial fulfillment of the requirements for the degree of Doctor of \\ Philosophy in Physics
}

\author{
Mark Koepke, Ph.D., Chair \\ Earl Scime, Ph.D. \\ Art Weldon, Ph.D. \\ Boyd Edwards, Ph.D. \\ Paul Bernhardt, Ph.D. \\ Department of Physics \\ Morgantown, West Virginia \\ 2007
}

Keywords: Ionosphere, Physics

Copyright 2007 Craig A. Selcher 


\section{Abstract \\ Three-Dimensional, High Resolution, Computerized Ionospheric Tomographic Imaging and Computational Modeling of an Artificial Ionospheric Cavity Craig A. Selcher}

Tomographic techniques use line integral measurements to reconstruct local values of the measured parameter. These techniques have been applied to the ionosphere by using radio transmissions to measure the integral of electron density between a satellite and a chain of ground-based receiving stations. The resultant reconstructions form a two-dimensional map of the electron density in the plane of the satellite / receiver chain. Insufficient quantity of receivers and not having a complete range of accessible look angles with the available receivers are responsible for the nonuniqueness in the reconstructions. The limited look angle problem can be alleviated by making use of alternative data sources such as incoherent scatter radars (ISR) that provide information on the vertical structure of the electron density. The non-optimal receiver placement problem can be alleviated through the use of sophisticated reconstruction algorithms. The computerized ionospheric tomography (CIT) technique has recently been used to image the artificially modified ionosphere above the Arecibo Observatory (AO) HF heating facility. A total of nine radio beacon receivers forming a three by three matrix were deployed across the entire island of Puerto Rico. The arrangement maximizes the likelihood that several of the receiver-to-satellite lines of sight would intersect the heated region of the ionosphere. Several satellite passes associated with such an intersection occurred, and the resultant tomographic inversions 
indicate the existence of an electron density cavity approximately 45 kilometers in latitudinal extent. The reduction of electron density in this cavity was typically on the order of $20 \%$. The experimental observations were supported by theoretical work using the open-source SAMI2 ionospheric model. Methods were developed to model both the ohmic heating of the electrons and the suprathermal heating caused by nonlinear effects. Modeled ohmic heating values of $941 \mathrm{~K} / \mathrm{s}$ match the observed heated temperature profiles. Modeled suprathermal electrons effects on the vibrational temperature of $\mathrm{N}_{2}$ indicate a vibrational / translation temperature differential of $308^{\circ} \mathrm{K}$. 


\section{DEDICATION}

The author wishes to dedicate this work to his wife Trish, for her support, encouragement and patience throughout this long process. 
Table of Contents

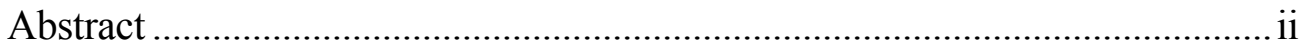

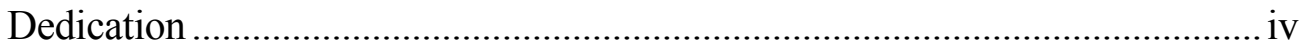

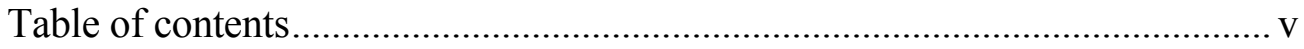

List of Figures ….......................................................................................... vi

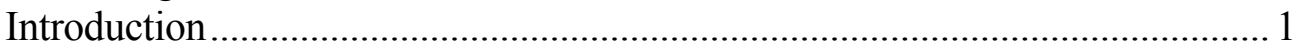

Section C: Context of the problem: The Earth's ionosphere............................. 5

Section D: Experimental Method: Computerized Ionospheric Tomography 18

Section E: Experimental Method: Incoherent Scatter Radar..........................2 29

Section F: Theoretical Method: Modified SAMI2 Ionospheric Model ......... 31

Section G: Phenomenon being Investigated:

Large -Scale Artificially Generated Ionospheric Cavities..... 35

Section H: Description of Experiment ...................................................... 38

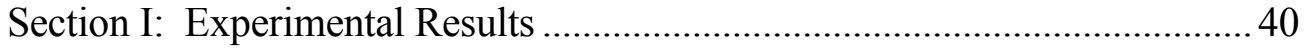

Section J: Discussion of Experimental Results............................................ 46

Section K: Discussion of Theoretical Results.............................................. 49

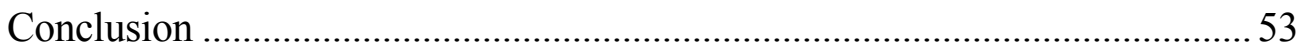

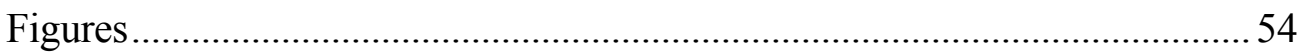

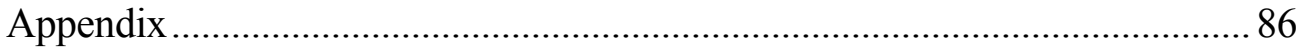




\section{Figure List}

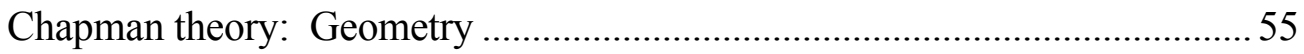

Chapman theory: Hydrostatic gas and Intensity profile .................................56

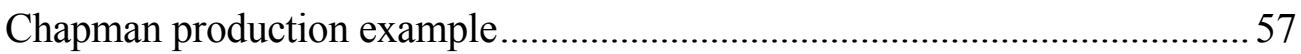

Multi-spectrum Chapman: 1/e Intensity Height ........................................... 58

Multi-spectrum Chapman: Examples...........................................................59

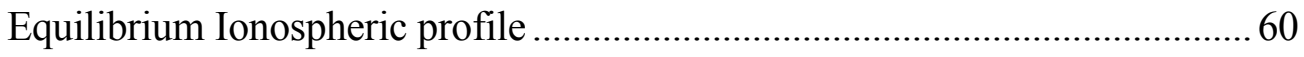

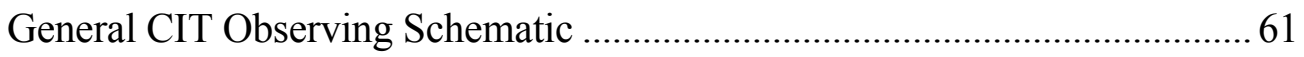

HF Ionospheric Modification Schematic ....................................................... 62

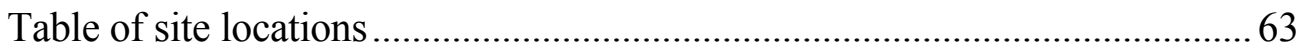

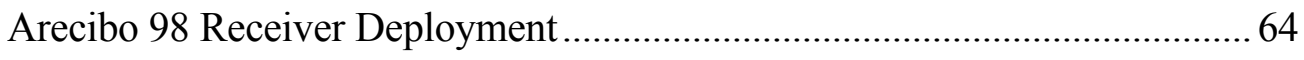

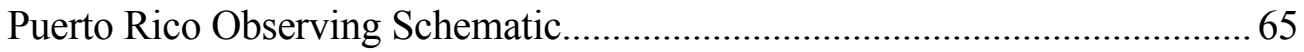

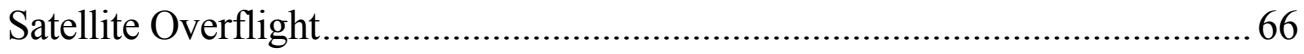

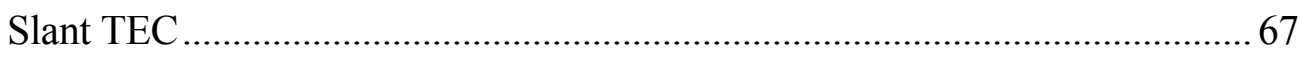

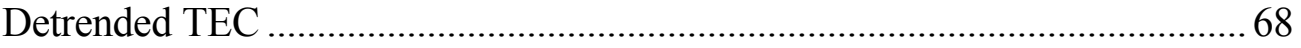

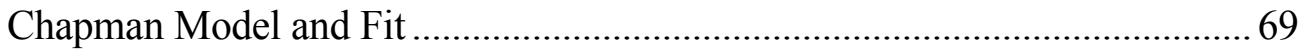

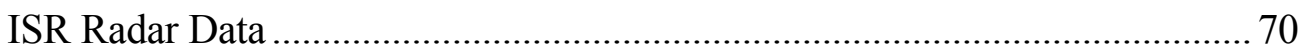

Chapman model based reconstructions

027_0337 Western Chain Reconstruction .............................................. 71

027 0337 Central Chain Reconstruction ................................................ 72

027_0337 Eastern Chain Reconstruction................................................. 73

ISR data based reconstructions

027_0337 Western Chain Reconstruction ............................................... 74

027 0337 Central Chain Reconstruction ................................................ 75

027_0337 Eastern Chain Reconstruction.................................................. 76

ISR Temperature Data and $\mathrm{n}_{\mathrm{e}}$ Scaling Factor .............................................. 77

SAMI2 Background Electron Temperature ................................................... 78

SAMI2 Heated Electron Temperature ………................................................ 79

SAMI2 Change in Electron Temperature Due to Heating .............................. 80 


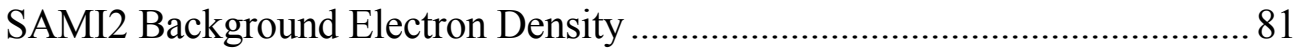

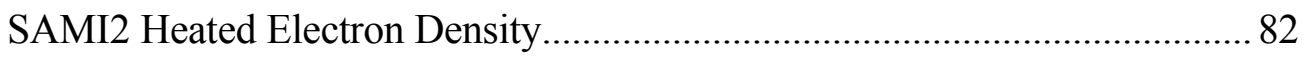

SAMI2 Change in Electron Density Due to Heating ….................................. 83

SAMI2 Change in Electron Density, Heating and Reaction Rate Effects ....... 84

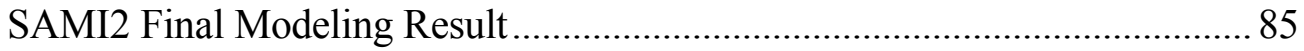

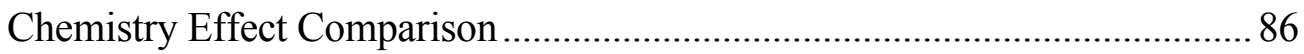




\section{B. Introduction}

\section{B.1. What is Tomography?}

The computerized ionospheric tomography (CIT) technique involves the transmission of a beacon radio signal through the ionosphere, typically from a satellite to a ground-based receiving system. The phase delay introduced into the signal by passage through the complex dielectric plasma medium of the ionosphere is extracted and used to determine the total electron count (TEC) between the transmitting and receiving locations. This TEC value and the ray-path geometry associated with each value are then incorporated into a reconstruction algorithm which forms twodimensional maps of electron density. ${ }^{1,2}$

The implementation of the ionospheric tomographic technique has traditionally been applied exclusively to naturally occurring, large-scale ionospheric structures. ${ }^{3,4,5 \text {, }}$

\footnotetext{
1 Bernhardt, P.A., R.P. McCoy, K.F. Dymond, J.Ml Picone, R. R. Meier, F. Kamalabadi, D.M. Cotton, S. Chakrabarti, T.A. Cook, J.S. Vickers, A.W.Stephan, L. Kersely, S.E. Pryse, I.K. Walker, C.N Mitchell, P.R. Straus, H. Na, C. Biswas, G.S. Bust, G.R. Kronschnabl, T.D. Raymund., Two-dimensional mapping of the plasma density in the upper atmosphere with computerized ionospheric tomography (CIT), Phys. Plas., 5, 2010-2021, May 1998.

${ }^{2}$ Austen, J.R., S.J. Franke, and C.H. Liu, Ionospheric imaging using computerized tomography, Radio Science, 23, 299-307, 1988.

${ }^{3}$ Kersley, 1., Heatyon, J.A.T., S.E. Pryse, and T.D. Raymund, Experimental ionospheric tomography with ionosonde input and EISCAT verification, Anns. Geophys., 11, 1064-1074, 1993.

${ }^{4}$ Pryse, S.E., and L. Kersley, A preliminary experimental test of ionospheric tomography, J. Atmos. Terr. Phys. 54, 1007-1912, 1992.

${ }^{5}$ Pryse, S.E., L. Kersley, D.L. Rice, C.D. Russell, and I.K. Walker, Tomographic imaging of the ionospheric mid-latitude trough, Anns. Geophys., 11, 144-149, 1993.
} 
$6,7,8,9,10,11,12$ The work discussed here makes use of the small-scale spatial resolution available via the employment of relatively high data rate tomographic receiver systems. These systems record differential phase between two radio beacon signals at a rate sufficient to resolve any TEC structure of interest ${ }^{13}$ to a degree comparable to one kilometer.

\section{B.2. Experiment description}

During the January 1998 Arecibo Observatory high power HF ionospheric heating campaign, an array of CIT receivers was deployed on the island of Puerto Rico, forming a dense, high resolution, three by three matrix. This arrangement allows, for the first time, three parallel reconstruction planes, each oriented approximately along a line of longitude, which are suitable for investigating changes in cavity extent both along and across the magnetic field lines. Measurements of TEC using the differential

\footnotetext{
${ }^{6}$ Raymund, T.D., J.R. Austen, S.J. Franks, C.H. Liu, J.A. Klobuchar, and J. Stalker, Application of computerized tomography to the investigation of ionospheric structures, Radio Science, 25, 771-789, 1990.

${ }^{7}$ Raymund, T.D., Y. Bresler, D.N. Anderson, and R.E. Daniell, Model-assisted ionospheric tomography: A new algorithm, Radio Science, 29, 1493-1512, 1994.

${ }^{8}$ Pryse, S.E., L. Kersley, M.J. Williams, and I.K. Walker, The spatial structure of the dayside ionosheric trough, Ann. Geophysicae, 16, 1169-1179, 1998

${ }^{9}$ Cook, J.A., S. Close, An investigation of TID evolution observed in MACE '93 data, Ann. Geophysicae, 13, 1320-1324, 1995

${ }^{10}$ Pryse, S.E. C.N. Mitchell, J.A.T. Heaton, L. Kersley, Travelling ionospheric disturbances imaged by tomographic techniques, Ann. Geophysicae, 13, 1325-1330, 1995

${ }^{11}$ Bust, G.S.; Gaussiran, T.L., II; Coco, D.S., Ionospheric observations of the November 1993 storm, J. Geophys. Res., 102, 14293-14304, 1997

${ }^{12}$ Kronschnabl, G.R.; Bust, G.S.; Cook, J.A.; Vasicek, C.J., Mid-America computerized ionospheric tomography experiment (MACE '93), Radio Science, 30, 105-108, 1995

${ }^{13}$ Leitinger, R., Data from orbiting navigation satellites for tomographic reconstructions, Int. J. Imaging Syst. Technol., 5, 86-96, 1994
} 
Doppler technique were made during both heater-on and heater-off periods. To supplement this closely spaced data set, the Applied Research Lab (ARL) of the University of Texas at Austin made available the data from a chain of CIT receivers deployed throughout the Caribbean region. Vertical profiles of electron density were obtained by use of the Incoherent Scatter Radar (ISR) at the Arecibo Observatory, and were used both to determine the absolute value of TEC and to form empirical initial conditions for the reconstructions.

\section{B.3. Data discussion and Summary}

Reconstructions generated using code developed at the Naval Research Laboratory (NRL) by the author (see Appendix A) clearly indicate the existence of electron density cavities consistent with previous measurements ${ }^{14,15,16}$ and demonstrate the applicability of CIT to the imaging of artificial structures formed in an high-frequency (HF) heated ionosphere. In addition, since the differential phase technique solely measures the electron density, CIT may be of assistance in resolving the electron density / temperature ambiguity present in ISR measurements. ${ }^{17} \mathrm{We}$ considered limitations to the CIT results and found that by using closely-spaced 3receiver chains we can resolve and investigate artificially generated structures which are

\footnotetext{
${ }^{14}$ Hansen, J.D., Morales, G.J., Duncan, L.M., Dimonte, G., Large-scale HF-induced ionospheric modifications: Experiments, JGR, 97, 113-122, 1992

${ }^{15}$ Bernhardt, P. A., C. A. Tepley, and L. M. Duncan, Airglow enhancements associated with plasma cavities formed during ionospheric heating experiments, J. Geophys. Res., 94, 9071-9092, 1989.

${ }^{16}$ Duncan, L.M., J.P. Sheerin, R.A. Behnke, Observations of ionospheric cavities generated by highpower radio waves, Phys. Rev. Lett., 61, 239-242, 1988

${ }^{17}$ Sulzer, M.P., A radar technique for high range resolution incoherent scatter autocorrelation function measurements utilizing the full average power of klystron radars, Radio Sci., 21, 1033, 1986
} 
of a much smaller dimension than the naturally occurring ionospheric structures which had been previously imaged using the CIT technique. 
C. Context of the problem: The Earth's ionosphere.

C.1. Defined and described

The Earth's ionosphere is typically taken to be that region of the upper atmosphere where plasma is present and has sufficient density to affect the propagation of electromagnetic waves, particularly those in the radio frequency range. This region begins near $80 \mathrm{~km}$ in altitude and extends to and merges with the magnetosphere at roughly $1000 \mathrm{~km}$ altitude. The upper and lower boundaries of the ionosphere are not well-defined, and shift according to diurnal and seasonal variations and other processes. By convention, the ionosphere is divided into a number of layers, denoted by the letters D, E, and F. The D-region extends from roughly 50 to $90 \mathrm{~km}$, the E-region extends from 90 to $150 \mathrm{~km}$, and the F-region comprises the remaining portion of the ionosphere from $150 \mathrm{~km}$ to $1000 \mathrm{~km}$ Once again, these boundaries are not well-defined, and vary as ionospheric conditions change. ${ }^{18}$

The $\mathrm{D}$ region is very weakly ionized, highly collisional, and primarily composed of molecular oxygen ions $\left(\mathrm{O}_{2}{ }^{+}\right)$and nitric oxide ions $\left(\mathrm{NO}^{+}\right)$. For the purposes of this study, the sole effect of the D region is to absorb a negligibly small portion of the beacon radio signal. Assuming that the beacon frequency is much greater than the electron-neutral collision frequency, and neglected effects due to the geomagnetic field, the absorption coefficient is, $\kappa=1.15 \times 10^{-3} \frac{N v}{f^{2}}$

18 Rishbeth, H., O.K. Garriot, Introduction to Ionospheric Physics, New York: Academic Press, 1969 
in units of decibels per kilometer, and where $N$ is the electron density, $v$ is the electronneutral collision frequency, and $f$ is the beacon frequency. Typical D-region values of $v\left(10^{8} / \mathrm{s}\right)$ and $N\left(10^{3}\right.$ electrons $\left./ \mathrm{cm}^{3}\right)$ result in $\kappa=5.1 \times 10^{-8}$. The total attenuation through the D-region is therefore approximately $1.53 \times 10^{-7} \mathrm{~dB}$, and is clearly negligible.

The $\mathrm{E}$ region, extending from $90 \mathrm{~km}$ to $150 \mathrm{~km}$, is also composed primarily of molecular oxygen ions $\left(\mathrm{O}_{2}^{+}\right)$and nitric oxide ions $\left(\mathrm{NO}^{+}\right)$. Typical E region peak electron concentrations are on the order of $10^{5}$ electrons $/ \mathrm{cm}^{3}$. The time constants associated with the various chemical processes that occur at E region heights are short enough that transport can be neglected, and photochemistry dominates.

The F-region, extending from $150 \mathrm{~km}$ to $1000 \mathrm{~km}$, is predominantly populated with oxygen ions $\left(\mathrm{O}^{+}\right)$. At the uppermost portion of the ionosphere near $1000 \mathrm{~km}$ altitude, helium ions $\left(\mathrm{He}^{+}\right)$and hydrogen ions $\left(\mathrm{H}^{+}\right)$are the dominant species. ${ }^{19}$ Typical $\mathrm{F}$ region peak densities are on the order of $10^{6}$ electrons $/ \mathrm{cm}^{3}$. The $\mathrm{F}$ region is frequently conceptually divided into three sub-regions based upon the predominant physical processes in each sub-region. The lowest region is dominated by photochemistry, has negligible diffusion, and is known as the F1 region. The region near the peak electron concentration profile corresponds to a transition from photochemical dominance to diffusion dominance, and is called the F2 region. The upper F region, dominated by diffusion, is called the topside of the ionosphere. It is clear that due to both its spatial extent and high concentration, the bulk of the ionospheric plasma lies in the F-region.

19 Kelley, M.C., The Earth's ionosphere, Academic Press, 1989 
Since the total electron content (TEC) is a measure of the integrated electron density, the most significant contribution to the measured TEC is from the F-region.

The primary production mechanism for both the $\mathrm{F}$ and $\mathrm{E}$ regions is photo ionization by the absorption of solar photons by the neutral atmospheric gas. A useful analytic model which serves to quantitatively describe the core features of this mechanism is known as the Chapman model, and is described in detail in section C.3. The steady-state ionospheric electron concentration profile is determined by the combination of production, primarily the photo ionization described in section C.3, and loss through recombination and transport, and is described in section C.4. The resultant mathematical model of the ionosphere, which combines the primary qualitative features of these mechanisms and captures the major characteristics of a quiet mid-latitude ionosphere, is described in section C.5.

\section{C.2. The mid-latitude ionosphere at Arecibo}

The ionosphere at the latitude of the Arecibo Observatory exemplifies the midlatitude ionosphere. The geomagnetic dip angle (the angle at which the Earth's magnetic field passes through the ionosphere) is $50^{\circ}$ with respect the horizontal. This allows for some degree of freedom for the upward and downward flow of charged particles, since that flow is constrained to be parallel or antiparallel to the magnetic field. The geomagnetic latitude is $34^{\circ}$, which is far from both the auroral oval at $70^{\circ}$ geomagnetic latitude and the energetic precipitating particles to be found there. Consequently, the mid-latitude ionosphere is relatively "quiet" compared to the 
tumultuous auroral (or near auroral) ionosphere at the latitude of many of the other ionospheric research facilities. ${ }^{20}$

\section{C.3. Chapman Production Function Theory}

The analytic approach used in this work to provide a theoretical description of the generation mechanism of the ionosphere is known as Chapman theory. Several simplifying assumptions are made to make the problem tractable. The first assumption is that the sole source of ionization is photo ionization by absorption of solar radiation. This radiation is modeled as having a single value for the absorption cross section, and the absorbing atmospheric gas is assumed to be composed of a single constituent which is planar, horizontally stratified, and in hydrostatic equilibrium. ${ }^{21}$

Hydrostatic equilibrium results in a vertical profile of neutral gas density which obeys:

$n_{n}(h)=n_{o} \exp \left(-\frac{\left(h-h_{o}\right)}{H_{n}}\right)$

where $n_{o}$ is the neutral density at reference height $\mathrm{h}_{\mathrm{o}}$ and $H_{n}=k T_{n} / m_{n} g$ is the exponential scale height of the neutral gas. This exponential decrease in neutral density with height is a result of balancing the effect of gravity on the gas with the thermal pressure within the gas.

Chapman theory avoids dealing directly with the complex nature of the radiative transfer of energy through the neutral gas by folding all photon absorption details into

\footnotetext{
${ }^{20}$ Sojka, J.J., Ionospheric physics, Rev. of Geophys., Supp., 1166-1186, 1991

${ }^{21}$ Chapman, S., The theoretical height distribution of band-absorbed solar radiation, Proc Phys Soc (London), 51, 93-109
} 
the photon absorption cross section $\sigma$ and using an ionization probability $\eta$ to convert the energy absorbed into the number of electrons released from the neutral gas. The rate of electron production $q$ can therefore be given by $q=\eta \sigma n_{n} I$,

where $\eta$ is the ionizing efficiency (photoelectrons produced per photon absorbed), $\sigma$ is the cross section of absorption, $n_{n}$ is the neutral gas density, and $I$ is the radiation intensity.

The geometry of this situation is shown in Figure 1a. As the radiation is absorbed, its intensity will fall off with distance along the line of sight s,

$-\frac{d I}{d s}=\sigma n_{n} I$

as can be seen in Figure 1b. Note that the electron production is proportional to the rate at which radiation is absorbed, i.e., $q=-\eta \frac{d I}{d s}$

The electron production rate $q$ reaches a maximum when, according to C.3.2

$\frac{d q}{d s}=\eta \sigma\left(I \frac{d n_{n}}{d s}+n_{n} \frac{d I}{d s}\right)=0$

Combining the trigonometric relation between line-of-sight path length $s$ and height $h, \frac{d h}{d s}=-\cos (\chi)$, and the hydrostatic expression for $n_{n}$ (C.3.1), yields, $\frac{1}{n_{n}} \frac{d n_{n}}{d s}=\frac{\cos (\chi)}{H_{n}}$

Substitution of C.3.5 in C.3.4 shows that for the location of maximum photoelectron production, 
$\sigma H_{n} n_{m} \sec (\chi)=1$

where the subscript $m$ indicates the neutral density measured at the maximum of electron production. This can be written in simplified form as $\sigma N_{n m}=1$, where $N_{n m}=\int_{\infty}^{s_{m}} n d s$ is the integrated neutral density from infinity to the location of the

production maximum at $s_{m}$.

Integrating C.3.3 to obtain $I$ as a function of $s$, and extending our use of $N_{n s}$ to indicate the neutral density integral, we have,

$$
\begin{aligned}
& \frac{d I}{I}=d(\ln I)=-\sigma n_{n} d s, \\
& \ln \left(\frac{I(s)}{I_{\infty}}\right)=-\sigma \int_{\infty}^{s} n_{n} d s=-\sigma N_{n s}, \\
& I(s)=I_{\infty} \exp \left(-\sigma N_{n s}\right) .
\end{aligned}
$$

Combining C.3.2, C.3.6 and C.3.7 leads to a peak production rate of

$$
q_{m}=\eta \sigma n_{m} I_{m}=\eta \sigma \frac{I_{\infty} \exp (-1)}{\sigma H_{n} \sec (\chi)}=\frac{\eta I_{\infty} \cos (\chi)}{H_{n} \exp (1)}
$$

since $\sigma N_{n m}=1$ at the production peak. The height at which this maximum occurs can be determined by inserting the hydrostatic equation into (C.3.6),

$$
\sigma H_{n} n_{o} \exp \left(-\frac{\left(h_{m}-h_{o}\right)}{H_{n}}\right) \sec (\chi)=1
$$

and solving for $h_{m}$

$$
h_{m}=h_{o}-H_{n} \ln \left(\frac{\cos (\chi)}{\sigma H_{n} n_{o}}\right)
$$


From C.3.7 and once again using the relationship between $s$ and $h$, we can determine $I(h)$,

$$
I(h)=I_{\infty} \exp \left[-\sigma n_{o} H_{n} \sec (\chi) \exp \left(-\frac{\left(h-h_{o}\right)}{H_{n}}\right)\right],
$$

and subsequently the production rate $q$ as a function of height,

$$
q(h)=\eta \sigma n_{n} I=\eta \sigma n_{o} I_{\infty} \exp \left[-\frac{\left(h-h_{o}\right)}{H_{n}}-n_{o} \sigma H_{n} \sec (\chi) \exp \left(-\frac{\left(h-h_{o}\right)}{H_{n}}\right)\right] .
$$

From C.3.8, $\eta I_{\infty}=q_{m} \frac{\exp (1) H_{n}}{\cos (\chi)}$, and using the value of $h_{m}$ from C.3.9,

$$
q=q_{m} \exp \left[1+\frac{\left(h_{m}-h\right)}{H_{n}}-\exp \left(\frac{\left(h_{m}-h\right)}{H_{n}}\right)\right]
$$

Substituting $z=\frac{\left(h-h_{m}\right)}{H_{n}}$ results in the simple expression for the Chapman production function $q$, $q=q_{m} \exp [1-z-\exp (-z)]$

A representative example of the Chapman production function is shown in Figure 2. One of the primary variations of the actual photoionization profiles from the simplest Chapman theory is due to the multi-component nature of the neutral atmospheric gas and the wavelength dependent absorption coefficient of the incoming solar radiation. These two factors lead to complexity in the absorption and production profile compared to the simple profile depicted in Figure 2.

A portion of this complexity can be accounted for by separating the absorption components into three bands. Figure $3 \mathrm{a}$ is a plot of the height at which incoming solar 
radiation will be reduced to $1 / \mathrm{e}$ of its original intensity, as a function of the wavelength of the incoming radiation. From equation C.3.7, it can be seen that this corresponds to the height of the production maximum. Wavelengths below $20 \mathrm{~nm}$ and above $80 \mathrm{~nm}$ are primarily responsible for the production of the E region. The wavelength band between $20 \mathrm{~nm}$ and $80 \mathrm{~nm}$ is primarily responsible for the production of the $\mathrm{F}$ region. Figure $3 \mathrm{~b}$ shows the Chapman photoionization production rates for each of these bands as functions of height. The long-term structure of the ionosphere is to a large degree a combination of the photoionization production described in this section and the loss and transport mechanisms described in section C.4 
C.4. Loss, Transport, and the Equilibrium Ionosphere

There are several recombination process which are responsible for the bulk of the loss of oxygen ions, the predominant ion in the F region. These process are given by:

$$
\begin{aligned}
& \mathrm{O}+h v \rightarrow \mathrm{O}^{+}+e \\
& \mathrm{O}^{+}+\mathrm{N}_{2} \rightarrow \mathrm{NO}^{+}+\mathrm{N} \\
& \mathrm{NO}^{+}+e \rightarrow \mathrm{N}+\mathrm{O} \\
& \mathrm{O}^{+}+\mathrm{O}_{2} \rightarrow \mathrm{O}_{2}^{+}+\mathrm{O} \\
& \mathrm{O}_{2}^{+}+e \rightarrow \mathrm{O}+\mathrm{O}
\end{aligned}
$$

Equation C.4.1 represents direct radiative recombination, and occurs slowly enough to be ignored. Equations pairs C.4.2, C.4.3 and C.4.4, C.4.5 are ion-atom interchange reactions followed by dissociative recombination of the resultant molecular ion. These two reaction pairs are the primary recombination mechanisms for the oxygen ions in the $\mathrm{F}$ region.

For the regions where photochemistry dominates, the E and F1 regions, transport is ignored and the continuity equation for species $j$ becomes

$\frac{d n_{j}}{d t}=P_{j}-L_{j}$

where $\mathrm{P}_{\mathrm{j}}$ is the production rate and $\mathrm{L}_{\mathrm{j}}$ is the loss rate. For steady-state conditions, $\frac{d n_{j}}{d t}=0$, hence $P_{j}=L_{j}$ and the loss rate due to recombination is proportional to the product of the densities of the two recombining species. If it is assumed that one ion 
species dominates (which is reasonably valid for oxygen ions in the F1 and F2 regions), then the density of that ion is equal to the electron density, and the recombination loss term can be expressed as $P_{j}=L_{j}=\alpha n_{e}^{2}$, where $\alpha$ is the ion-electron recombination rate. Using the Chapman production function C.3.13, we have

$$
\begin{aligned}
& \alpha n_{e}^{2}=q_{m} \exp [1-z-\exp (-z)] \\
& n_{e}=\left(\frac{q_{m}}{\alpha}\right)^{1 / 2} \exp \left\{\frac{1}{2}[1-z-\exp (-z)]\right\} .
\end{aligned}
$$

In the $\mathrm{E}$ region, the recombination-rate coefficient $\alpha$ is relatively independent of altitude and concentrations of minor constituents, and the electron density profile can be expected to follow Equation C.4.7 in a relatively uncomplicated manner. At F1 region heights, where photochemistry still dominates the physics and diffusion is not a significant factor, but the recombination-rate coefficient is not independent of altitude and concentrations of minor constituents, the result is more complicated.

An expression which illustrates this more complicated behavior can be derived by again assuming a steady state and equating the production function C.3.13 for oxygen ions with the loss terms for those ions shown in equations C.4.2 - C.4.5:

$n_{O+}=\frac{q_{O+}}{\alpha_{N 2} n\left(N_{2}\right)+\alpha_{O 2} n\left(O_{2}\right)}$,

where $\alpha_{\mathrm{N} 2}$ and $\alpha_{\mathrm{O} 2}$ are the chemical rate constants associated with the ion-atom interchange/dissociative recombination reactions from equations C.4.2-C.4.5, and $\mathrm{n}\left(\mathrm{N}_{2}\right)$ and $\mathrm{n}\left(\mathrm{O}_{2}\right)$ are the densities of the molecular nitrogen and oxygen respectively. 
The numerator in equation C.4.8 is the production function C.3.13 for oxygen ions, and decreases exponentially with altitude since it is directly proportional to the exponentially decreasing atomic oxygen density (recall from figures $3 a$ and $3 b$ that the peak of the production function is at around $150 \mathrm{~km}$ ). However, the recombination rates $\alpha_{\mathrm{N} 2}$ and $\alpha_{\mathrm{O} 2}$ are directly proportional to the molecular oxygen $\left(\mathrm{O}_{2}\right)$ and nitrogen $\left(\mathrm{N}_{2}\right)$ densities, which fall off at an even greater exponential rate due to their greater molecular weight. This leads to a resultant exponential increase in oxygen ion concentration with height throughout the $\mathrm{F} 1$ region until diffusion begins to play a role at $\mathrm{F} 2$ region heights.

In the topside ionosphere, above the $\mathrm{F} 2$ region, diffusion dominates photochemistry, and the ion concentration follows a diffusive equilibrium profile. The classical diffusive equilibrium equation is given by

$\frac{1}{n_{j}} \frac{\partial n_{j}}{\partial r}=-\frac{1}{H_{j}}-\frac{1}{T_{j}} \frac{\partial T_{j}}{d r}$

where $n_{j}$ is the density of species $j, T_{j}$ is the temperature of species $j$, and

$$
H_{j}=\frac{2 k T_{j}}{m_{j} g}
$$

is the scale height for species $j$. C.4.9 is easily solved with the assumption of an isothermal ionosphere, $\frac{\partial T_{j}}{d r}=0$, and the result is

$$
n_{j}=n_{j 0} \exp \left[-\left(r-r_{0}\right) / H_{j}\right]
$$


This results in a density profile which exponentially decreases with height in the diffusion dominated topside region. Figure 4 illustrates the competing processes which contribute to the equilibrium density profile of the ionospheric $\mathrm{F}$ region.

\section{C.5. The Five-Parameter "Chapman" Model}

The characteristics of the multiply exponential profile described in section C.4 are reasonably well represented by modifying the Chapman production function by both increasing the scale height and the peak altitude to account for the variation from a direct photochemical profile. The scale height then represents an effective scale height which includes diffusive effects, and the peak height provides indication of the crossover point between photochemistry and diffusion dominance. Other modification can be made to the simple Chapman function to account for the multiple species present in the ionosphere. The five-parameter Chapman model used in this work includes both a small linear and a quadratic variation of the scale height $H_{n}$ with altitude above the density peak. This results in a more accurate description of the topside of the ionosphere where the primary constituents are hydrogen and helium rather than oxygen, since their smaller molecular weight results in a more gradual density decrease compared to oxygen. This five-parameter model is given by:

$$
n=n_{\max } \exp \left[1+\frac{\left(h_{\max }-h\right)}{H_{0}}-\exp \left(\frac{\left(h_{\max }-h\right)}{H_{0}}\right)\right] ; \quad \mathrm{h}<\mathrm{h}_{\max }
$$


$n=n_{\max } \exp \left[1+\frac{\left(h_{\text {max }}-h\right)}{H_{0}+H_{1}\left(h_{\text {max }}-h\right)+H_{2}\left(h_{\text {max }}-h\right)^{2}}-\exp \left(\frac{\left(h_{\max }-h\right)}{H_{0}+H_{1}\left(h_{\text {max }}-h\right)+H_{2}\left(h_{\max }-h\right)^{2}}\right)\right]$ $; \mathrm{h}>=\mathrm{h}_{\max }$

where $\mathrm{H}_{0}$ is the unadjusted scale height, $\mathrm{H} 1$ is the linear contribution to the effective scale height and $\mathrm{H}_{2}$ is the quadratic contribution to the effective scale height. 


\section{Experimental Method: Computerized Ionospheric Tomography}

\section{D.1. Total Electron Content}

D.1.a. Differential phase

We arrive at a two-dimensional map of electron density in an altitude-latitude plane by measuring the differential phase between two beacon signals transmitted by a single satellite and received by an array of receivers. Differential phase is a technique which measures the line-integral of electron density by comparing the phases of two mutually coherent radio signals which are transmitted simultaneously but at two separate frequencies. ${ }^{22,23}$ For the Navy Navigation Satellite System (NNSS, or "Transit") satellites used in these experiments, the beacon frequencies are $150 \mathrm{MHz}$ and $400 \mathrm{MHz}$ (each $-80 \mathrm{ppm}){ }^{24}$ Naturally, the relative phase increases as the two signals propagate, and so as the transmitter-receiver distance evolves, the measured phase varies in a predictable fashion.

If, however a dielectric medium with a frequency-dependent dielectric function exists along the path, then a relative phase shift is introduced between the two signals. This relative phase shift reflects the details of the electron density along the path, since for high frequency waves in plasmas, the ions are virtually static and the dielectric properties of the medium are characterized by the electron motion. The measured phase shift is an accumulation of the effects integrated along the line of sight.

\footnotetext{
${ }^{22}$ Garriot, O.K., A.V. DaRosa, W.J. Ross, Electron content obtained from Faraday rotation and phase path length variations, Journal of Atmospheric and Terrestrial Physics, 32, 705-727, 1970

${ }^{23}$ Leitinger, R., G.K. Hartmann, F.J. Lohmar, E. Putz, Electron content measurements with geodetic doppler receivers, Radio Science, 19, 789-797, 1984

${ }^{24}$ Newton, R.R., The Navy Navigation satellite system, Space Res, 7, 735-763, 1967
} 
The phase accumulated by a radio wave as it passes through a dielectric medium is given by, ${ }^{25}$

$\Phi=2 \pi \int \frac{d s}{\lambda}=\frac{2 \pi}{\lambda_{0}} \int \mu d s$, in radians

where $\lambda$ is the wavelength, $\lambda_{o}$ is the free space wavelength, and $\mu$ is the dielectric constant,

$\mu^{2}=1-\left(\frac{e^{2}}{4 \pi^{2} \varepsilon_{o} m_{e}}\right)\left(\frac{N}{f^{2}}\right)=1-80.6\left(\frac{N}{f^{2}}\right)$.

Assume $N / f^{2}$ is small, and by expansion,

$\mu=1-40.3\left(\frac{N}{f^{2}}\right)$

The measured phases for two beacon signal can then be described by,

$\Phi_{1} \lambda_{1}=\int \mu_{1} d s=\int d s-\frac{40.3}{f_{1}^{2}} \int N d s=S_{1}-\frac{40.3}{q_{1}^{2} f_{o}^{2}} N_{T}$
$\Phi_{2} \lambda_{2}=\int \mu_{2} d s=\int d s-\frac{40.3}{f_{2}^{2}} \int N d s=S_{2}-\frac{40.3}{q_{2}^{2} f_{o}^{2}} N_{T}$

where $\lambda$ is the free space wavelength for the beacon signal, $q$ is the ratio of the frequency of the beacon signal to the reference frequency $f_{o}, S$ is the free space path length for the beacon signal, and $N_{T}$ is the integrated electron density along the beacon signal path. Assuming that refractive effects are negligible leads to $S_{1}=S_{2}$, and using the relation $\lambda_{1}=f_{1} c=q_{1} f_{o} c$, the phase difference is given by

${ }^{25}$ Davies, K., Ionospheric Radio, London: Peter Peregrinus Ltd., 1990 


$$
\begin{aligned}
& \Delta \Phi=\frac{\Phi_{2}}{q_{2}}-\frac{\Phi_{1}}{q_{1}}=\frac{40.3 N_{T}}{f_{o} c}\left(\frac{1}{q_{1}^{2}}-\frac{1}{q_{2}^{2}}\right), \text { in cycles, and } \\
& \Delta \Phi=\frac{80.6 \pi N_{T}}{f_{o} c}\left(\frac{1}{q_{1}^{2}}-\frac{1}{q_{2}^{2}}\right), \text { in radians },
\end{aligned}
$$

Solving for $\mathrm{N}_{\mathrm{T}}$, the Total Electron Content (TEC) yields

$$
N_{T}=\frac{\left(f_{o} c \Delta \Phi\right)}{80.6 \pi\left(\frac{1}{q_{1}^{2}}-\frac{1}{q_{2}^{2}}\right)}
$$

and this can be expressed as

$$
N_{T}=\alpha \Delta \Phi \text { where } \alpha=\frac{f_{o} c}{80.6 \pi\left(\frac{1}{q_{1}^{2}}-\frac{1}{q_{2}^{2}}\right)} \approx 6.21 \times 10^{14} \frac{\text { electrons }}{\mathrm{m}^{2} \cdot \text { radian }} \text { for the Transit beacon }
$$

for which $f_{o}=50 \mathrm{MHz}, q_{1}=3$ and $q_{2}=8$.

As an example, the differential phase that would be observed for the signals from the beacon onboard the Transit satellites (transmitting at $150 \mathrm{MHz}$ and $400 \mathrm{MHz}$ ) after passing through one kilometer of plasma with a density of $10^{5}$ electrons $/ \mathrm{cm}^{3}$ (a typical F-layer peak density) would be 0.161 radians.

\section{D.1.b. Geophysical constraints and limitations}

D.1.b.i. Phase ambiguity

The fact that $\Phi_{o}=m(2 \pi)+\Phi_{o}$ is an ambiguity that introduces an uncertainty into the measured signal at both the beginning of measurement and at each reacquisition. Reacquisition occurs sometime after the phase lock loop between the receiver and the incoming radio wave is lost, which happens occasionally in the real- 
world environment. Typically data is discarded when the phase lock is lost if the satellite has traveled a significant distance during the loss (e.g. greater than $20 \mathrm{~km}$ or 2 sec). For short periods of phase lock loss, interpolated data may be generated and substituted for the missing data.

\section{D.1.b.ii. Offset uncertainty}

Upon initial acquisition of the satellite at the horizon, there exists an uncertainty in the value of the received phase, and consequently the absolute value of TEC. This uncertainty amounts to an offset, the value of which is adjusted to match either a modelbased assumption that is made about the ionosphere or a direct empirical measurement made by a supplemental diagnostic. ${ }^{26,27,28}$ This uncertainty in initial phase means that the measured TEC will be a relative value rather than an absolute value until the offset is determined independently.

\section{D.1.b.iii. Refractive effects}

Refractive effects on the ray path of the beacon signal due to the undisturbed background ionosphere are negligibly small, since the frequency of lowest component of the beacon signal, at $150 \mathrm{MHz}$, is much greater than the maximum ionospheric plasma frequency during these experiments of approximately $5 \mathrm{MHz}$. Assuming a nominal, spherically symmetric, electron-density profile results in an angular deflection

\footnotetext{
${ }^{26}$ Austen, J. R., and S. Chalasani, Integrating ionosonde data into computerized ionospheric tomography, Proceedings of the International Beacon Satellite Symposium, Abserwyth Wales, July 1994.

${ }^{27}$ Heaton, J.A.T., S.E. Pryse, L. Kersley, Improved background representation, ionosonde input and independent verification in experimental ionospheric tomography, Anns. Geophys., 13, 1297-1302, 1995.

${ }^{28}$ Page, D.L.; Austen, J.R., Comparison of tomographic and conventional methods for resolution of the constant of integration, Radio Science, 32, 1645-56, 1997
} 
of the apparent direction of the signal from the true direction of the signal of less than one-hundredth of a degree. Thus refractive bending of the beacon signal due to the natural ionosphere can be ignored.

The bending of the beacon signal ray path due to the horizontal gradients of electron density along the edges of the large-scale artificially-generated cavity which is the focus of this work, and is described in detail in section G, can be derived from equation D.1.4. This effect is known as wedge refraction, and the bending angle which results is expressed by $\delta=\frac{40.3}{f^{2}} \frac{\partial N_{T}}{\partial x}$

The maximum horizontal gradient in the TEC for this experiment (seen in Figure 16c) is $6.176 \times 10^{10} \mathrm{~m}^{-2} \mathrm{~m}^{-1}$, resulting in a bending angle $\delta$ of $1.1 \times 10^{-4}$ radians for the $150 \mathrm{MHz}$ component of the beacon signal. The lateral deviation at the ground due to this bending over the $1000 \mathrm{~km}$ propagation path is approximately 50 meters, far less than the reconstruction pixel size and therefore having no significant impact.

\section{D.2. Computerized Ionospheric Tomography explained}

A single line-integral measurement of a structured medium is incapable of resolving that structure. However, sufficient sampling over a complete range of angles throughout the medium can be synthesized to form a reconstructed image of the structure. Insufficient sampling introduces a non-uniqueness to the image and contributes to the generation of numerical artifacts in the reconstructed image. In our case, where all sampling of the ionosphere is from lines originating on the ground and 
aimed upward, we have an ill-posed problem. ${ }^{29}$ In the near future, satellite-to-satellite integration paths will make horizontal sampling possible, dramatically improve vertical resolution, and eliminate the non-uniqueness, ${ }^{30,31}$ but presently this capability is absent. Present options to minimize the non-uniqueness of the image involve either the use of alternate measurement techniques to augment the TEC measurements, the use of a model to restrict the structure geometry, or both. Both a TEC-derived Chapman model and supplementary measurements of the electron vertical profile data using the ISR at the Arecibo Observatory have been used in this work

\section{D.3. Geometric Requirements}

To reconstruct two-dimensional structure, the paths associated with the line averages should share the same plane and should intersect. Sampling the same plane from different perspectives not only gives the variation of TEC for each line but also specifies where along each line the variations occur. The sampling of the differential phase of the received beacon signal at discrete times selects specific ray paths used in the reconstruction process. The resolvability of the structure depends on the density of intersections and the range of angles of the ray paths in a given sub-region. ${ }^{32}$ For the application of tomography to ground-based beacon receivers, the horizontal resolution

\footnotetext{
${ }^{29}$ Yeh, K.C. and T.D. Raymund, Limitations of ionospheric imaging by tomography, Radio Science, 26, 1361-1380, 1991.

${ }^{30}$ Bernhardt, P.A., K.F. Dymond, J.M. Picone, D.M. Cotton, S. Chakrabarti, T.A. Cook, and J.S. Vickers, Improved radio tomography of the ionosphere using EUV/optical measurements from satellites, Radio Science, 32, 1965-1972, 1997

${ }^{31}$ Bernhardt, P.A., C.A. Selcher, S. Basu, G. Bust, S. Reising, Atmospheric Studies with the Tri-Band Beacon Instrument on the COSMIC Constellation, TAO, 11, 291-312, 2000

${ }^{32}$ Biswas, C., H. Na, Resolution and coverage analysis for ionospheric tomography, Radio Science, 35, 905-920, 2000
} 
depends predominantly on the distance between neighboring receiver sites, whereas the vertical resolution depends predominantly on the overall extent of the array. Since a specific sub-region is reconstructed using ray paths from different receivers taken at various times, the assumption of a steady state ionosphere, and its structure, is implied for the duration of a given satellite pass. The reconstruction of a three dimensional structure requires multiple linear arrays, each resolving a different plane.

Ideally, each array of receivers should be strictly one dimensional and parallel to the ground track of each satellite pass. Due to variations within the ensemble of satellite passes and due to geographical and political constraints in choices of receiver sites, this ideal configuration is not achievable in real-world deployments. Violating these assumptions results in site-to-site differences in receiver-satellite sight angles and consequently the sampling plane of each receiver is not coplanar with the others in the chain. Variance from this guideline is acceptable so long as the site-to-site deviation is much less than the scale of the ionospheric structure under investigation. For this work, the site-to-site variance was less than a few kilometers, and the scale size of the heated volume being studied was on the order of fifty kilometers, satisfying the criteria for the chain being sufficiently collinear.

\section{D.4. Generalized observing scheme}

Figure 5 shows a generalized array of 7 receiver sites and a family of sight lines from each one. Notice that the region of maximum density of line intersections occurs above the central receiver sites. Within this region, the intersection density has a maximum at an altitude that depends on the distance between neighboring receiver 
sites. The regions above the edge receivers suffer from both low intersection-point density and narrow angle range associated with the intersecting lines. The region beyond the last receiver site suffers so much from these effects that the image in that region is assigned a minimal accuracy.

\section{D.5. Methodology}

The reconstruction of the ionospheric structure in a given plane with this method takes place on a grid superposed onto the region of interest, which extends between the satellite path and the receiver array. This region is centered around the F layer at approximately $300 \mathrm{~km}$, an altitude where the overwhelming percentage of plasma exists. Boundaries are chosen at $100 \mathrm{~km}$ and $600 \mathrm{~km}$ altitude to ensure that all pertinent TEC information is included. Boundaries on the north and south are set to be just outside of the northernmost and southernmost receiver sites, respectively.

The mesh size of the reconstruction grid is chosen to balance computational efficiency and the desire to resolve the details of the plasma structure in the ionosphere. A $5 \mathrm{~km}$ mesh size was chosen in order to clearly resolve the artificially generated cavity (expected to be on the order of $40 \mathrm{~km}$ ), to take advantage of the high-density spatial deployment of the beacon receivers, and to fully utilize the $50 \mathrm{~Hz}$ data-sampling rate of the 1502 DS receivers. The confidence in the electron density value assigned to each mesh cell improves with each additional ray path having a distinctly different angle through the cell. For a given mesh cell, the number of intersecting ray paths representing distinctly different intersection angles is approximately the number of receiver sites in the array. Therefore the confidence can be limited either by the 
available budget in receivers and their deployment or by the geographic extent of the receiver site array, or both. In the case reported here, the limitation was the small size of the island of Puerto Rico on which the Arecibo Observatory is located, and the lack of any other nearby islands to the north or south of Puerto Rico.

\section{D.6. Algorithm}

Tomographic techniques use line integral measurements to reconstruct local values of the measured parameter. This requires a large number of measurements to be taken for many paths and angles that pass through the object under study. In this case, the object under study is the ionosphere, and the parameter being measured is the electron density. A number of different tomographic methods exist, and a finite-series expansion method was chosen for this work due to its adaptability to a multitude of geometries and conditions. ${ }^{33,34,35}$

Let $\mathrm{G}(\mathrm{r}, \phi)$ be the local electron density in the satellite-receiver image plane, where $r$ is the distance from the center of the Earth, and $\phi$ is the angle of latitude. If $p_{i}$ is defined as the path for the $i$ th line-integral, then we have for the measured line integral $t_{i}$ (the collected TEC)

$$
t_{i}=\int_{p_{i}} G(r, \phi) d s, \quad \mathrm{i}=1,2,3,, N p
$$

\footnotetext{
${ }^{33}$ Raymund, T.D., Ionospheric tomography algorithms, International Journal of Imaging Systems and Technology, 5, 75-85, 1994

${ }^{34}$ Raymund, T.D., Comparisons of several ionospheric tomography algorithms, Ann Geo, 13, 1254-1262, 1995

${ }^{35}$ Censor, Y, Finite series-expansion reconstruction methods, Proc IEEE, 71, 409-419, 1983
} 
where $\mathrm{P}$ is the number of paths. We employ a finite-series expansion using $N_{b}$ basis functions $b_{j}(r, \phi)$ to describe the function $\mathrm{G}$,

$G(r, \phi) \approx \sum_{j=1}^{N_{b}} x_{j} b_{j}(r, \phi) d s=\sum_{j=1}^{N_{b}} x_{j} \int_{p_{i}} b_{j}(r, \phi) d s=\sum_{j=1}^{N b} D_{i j} x_{j}$,

where $D_{i j}=\int_{p_{i}} b_{j}(r, \phi)$, and $x_{j}$ are the weighting factors for each $b_{j}$.

Inserting this into (D.6.1) results in:

$t_{i}=\sum_{j=1}^{N_{b}} D_{i j} x_{j}$

and in matrix notation:

$\overrightarrow{\mathrm{T}}=\stackrel{\leftrightarrow}{\mathbf{D}} \overrightarrow{\mathrm{X}}$

For this work the image area is divided into pixels which are related to the set of $N_{b}$ basis functions according to,

$b_{j}(r, \phi)=\left\{\frac{1, \text { if }(r, \varphi) \text { is inside the } \mathrm{jth} \text { pixel }}{0, \text { otherwise }}\right.$.

In this case, $N_{b}$ becomes the number of pixels and the weighting factors $x_{j}$ represent the electron density in the $j$ th pixel.

The task is now to solve (D.6.2), where the array $X$ contains the unknown electron density values, the array $T$ contains the measured TEC values, and the matrix $D$ can be determined by evaluation of the ray path and pixel geometry. The iterative technique chosen for this work is a modified version of the row-action method known as the algebraic reconstruction technique (ART). At each iteration $k$ in this method, the difference between the measured values of $T$ and the computed values of $T^{k}$ is 
calculated using the current solution for $X^{k}$. This difference is used to obtain a correction to $X^{k}$, and this correction leads to $X^{k+1}$. After many iterations the corrections become insignificant and the interim solutions converge to a final solution for the matrix X.

$X^{k+1}=X^{K}+\lambda_{k}\left[\frac{t_{i}-\sum_{j=1}^{N b} D_{i j} x_{j}^{k}}{\sum_{j=1}^{N b} D_{i j} D_{i j}}\right] \boldsymbol{D}_{\boldsymbol{i}}$

where $D_{i}$ is the $i$ th row of $D, i=k \bmod N_{p}$, and $\lambda_{k}$ are relaxation parameters, in this case taken to be the same value for all $k$.

The ART requires a starting point for the electron density map $X$ to start the iteration sequence. Two approaches to form the initial map were used in this work. A Chapman model was fit to the collected TEC data, and used both to determine the absolute value of the TEC and as a source for the electron density map in the algorithm. Another approach was to use the data from the ISR (described in section E), when available, to determine the absolute value of TEC and to generate the initial electron density map for the ART algorithm. 


\section{E. Experimental Method: Incoherent Scatter Radar}

The tomographic approach used in this work makes use of empirical supplementary data to alleviate the limited-angle problem. The Arecibo Observatory includes an incoherent scatter radar (ISR) which provides the vertical profile of electron density. The incoherent (or Thomson) scatter technique takes advantage of the fact that electrons scatter electromagnetic waves. The scattering cross section, $\sigma_{\mathrm{e}}=4 \pi r_{e}{ }^{2}$, where $r_{e}$ is the effective electron radius, is extraordinarily small, on the order of $10^{-28} \mathrm{~m}^{-2}$, but modern radar technology has made detection of the scattered signal possible. Since the ionosphere is a plasma medium and not a simple electron gas, both the spectrum and the amplitude of the scattered signal are complicated by the interaction of the electrons with the constituent ions.

The received power is given by ${ }^{25}$

$\rho_{r}=\frac{C \sigma N \rho_{t} a}{R^{2}}$

where $N$ is the electron density, $\sigma$ is the effective scattering cross section, $a$ is the antenna aperture, $\rho_{t}$ is the transmitted power, and $C$ is a calibration factor which takes into account such things as feed losses, side lobes, and local geometry.

The effective cross section is given by

$$
\sigma=\frac{\sigma_{e}}{\left(1+\frac{T_{e}}{T_{i}}\right)}
$$

where $\sigma_{\mathrm{e}}$ is the Thompson scatter cross section, $T_{e}$ is the electron temperature, and $T_{i}$ is the ion temperature. 
The ratio of received power to transmitted power can then be given by

$$
\frac{\rho_{r}}{\rho_{t}}=\frac{C a N \sigma_{e}}{R^{2}\left(1+\frac{T_{e}}{T_{i}}\right)} .
$$

This illustrates the dependence of the received power on both the electron density and the electron-ion temperature ratio. Since ionospheric modification makes changes in both of these quantities, additional analysis is required. Traditionally, the spectrum and the autocorrelation function of the received ISR signal have been used to obtain estimates of $T_{e} / T_{i}$, but this is complicated by the complex nature of that spectrum due to wave-particle interactions and the disturbances created during ionospheric heating. ${ }^{36}$

ISR electron density profiles can be calibrated from either the known characteristics of the radar system, requiring detailed determination of the calibration factor $C$, or by comparison with to $\mathrm{f}_{\mathrm{o}} \mathrm{F}_{2}$ (the frequency associated with the maximum electron density in the ionosphere's $F_{2}$ layer) which can be accurately determined by a nearby ionosonde. The ISR data used in this report has been calibrated using the latter technique.

\footnotetext{
${ }^{36}$ Buneman, O., Scattering of radiation by the fluctuations in a nonequilibrium plasma, J. Geophys. Res., $67,2050,1962$
} 
F. Theoretical Method: Modified SAMI2 Ionospheric Model

\section{F.1 The SAMI2 model}

The ionospheric model used in this work is one that has been developed at the Naval Research Laboratory (NRL) by Dr.s Joe Huba and Glenn Joyce, and is known as SAMI2 is Another Model of the Ionosphere (SAMI2). SAMI2 models the mid and low-latitude ionospheric plasma along geomagnetic flux tubes extending from the northern hemisphere to the southern hemisphere, using an offset tilted dipole coordinate system. These flux tubes are considered equipotentials. ${ }^{37}$

SAMI2 solves the ion continuity and momentum (including ion inertia along the field line) equations for seven ion species $(\mathrm{H}+, \mathrm{He}+, \mathrm{N}+, \mathrm{O}+, \mathrm{N} 2+, \mathrm{NO}+$, and $\mathrm{O} 2+)$ and electrons, and the thermal balance equations for $\mathrm{H}+, \mathrm{He}+, \mathrm{O}+$, and electrons.

Ion Continuity Equation

$\frac{\partial n_{i}}{\partial t}+\nabla \cdot\left(n_{i} V_{i}\right)=P_{i}-L_{i} n_{i}$

Ion Momentum Equation:

$$
\frac{\partial V_{i}}{\partial t}+V_{i} \cdot \nabla V_{i}=\frac{1}{p_{i}} \nabla P_{i}+\frac{e}{m_{i}} E+\frac{e}{m_{i} c} V_{i} \times B+g-v_{i n}\left(V_{i}-V_{n}\right)-\sum_{j} v_{i j}\left(V_{i}-V_{j}\right)
$$

Electron Momentum Equation:

$$
0=\frac{1}{n_{e} m_{e}} \nabla P_{e}-\frac{e}{m_{e}} E-\frac{e}{m_{e} c} V_{e} \times B
$$

Ion Temperature Equation:

\footnotetext{
${ }^{37}$ Huba, J.D., Joyce, G., Fedder, J. A., Sami2 is Another Model of the Ionosphere (SAMI2): A new lowlatitude ionosphere model, J. Geophys. Res., 105, 23035-23053, 2000
} 


$$
\frac{\partial T_{i}}{\partial t}+V_{i} \cdot \nabla T_{i}+\frac{2}{3} T_{i} \nabla \cdot V_{i}+\frac{2}{3 n_{i} k} \nabla \cdot Q_{i}=Q_{i n}+Q_{i i}+Q_{i e}
$$

Electron Temperature Equation:

$$
\frac{\partial T_{e}}{\partial t}-\frac{2}{3 n_{e} k} b_{s}^{2} \frac{\partial}{\partial s} k_{e} \frac{\partial T_{e}}{\partial d s}=Q_{e n}+Q_{e i}+Q_{p h e}
$$

The ionospheric plasma is taken to be charge neutral, and so the electron density is equal to the sum of the ion densities. SAMI2 incorporates twenty-one chemical reactions, along with recombination between the electrons and all seven ion species.

These reactions provide the loss and production terms in the ion continuity equations. The neutral species are specified using the empirical models NRLMSISE00 and HWM93.

\section{F.2 Theoretical Approach to Ionospheric Heating}

A two-stage approach has been used. Initially, an iterative method was implemented by assuming a Gaussian-shaped electron heating profile (consisting of heating intensity and vertical extent), running the modified SAMI2 using that profile, and comparing to the ISR-derived measured temperature profile. The heating profile was then iterated until the modeled temperature was in close approximation to the ISR electron temperature results. This heating profile serves to model the bulk ohmic heating that takes place during high-power HF experiments. It was necessary to modify the SAMI2 source code to include this heating profile. Tracking code was developed which would locate the altitude at which the local electron density matches a 
parameterized density value. For this work, two different density matching schemes were investigated. Initially, the density parameter was set equal to the density value obtained by using the ionospheric plasma frequency, given by:

$f_{p}=\left(\frac{N_{e} e^{2}}{2 \pi \varepsilon_{0} m_{e}}\right)^{1 / 2}$

For the HF heating frequency used in this experiment, $3.15 \mathrm{MHz}$, the associated matching density is 1.24 electrons $/ \mathrm{cm}^{3}$. Using this value resulted in "burn through", in which the density cavity which is formed due to the ohmic heating reduces the maximum electron density value below 1.24 electrons $/ \mathrm{cm}^{3}$, leading to a cessation of heating for even modest values of energy input. This effect is also is observed in the ISR data, Figure 14, in which the local electron density at the center of the cavity is less than 1.24 electrons $/ \mathrm{cm}^{3}$. This burn through was ameliorated by matching the upper hybrid frequency of the ionospheric plasma, given by:

$f_{u h}^{2}=f_{p}^{2}+\left(\frac{e B}{2 \pi m c}\right)^{2}$

This results in a matching density of 1.14 electrons $/ \mathrm{cm}^{3}$, and removed most of the burn through occurrences. The additional heat due to the high power HF transmissions was then applied in a Gaussian shape with a half-width of $60 \mathrm{~km}$, chosen based on the ISR results.

In order to model the effect of the fast, non-thermal electrons that are generated in the nonlinear interaction of the HF wave and the plasma, changes were made to the ion-molecular charge exchange chemical reaction rates, in particular the 
$\mathrm{N}_{2}+\mathrm{O}^{+}=\mathrm{NO}^{+}+\mathrm{O}$ reaction, which depends strongly on the vibrational temperature of the nitrogen molecule. ${ }^{38}$ Higher energy electrons $(\sim 2-3 \mathrm{eV})$, such as those created by nonlinear acceleration processes associated with high power HF heating, have a significantly greater cross section to excite the vibrational modes of the nitrogen molecule. The increased vibrational temperature of the nitrogen molecules increases the cross section of the reference charge exchange reaction, resulting in an increased ratio of nitrous oxide ions to oxygen ions. Nitrous oxide ions have a recombination rate with electrons several orders of magnitude larger than oxygen ions, so increasing the vibrational temperature of the nitrogen molecules has a net result of increasing the overall electron-ion recombination rate during heating experiments. This vibrational temperature is not currently represented in the SAMI2 chemistry code, and so a alternative method has been devised. It was decided to directly modify the ionmolecular exchange rate in the affected volume during heating. This was chosen in part in order to maintain the appropriate $\mathrm{N}_{2}, \mathrm{O}^{+}, \mathrm{NO}^{+}$, and $\mathrm{O}$ concentration ratios. A parameter was introduced into the SAMI2 code which varies the ion-molecular charge exchange rate of the $\mathrm{N}_{2}+\mathrm{O}^{+}=\mathrm{NO}^{+}+\mathrm{O}$ reaction in the interaction region, using the same tracking and matching code described previously. This parameter represent the increase of the molecular nitrogen's vibrational temperature relative to it's thermal temperature. The oxygen ion charge exchange reaction rate is strongly dependent on the both the values and the ratio of these temperatures. The results, and lessons learned, of this theoretical modeling is discussed in section $\mathrm{K}$.

\footnotetext{
${ }^{38}$ Milikh, G. M., Role of chemical effects in the formation of electron concentration depletions during HF heating of the ionosphere, Journal of Atmospheric and Terrestrial Physics, 52, 119-123, 1990
} 
G. Phenomenon being Investigated: Large -Scale Artificially Generated Ionospheric Cavities

G.1.

High-power, high-frequency (HF) ionospheric modification facilities have been developed over the last thirty years and used to investigate the ionosphere by exciting a wide range of nonlinear responses in the ionospheric plasma. HF ionospheric modification experiments have been performed at facilities in Russia (Sura) (?), Norway (EISCAT) (?) and the US (Arecibo, HIPAS, HAARP) (?), using high-power HF antenna array systems. These systems have Effective Radiated Powers (ERP) ranging from just under $80 \mathrm{MW}$ to over $1200 \mathrm{MW}$. These experiments have demonstrated the capability to generate electron density cavities via resonant electron heating in the Fregion with scale lengths of tens of kilometers in the horizontal direction and hundreds of meters in the vertical direction. A generalized schematic of HF heating is shown in Figure 6. Under optimal conditions, experimenters at Arecibo have been able to create large-scale structures with estimated electron depletion levels of greater than $40 \%$. For the heating facility at Arecibo Observatory, these optimal conditions are associated with an ionosphere having a low density $\left(\mathrm{f}_{0} \mathrm{~F}_{2} \leq 5 \mathrm{MHz}\right)$ and low neutral wind speeds, and can typically be found during solar minimum.

Several processes play key roles in producing these large-scale cavities. The high-power HF wave interacts resonantly with electrons at the altitude where the local plasma frequency matches the HF frequency and raises the electron temperature from 
$800^{\circ} \mathrm{K}$ to well above $2500^{\circ} \mathrm{K} .{ }^{39,40}$ This increased temperature raises the pressure in the interaction region and consequently produces a magnetic-field-aligned flow of electrons out of the heated volume. ${ }^{41}$ Also, electrons which have been accelerated by multiple reflection point crossings ${ }^{42}$ increase the recombination rate via a two-step chemical process. These fast electrons (with effective temperatures greater than $2 \mathrm{eV}$ ), increase the vibrational temperature of molecular nitrogen. This in turn results in an increased ion-molecular exchange of nitrogen and ionic oxygen. The $\mathrm{NO}^{+}$ions then quickly undergo dissociative recombination which results in a diminished electron density. ${ }^{43}$ Additionally, the cavity formed by these processes, in a phenomenon known as self-focusing, acts as a converging lens that focuses the high-power HF wave, whereby the EM field strength is increased and all of the formation processes are enhanced. ${ }^{44}$ In addition, there are physical processes in the interaction between the high-power HF wave and the ionospheric plasma that result in small-scale structuring

\footnotetext{
${ }^{39}$ Mantas, G.P., H.C. Carlson, C.H. LaHoz, Thermal response of the F region ionosphere in artificial modification experiments by HF radio waves, $J G R, 86,561-574,1981$

${ }^{40}$ Djuth, F.T., B. Thidé, H.M. Ierkic, M.P. Sulzer, Large F region electron-temperature enhancements generated by high-power HF radio waves, Geophys. Res. Lett., 14, 953-956, 1987

${ }^{41}$ Carlson, H.C., V.B. Wickwar, and G.P. Mantas, Observations of fluxes of suprathermal electrons accelerated by HF excited instabilities, J. Atmos. Terr. Phys., 44, 1089-1100, 1982

${ }^{42}$ Grach, S.M., N.A. Mityakov, V.Yu. Trakhengerts, Electron acceleration in the prescence of parametric heating of a bounded layer of plasma, Radiophys. Quantum Electron. Engl. Transl., 27, 1096-1101, 1984

${ }^{43}$ Gennady Milikh, ibid., Journal of Atmospheric and Terrestrial Physics, 52, 119-123, 1990

44 Bernhardt, P.A., et. al., The ionospheric focused heating experiment, J. Geophys. Res., 100, 1733117345, 1995
} 
that is not discernable by the differential phase technique at UHF/VHF frequencies. ${ }^{45}$, 46

${ }^{45}$ Gurevich, A.V., T. Hagfors, H. Carlson, A.V. Lukyanov, and K.P. Zybin, Electron temperature measurements by incoherent scattering in the presence of strong small scale temperature irregularities, Phys. Lett. A, 246, 335-340, 1998

${ }^{46}$ Kelley, M.C., T.L.Arce, J. Salowey, M. Sulzer, W.T. Armstrong, M. Carter, and L.M. Duncan, Density depletions at the 10-m scale induced by the Arecibo heater, J. Geophys. Res., 100, 17367-17376, 1995 


\section{H. Description of Experiment}

In January of 1998, nine Magnavox Model 1502 receivers were deployed on the island of Puerto Rico by the Naval Research Laboratory. The TEC data from these receivers, along with data from a separate Caribbean receiver chain, maintained by the Applied Research Laboratory (University of Texas at Austin), were used to form twodimensional images of electron density via the technique of computerized tomographic inversion. The locations were chosen such that they formed three chains each consisting of three collinear sites along a separate meridian. This arrangement maximizes the likelihood of an intersection between the satellite-receiver sightlines and the HF-heated volume. The CIT receiver sites included a university, a high school, National Guard bases, Civil Defense sites, and the homes of several Arecibo Observatory (AO) researchers. Figure 7 is a table that lists these sites and provides location details. The primary criterion in choosing a site is ensuring that there are few obstructions which would interfere with $\mathrm{VHF} / \mathrm{UHF}$ satellite signal reception. In addition, all sites in a given chain should be both collinear and parallel to the ground track of the beacon satellites. This is difficult in practice, and a slight variance from colinearity for the available receiver sites must be tolerated.

Once the receivers were deployed and operational, a series of experiments were performed at the Arecibo Observatory. For each prospective satellite pass, the HF heater was turned on at full power (80 MW ERP) for a period extending from thirty minutes before the pass to just after the satellite had passed beyond the horizon. The CIT receivers collected the differential phase data (which is converted to TEC in post- 
processing) while the ISR collected high-resolution information on the vertical profile of electron density.

Figure 8 is a map of the island of Puerto Rico, and indicates the locations of each site, the location of the HF heater, and the location of the AO ISR. Note that the receiver sites are distributed across the island, and that one site is very near the HF heating facility. Also note that the HF heating facility is not co-located with the ISR, but is on the northern coast, approximately $15 \mathrm{~km}$ from the ISR. Figure 9 is an isometric view showing an idealized satellite flyover, and demonstrates the three reconstructions planes associated with the distributed receiver array. 


\section{Experimental Results}

\section{I.1. Satellite Overflight}

Figure 10 indicates the island boundary with a thin black line and indicates the Arecibo heater location by a green spot. The size of the green spot is intended to depict the size of the HF transmitter beam as estimated for $350 \mathrm{~km}$ altitude, which is the approximate altitude of the F layer of the ionosphere. Also depicted is the ground track of the satellite (asterisk.) Each of the three other tracks (diamond, plus, triangle) represents the intersection of one plane, defined by the satellite pass and a specific ground receiver, and a second plane defined by $350 \mathrm{~km}$ altitude. This intersection is the “350 km altitude track" of the satellite along the ground receiver's line of sight. The diamond (triangle) symbols identify the " $350 \mathrm{~km}$ altitude track" of the westernmost (easternmost) receiver chain. Note that the triangles intersect the middle of the green spot and the plusses intersect the edge. Since the diamonds do not intersect the green spot, the signal from the westernmost chain would be expected to be unaffected by the heating.

\section{I.2. TEC measurements}

Figure 11 shows the latitudinal profile of TEC for each of the seven receivers. Each profile is compiled for the satellite pass DOY 027, 1998, 0337 UT, and is arranged to coincide with the relative position of the receiver with respect to the silhouette of the island of Puerto Rico. Figure 12 is the same TEC data after passing through a ten second smoothing filter. This process is known as de-trending and it eliminates the Ushaped curve in the slant TEC data that is due solely to geometric factors while 
highlighting any other structure present in the data. Note that the profiles associated with the westernmost receiver chain (chain A) are relatively free of structure, i.e. approximately corresponding to a $\sec (\chi)$ relationship ( $\chi$ being the zenith angle), compared to the profiles associated with the easternmost chain (chain C). This is expected since the line of sight for chain A encounters only the unmodified ionosphere whereas the line of sight for chain $\mathrm{C}$ passes through the heated region. For the signals from the center chain (chain B), slight variations in the $\sec (\chi)$ curve results from the line of sight passing through the edge of the heated region. When these data are analyzed tomographically, it is the variations from the $\sec (\chi)$ curves that are responsible for the structure in the electron density map of the ionosphere. As pointed out in section D, the TEC data is to be used for resolving the unknown TEC offset via fitting with a Chapman model, for establishing an initial guess for the tomography algorithm using that same Chapman model, and as part of the iterative process within the algorithm. The fitted Chapman model, with the associated calculated TEC profile, is shown in Figure 13 along with the measured TEC.

\section{I.3. ISR measurements}

Figure 14 is the vertical profile of electron density measured by the Arecibo ISR. This data corresponds to the beginning of the satellite pass reconstructed in Figures 15 and 16, occurring at DOY 027, 0337 UT, 1998. That the radar beam passes through the heated volume can be seen by the reduction in electron density from 350 $\mathrm{km}$ to $410 \mathrm{~km}$ altitude that reaches a maximum reduction of approximately $50 \%$ at 380 $\mathrm{km}$ altitude. Over much of the altitude range depicted, this profile can be described by a 
Chapman model. Minor deviations from the model are visible at $150 \mathrm{~km}$ altitude and are caused by chemical (e.g., long-lived metal ions) and physical (e.g., convective instabilities) processes which are ignored by the model. The primary deviation from a Chapman profile is the modification of ionospheric electron density by the HF heater. The plasma line artifact is observed as an apparent spike in electron density at $380 \mathrm{~km}$ altitude. Recall from the discussion in section $\mathrm{E}$ that this artifact does not represent real electron density, but does provide an indication of the height of the HF-absorption. The dashed line on the plot is a Chapman-fit to the data, and serves as the unmodified ionosphere baseline. As pointed out in section E, the ISR data is to be used both for resolving the unknown TEC offset as well as establishing an initial guess for the tomography algorithm. It is not used within the iterative tomographic process.

\section{I.4 Tomographic Reconstructions}

\section{I.4.a Chapman-model based calculations}

Figure 15(a-c) shows the tomographically reconstructed electron density maps of the ionosphere above Puerto Rico using the TEC data to both resolve the unknown offset and to generate an initial Chapman-profile guess for the tomographic algorithm. Figures $15 \mathrm{a}, \mathrm{b}$, and $\mathrm{c}$ are the reconstructions for receiver chains A (westernmost), B (central) and C (easternmost) respectively. The characteristic Chapman hump in the vertical electron density profile is obvious as a horizontal band on each map. Recall that the strength of any ground-based implementation of the tomographic technique lies in resolving horizontal structure. This is particularly true when a small number of receivers are spaced over a short array baseline, as in our case. Consequently it is this 
horizontal structure, particularly near the altitude of maximum electron density, that will be the focus of this discussion. Consistent with the latitudinal asymmetry visible in the TEC data in Figure 11, Figure 15a indicates a gentle rise of about 13\% in maximum electron density from the southern (left) edge to the northern (right) edge of the map. No structuring beyond a percent or two is visible on the map. This same rise is visible in Figure 15b, but here can be noticed a localized electron density depression from 18.0 degrees latitude to 18.14 degrees latitude with a maximum density reduction of roughly $5 \%$. In figure $15 \mathrm{c}$, the localized depression extends half a degree in latitude and reduces the electron density by $15 \%$. This progressive sequence in the cavity dimensions is what you would expect looking at Figures 11 and 12 which shows that evidence of the heated region should be most apparent in Figure 15c and least apparent in Figure 15a. The vertical dimension of the cavity cannot be determined due to the limited vertical resolution. Although the measurement of the horizontal structure is significantly more precise, the relationship between this measurement and the dimensions of the HFgenerated cavity involves some uncertainty because it is unknown precisely which vertical planar cut of the spheroidal cavity is being sampled. Since Figure $15 \mathrm{c}$ shows the cavity to be at least $1 / 2$ degree and since 1 degree of latitude corresponds to $118 \mathrm{~km}$ at the altitude in question, the horizontal diameter of the cavity is at least $50 \mathrm{~km}$.

It must be recalled that each tomographic image plane is not vertical, but instead lies in the satellite path receiver site plane. This results in an altitude-longitude correlation. By mapping the longitude of the Arecibo heater to the corresponding image plane altitude, it is shown that an upper portion of the heated volume is being sampled. 
Together with the magnetic-field-aligned characteristic of the cavity, this explains the apparent southward displacement of the cavity from the heater latitude in the tomographic reconstructions.

\section{I.4.b. ISR-based reconstructions}

Figure 16(a-c) shows the tomographically reconstructed electron density maps of the ionosphere above Puerto Rico using the ISR data to both resolve the unknown offset and to generate an initial vertical-profile guess for the tomographic algorithm. Figures $16 \mathrm{a}, \mathrm{b}$, and $\mathrm{c}$ are the reconstructions for receiver chains A (westernmost), B (central) and C (easternmost) respectively. The ISR vertical profiles contain much better information than the TEC-based vertical profiles fit using the Chapman model for two reasons. First, the ISR profiles are determined more directly from measurements. Second the ISR profiles contain fine scale features ignored when using the Chapman model. By using direct measurements, the TEC offset is more accurate and by incorporating the fine-scale features into the initial guess, the final profile is more accurate, i.e. the paucity of vertical resolution in this implementation of tomographic analysis is somewhat compensated. These improvements that are attributed to using the ISR vertical profile allow the identification of fine-scale horizontal stratification on top of the Chapman-like hump in all three maps in Figure 16. As in Figure $15 \mathrm{a}, \mathrm{b}$ and c, Figure $15 \mathrm{a}, \mathrm{b}$, and c shows the gentle rise in the electron density arising from the natural asymmetry in the north-south direction observed in the TEC data. The localized depressions in Figure $15 \mathrm{~b}$ and $\mathrm{c}$ appear in Figure $16 \mathrm{~b}$ and $\mathrm{c}$ with similar dimensions. In Figure $16 \mathrm{~b}$, the depression is at $330 \mathrm{~km}$ altitude and centered at 18.2 degrees latitude 
and in Figure $16 \mathrm{c}$ the depression is at $330 \mathrm{~km}$ altitude and centered at 18 degrees latitude. As before, this structuring suggests a lower limit to the horizontal cavity diameter of $50 \mathrm{~km}$. 


\section{J. Discussion of Experimental Results}

The largest contribution to uncertainty in performing ionospheric tomographic reconstructions lies with the TEC data itself. In the ideal case, coverage of all possible ray paths are complete and continuous and the reconstruction is unique. The curvature of the Earth prevents the acquisition of data at look angles corresponding to a horizontal integration path at low altitude. The limitation of having a finite number of receivers means two things. First, the coverage of ionosphere at any one moment during the pass is limited to one line of sight for each receiver. Thus any time evolution of the structure introduces artifacts into the finalized image. Second, the deviation in the line-averaged electron density due to horizontally localized structure is a greater percentage of the TEC when the receiver is directly below the structure. At off-vertical angles, the TEC includes proportionally more non-structured ionosphere compared to structured ionosphere. In addition, the TEC value obtained by the differential Doppler method is relative TEC, and requires an external determination of the offset necessary to obtain absolute TEC. Instrument-based limitations of the phase resolution correspond to a minimal detectable TEC variation, which in this study is one part in $10^{5}$. This limitation in detecting small changes in line integrated electron density prevents the detections of structures with vertical extent that is small, altitude that is far from the F-layer peak, and electron density deviation that is tiny. The tomographic method itself results in exact, unique solutions when the input data satisfies, or nearly satisfies, the ideal conditions.

A number of differences between this study and a recent study performed at the EISCAT facility can be described in terms of the constraints mentioned above. Both 
studies use comparable numbers of receivers per chain ( 3 receivers per chain for the Arecibo study, and 5 receivers in a single chain for the EISCAT study), so the temporal resolution of structure evolution would likewise be comparable. However, the EISCAT receiver array was roughly $480 \mathrm{~km}$ in latitudinal extent while our receiver array extended less than $50 \mathrm{~km}$. This configuration means that the EISCAT study had greater vertical resolution due to the long baseline for the receiver chain, while our study had greater horizontal resolution from the close spacing of receivers. Obviously, with a longer baseline for the receiver chain, the EISCAT study imaged a greater latitudinal extent. This is desirable due to the nature of both the highly variable ionosphere at high latitudes and the intense ionospheric modifications produced by the EISCAT HF heating facility.

A brief comparison of the EISCAT and the Arecibo Observatory heating facilities is in order. The EISCAT facility is located near Tromsø, Norway at the geographic coordinates $69.6^{\circ} \mathrm{N}$ and $19.2^{\circ} \mathrm{E}$. The magnetic dip angle at that location is approximately $78^{\circ}$. The heating facility consists of twelve transmitters capable of generating a total $\mathrm{CW}$ power of $1.2 \mathrm{MW}$ with a frequency range of 3.85-8.0 MHz, and an antenna array gain of $24 \mathrm{dBi}$. This results in a maximum effective radiative power, after losses, of $300 \mathrm{MW}$. The Arecibo heating facility is located near the town of Islote, on the north coast of Puerto Rico at geographic coordinates $18.4^{\circ} \mathrm{N}$ and $-66.6^{\circ} \mathrm{E}$. The magnetic dip angle is approximately $50^{\circ}$. The heating facility consists of 4 transmitters capable of generating a total $\mathrm{CW}$ power of $800 \mathrm{~kW}$ with a frequency range of 3.175-8.0 $\mathrm{MHz}$, and an antenna array gain of $23 \mathrm{dBi}$. This results in a maximum effective 
radiated power, after losses, of $80 \mathrm{MW}$. The EISCAT facility can therefore inject a significantly greater level of radio-wave energy into the ionosphere.

The work performed here has a number of possibilities for extension. Recently developed differential Doppler receiver systems are compatible with a greater number of beacon frequencies, significantly increasing the fraction of currently orbiting beacon satellites available for CIT studies. By expanding the number of satellites involved in each study, the data can be accumulated in a shorter period of time. Therefore, the high-power HF heating research community will have more opportunities to take tomographic snapshots of phenomena associated with an artificially perturbed ionosphere and the ionospheric research community as a whole will have more frequent monitoring of the behavior of the ionosphere. A number of new beacon satellites have recently been launched, with plans for even more in the near future. Also, over the next few years it is anticipated that a number of low earth orbiting satellites will be launched that include a differential Doppler receiver as part of their payload. Receiverequipped satellites will be able to provide horizontal integration paths which are currently unavailable. As discussed in the CIT section, horizontal integration paths contribute important information in the reconstruction algorithm. 


\section{K. Discussion of Theoretical Results}

As discussed in Section F, a two stage approach was used in modeling the effects of the high power HF heater on the ionosphere. First, the ohmic heating caused by the interaction of the radio wave and the ionospheric plasma is simulated by directly introducing heat to the electrons in the region of HF absorption where the local plasma frequency is near the frequency of the impinging radio wave. Secondly, a portion of the effects of the fast, non-thermal electrons generated in the nonlinear interaction of the HF wave and the ionosphere are modeled through incorporating a simulated increase in the nitrogen vibrational energy by modifying the ion-molecular charge exchange chemical reaction rate for the reaction $\mathrm{N}_{2}+\mathrm{O}^{+}=\mathrm{NO}^{+}+\mathrm{O}$. The location of the heated interaction region is dynamically set during each time step of the simulation to the altitude at which the local upper hybrid frequency equals the HF heater frequency specified in a parameter file.

The first step was to determine the SAMI2 input parameters which would result in an ionosphere which was as similar as possible to the observations made the night the TEC measurement occurred. A normal SAMI2 modeling run involves defining the input parameters which are used by the code to begin and then evolve the model. Typically, the model is allows to run for twenty four simulated hours, and then the results are output to data files. The parameters used in SAMI2 include the day, year, $10.7 \mathrm{~cm}$ solar flux, the 3-day average of the $10.7 \mathrm{~cm}$ solar flux, and the geomagnetic disturbance index $A_{p}$. Initially, entering values for these parameters which matched those for the night of the experiments resulted in unusual SAMI2 results. Below a 
certain value of the $10.7 \mathrm{~cm}$ solar flux, no ionosphere would develop. Consultation with the SAMI2 code developer, Dr. Joe Huba, led to the discovery that there was an error in a third-party code used within SAMI2 to derive the a version of the Chapman function which in turn drove the electron photoproduction subroutines. A new version of SAMI2 was released, in part due to this discovery, which fixed this error and made a few other changes to the code. This new code, when used with the parameter values from 27 January 1998, provided an ionosphere which nearly matched observations. However, the ion temperature was greater than that measured by the ISR (shown in Figure 17), and so the background neutral temperature was lowered using a parameter provided for that purpose. The undisturbed ions are in thermal equilibrium with the background neutrals, and so the ion temperature was reduced as well. The nominal SAMI2 model run now matched the observations.

The ohmic heating profile was chosen by matching the results of a SAMI2 simulation run with the ISR electron temperature data shown in Figure 17. After a number of simulation runs, it became apparent that for even moderate values of heating input, heating efficiency was actually reduced as the heating input increased. The source code was modified to provide step-by-step diagnostic output with respect to the various electron heating parameters, and it was discovered that a numerical instability was occurring during the artificial heating cycle. Decreasing the time step reduced the magnitude of the instability, and it was determined that a time step of one half second during the artificial heating interval eliminated the instability. For comparison, the SAMI2 nominal time step is on the order of ten seconds. Software code was introduced 
to reduce the time step to one half second solely during the heating interval, in order to prevent on undue increase in the time for a model run. Consultation with Dr. Huba after implementing the change indicated that this was the correct approach.

After a number of simulation runs using this final SAMI2 code, a heating scale height of 60 kilometers and a heating intensity of $941 \mathrm{~K} / \mathrm{s}$ applied to the electrons were chosen as the best fit to the temperature profile data. The charge exchange reaction rate was unmodified during these simulation runs. The results of this SAMI2 simulation are shown in Figures 18 through 22. Figure 18 is a time/altitude plot of the electron temperature above the Arecibo Observatory for a representative, non-heating run. The time is in UT, and the model was run for twenty four simulated hours prior to the displayed data in order to eliminate any early variances in the model. Figure 19 is the result of a simulation run, with the heater-on period occurring for twelve minutes, starting at 0300 hours. A twelve minute heating time was chosen due to limitations inherent in the two-dimensional constraints of SAMI2. Since we are limited to heating along a specific flux tube, non-realistic consequences occur when simulating extended heating periods. Figure 20 shows the difference in temperature between the nominal background ionosphere in Figure 18 and the heated ionosphere in Figure 19. Figures 19 and 20 demonstrate the match to the increase in electron temperature as obtained via the ISR as shown in Figure 17. Figure 21 is the nominal electron density background result using the 27 Jan 1998 ionospheric parameters. Figure 22 is the electron density during a heating simulation run at $941 \mathrm{Ks} /$, and Figure 23 shows the change in electron density due to heating, as compared to the background ionosphere shown in Figure 21. 
The reduction in electron density of $-5.08 \mathrm{e} 4$ electrons $/ \mathrm{cm}^{3}$ is less than the 5.65e4 that is observed in the temperature corrected ISR data. The difference is accounted for by recalling that the SAMI2 heating method models only the bulk, ohmic heating of the electrons due to their interaction with the high power HF electromagnetic field. The suprathermal tail of the modified electron distribution function, as described in Section $\mathrm{F}$, is has been modeled by modifying the charge exchange rate associated with the $\mathrm{N}_{2}+\mathrm{O}^{+}=\mathrm{NO}^{+}+\mathrm{O}$ reaction. Figure 24 is a plot of the maximum reduction in electron density when both the ohmic heating and the reaction rate are included, shown as a function of the rate modification factor (i.e., a modification factor of two indicates an increase in the reaction rate by a factor of two). The curve indicates that a value of the modification factor 2.02 is required to match the observed density reduction of $5.65 \mathrm{e} 4 \mathrm{electrons} / \mathrm{cm}^{3}$. A vibrational / translation temperature differential of 308 degrees $\mathrm{K}$ corresponds to the reaction rate factor of 2.02 , for the translational temperatures observed during the experiments. This is in good agreement with previous theoretical heating efforts addressing the effect of the vibrational states of nitrogen on HF heating experiments ${ }^{47}$. The final modeling result for the electron density is shown in Figure 25, including both the ohmic heating and the chemistry effects. Figure 26 illustrates the difference between the pure ohmic heating and that which includes the chemistry modifications. The chemical effects increase the density reduction rate, and slow down the effective electron replacement rate.

\footnotetext{
${ }^{47}$ Vlasov, M. N., Kelley, M. C., Gerken, E., Impact of vibrational excitation on ionospheric parameters and artificial airglow during HF heating in the F region, J. Geophys. Res., 109, A09304, doi:10.1029/2003JA010316, 2004
} 


\section{Conclusion}

It has been shown that the CIT technique can be applied to the threedimensional, high-resolution imaging of artificially generated ionospheric cavities above the HF heater at Arecibo Observatory. Current geometric constraints necessitate the use of supplementary data to determine the vertical profile of electron density. CIT is used to resolve the density/temperature ambiguity in ISR measurements. The differential phase technique measures only the electron density of the ionosphere. The ISR backscatter signal, as indicated in section $\mathrm{E}$, is dependent on both the electron density and the electron/ion temperature ratio. Self-consistent analysis methods can be used to determine the temperature ratio, but they are not purely empirical and require assumptions to be made about the ionosphere. By combining the TEC data with the ISR data, the effects of the temperature ambiguity are minimized.

The experimental observations were supported by theoretical work using the open-source SAMI2 ionospheric model. Methods were developed to model both the ohmic heating of the electrons and the suprathermal heating caused by nonlinear effects. Modeled ohmic heating values of $941 \mathrm{~K} / \mathrm{s}$ match the observed heated temperature profiles, and an increase of the $\mathrm{N}_{2}+\mathrm{O}^{+}=\mathrm{NO}^{+}+\mathrm{O}$ reaction rate by a factor of 2.02 results in a matched electron density reduction of $5.65 \mathrm{e} 4$ electrons $/ \mathrm{cm}^{3}$. 
Figure 1a: Chapman theory: Geometry

\section{Sun \\ 謧}

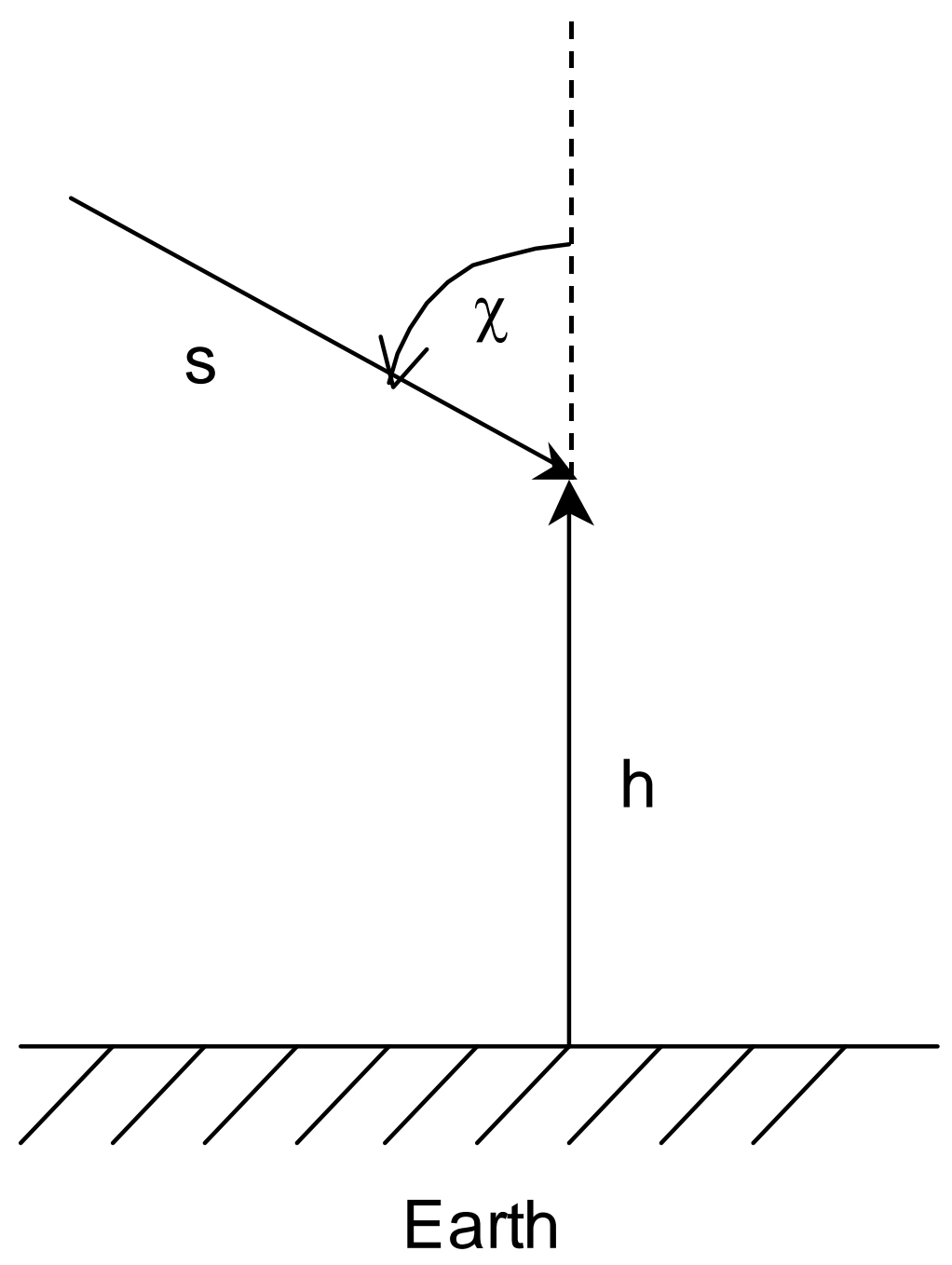


Figure 1b: Chapman theory: Hydrostatic gas and Intensity Profile

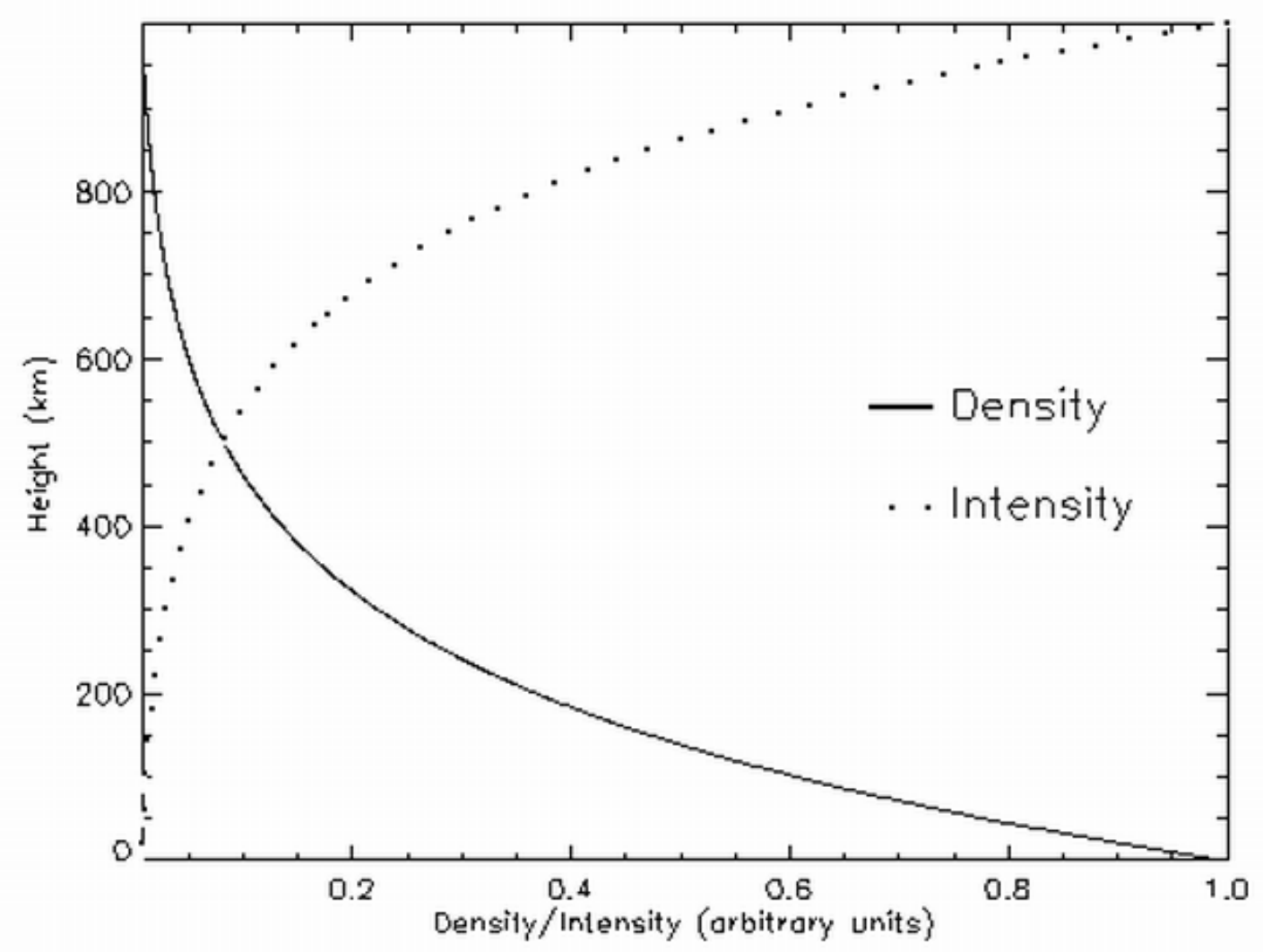


Figure 2: Chapman production example

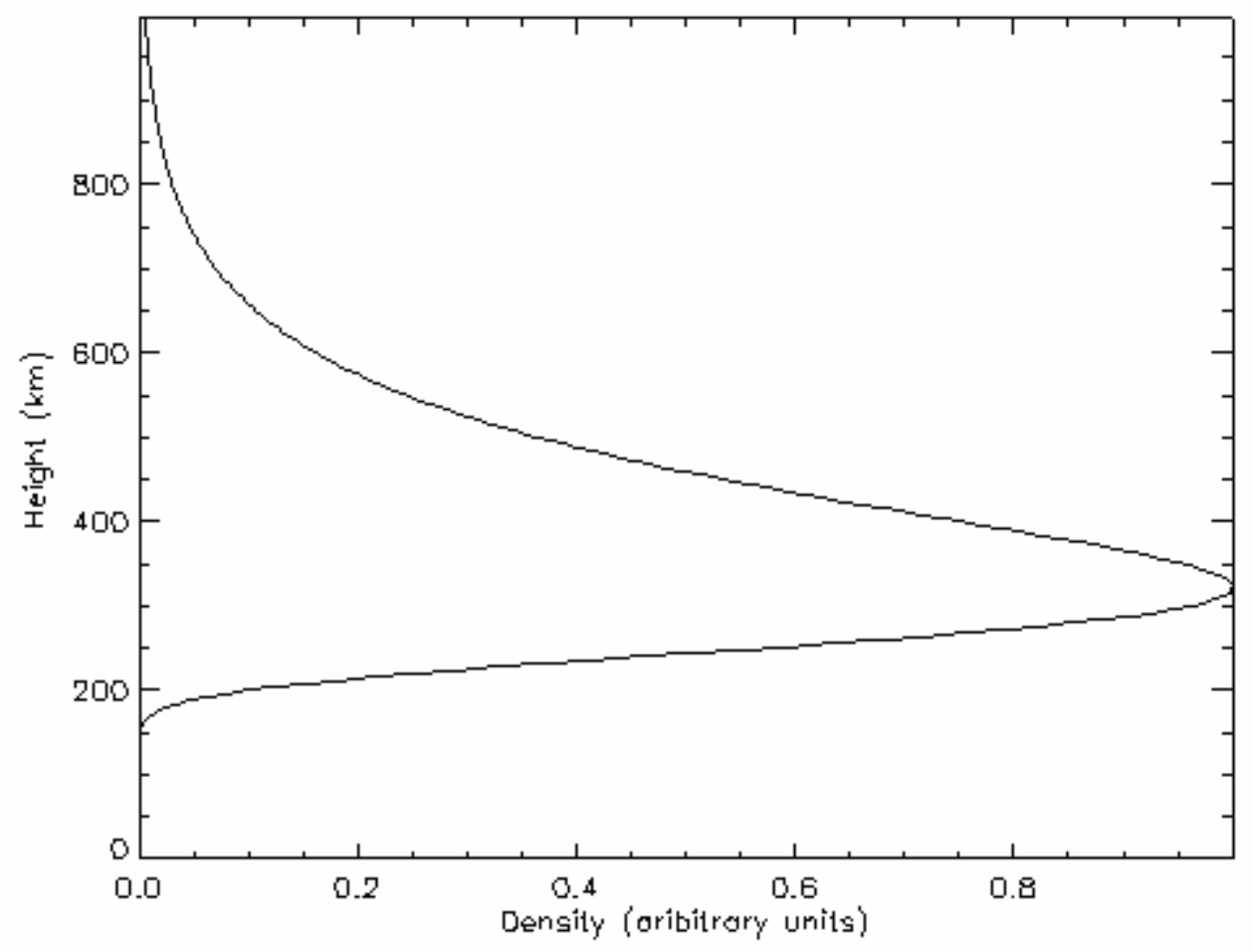


Figure 3a: 1/e Intensity Height

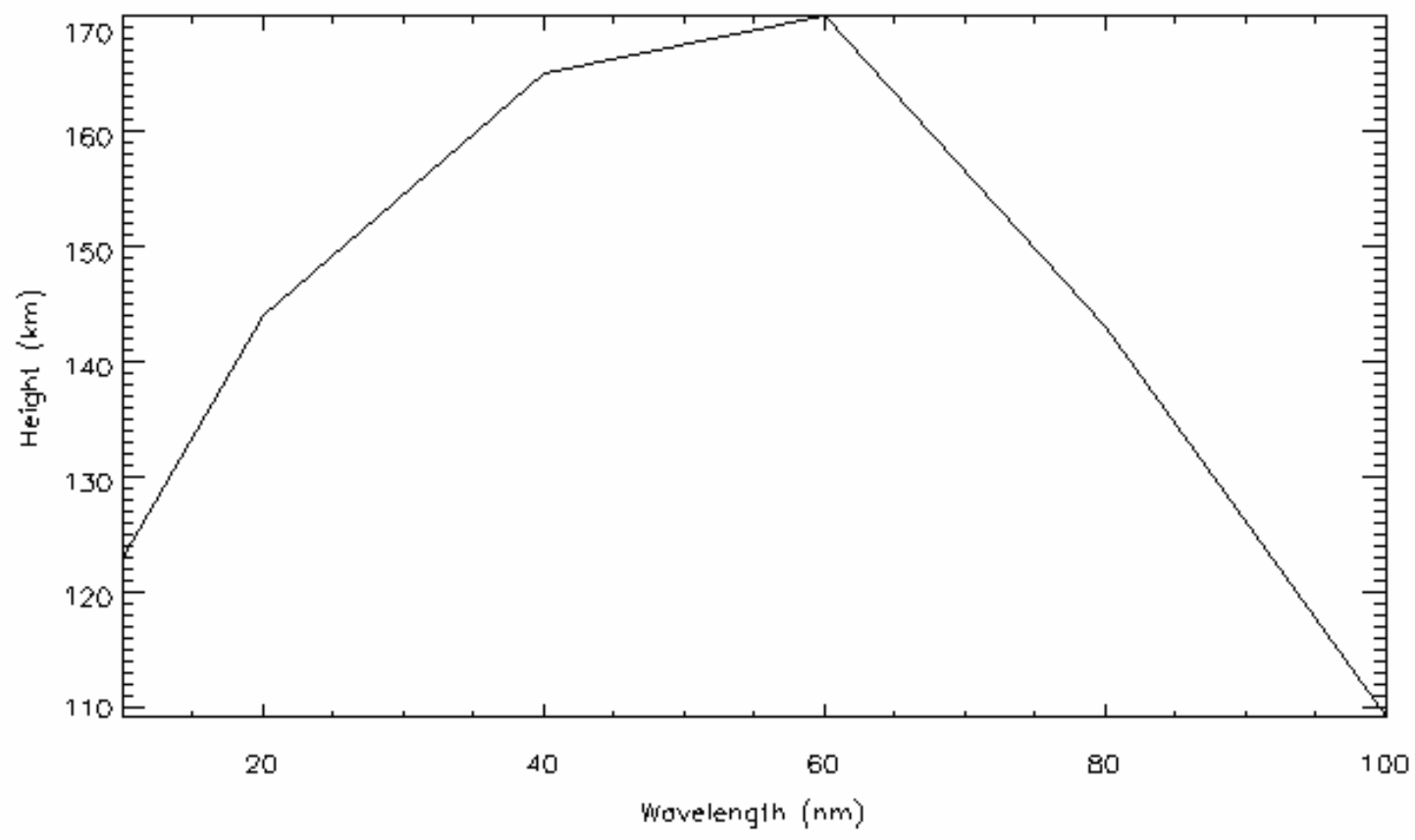


Figure 3b: Multi-spectrum Chapman Examples

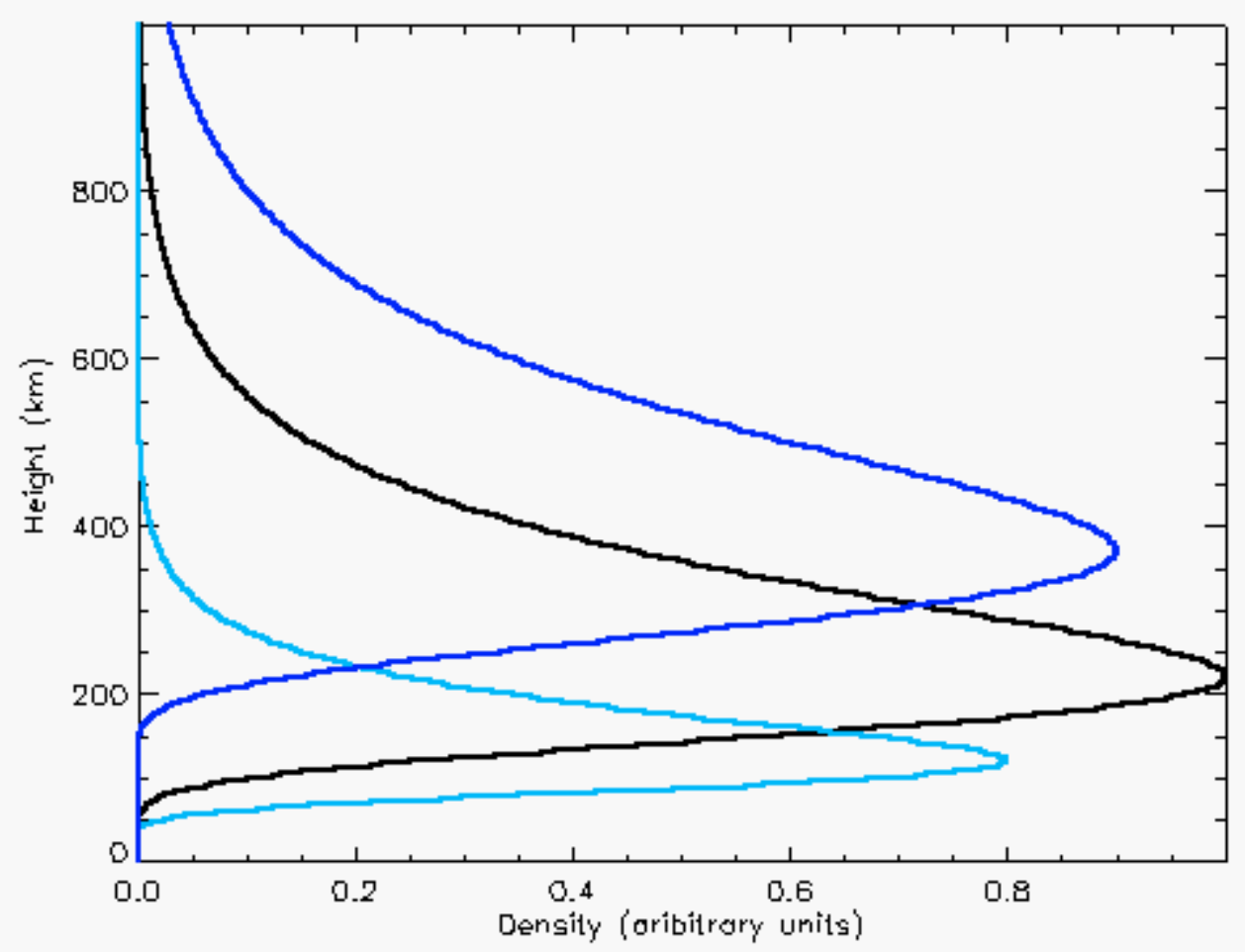


Figure 4: Equilibrium profile

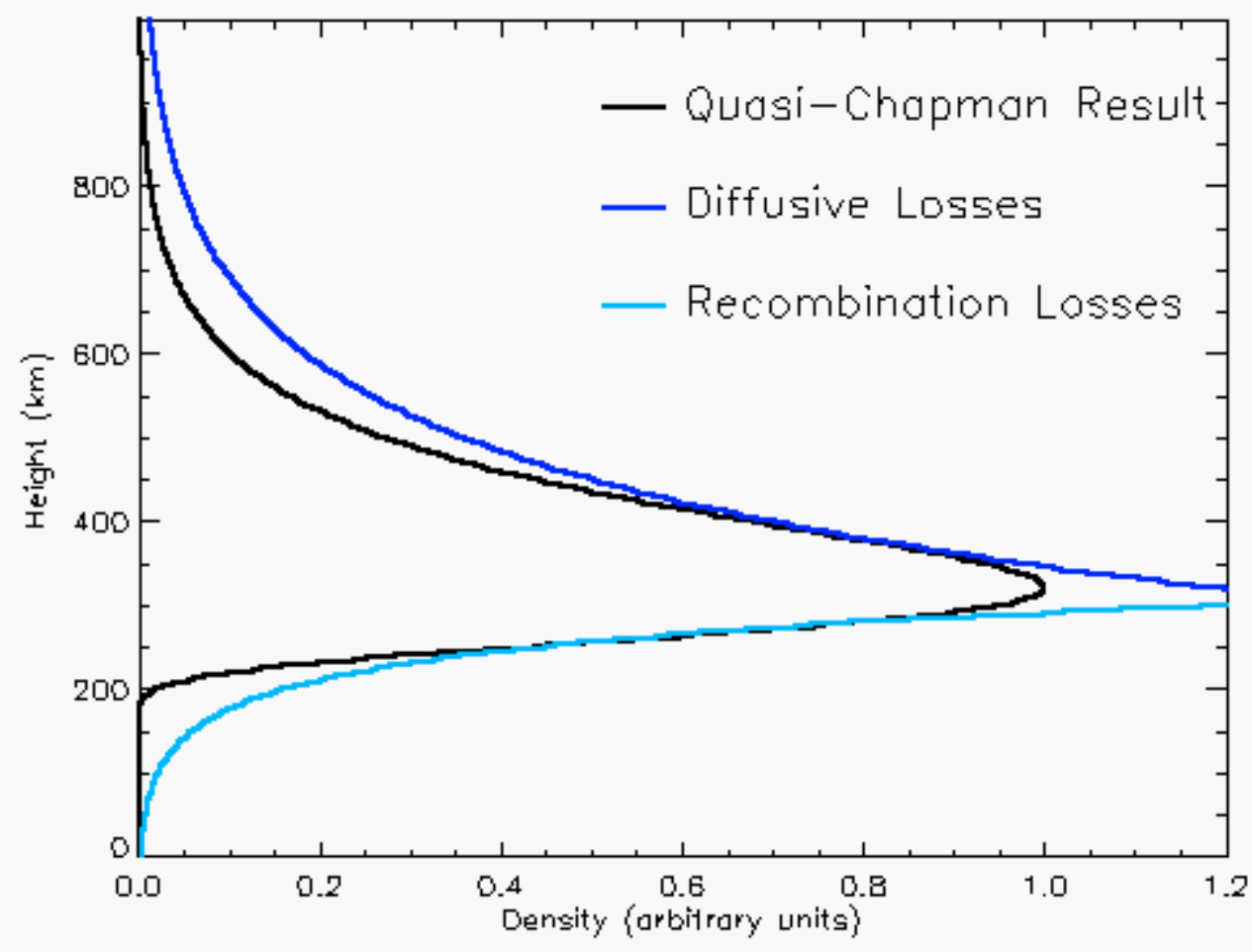


Figure 5: General CIT Observing Schematic

\section{CIT observing scheme}

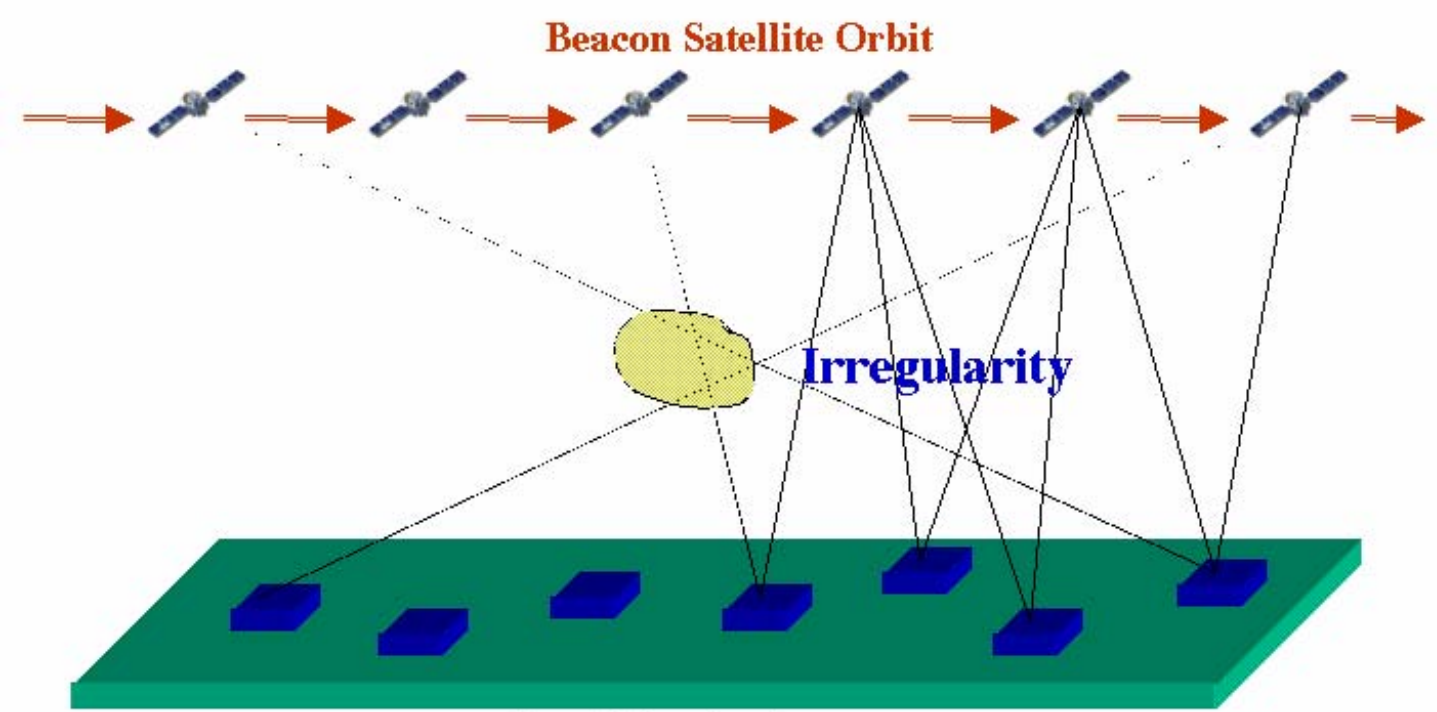

Receiving Stations 
Figure 6: HF Ionospheric Modification Schematic

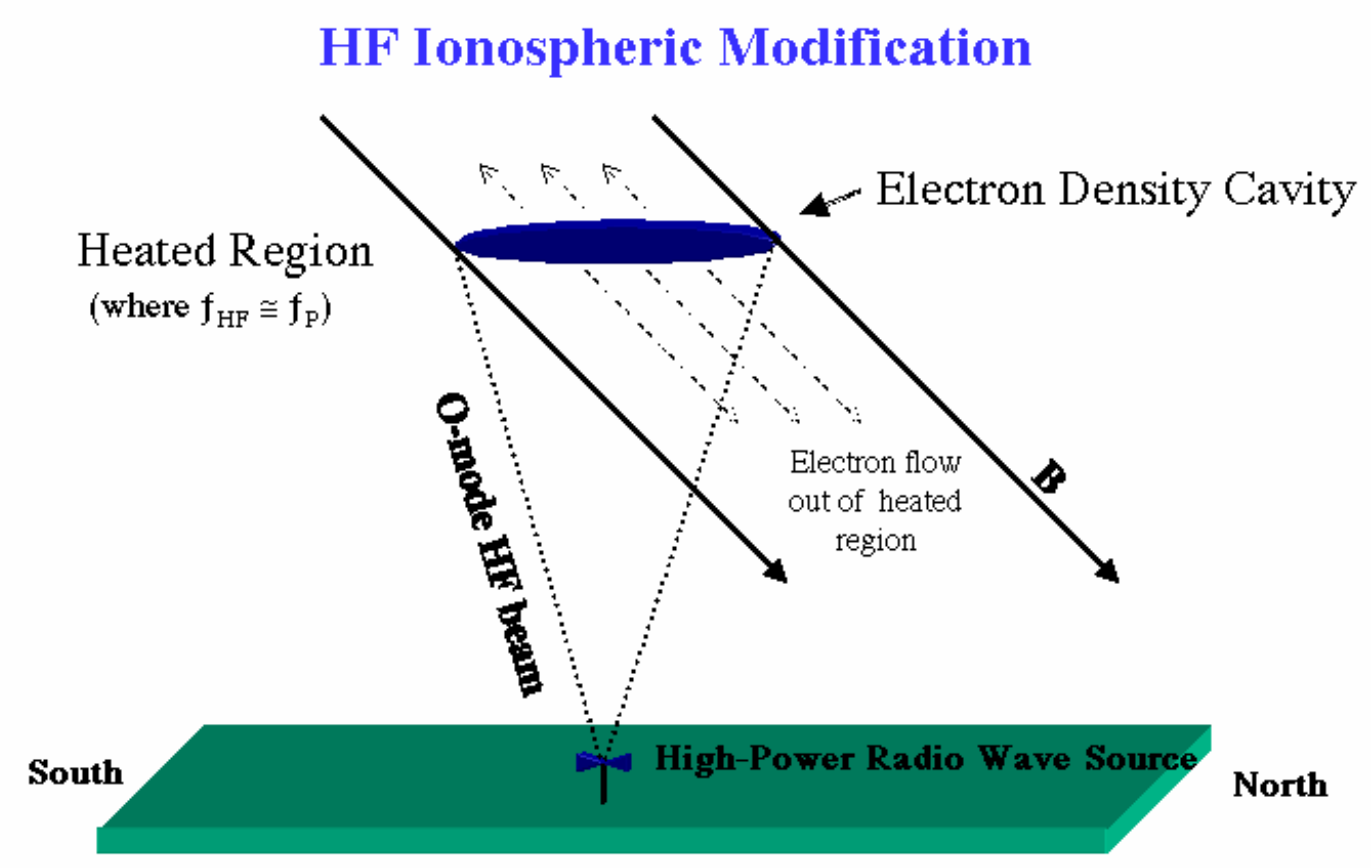


Figure 7: Table of site locations

\begin{tabular}{|c|c|c|c|c|}
\hline Site & Site ID & Latitude & Longitude & Chain \\
\hline Ramey & 2 & 18.39 & -67.13 & 1 \\
\hline Mayaguez & 10 & 18.09 & -67.14 & 1 \\
\hline Cabo Rojo & 9 & 17.96 & -67.15 & 1 \\
\hline Islote & 5 & 18.37 & -66.64 & 2 \\
\hline Utuado & 6 & 18.15 & -66.71 & 2 \\
\hline Peñuelas & 8 & 17.94 & -66.72 & 2 \\
\hline Sabana Seca & 53 & 18.38 & -66.17 & 3 \\
\hline Cidra & 1 & 18.06 & -66.16 & 3 \\
\hline Guayama & 7 & 17.86 & -66.11 & 3 \\
\hline Bermuda & 55 & & & 4 \\
\hline
\end{tabular}


Figure 8: Arecibo 98 Receiver Deployment

\section{Arecibo 1998 CIT Receiver Deployment}

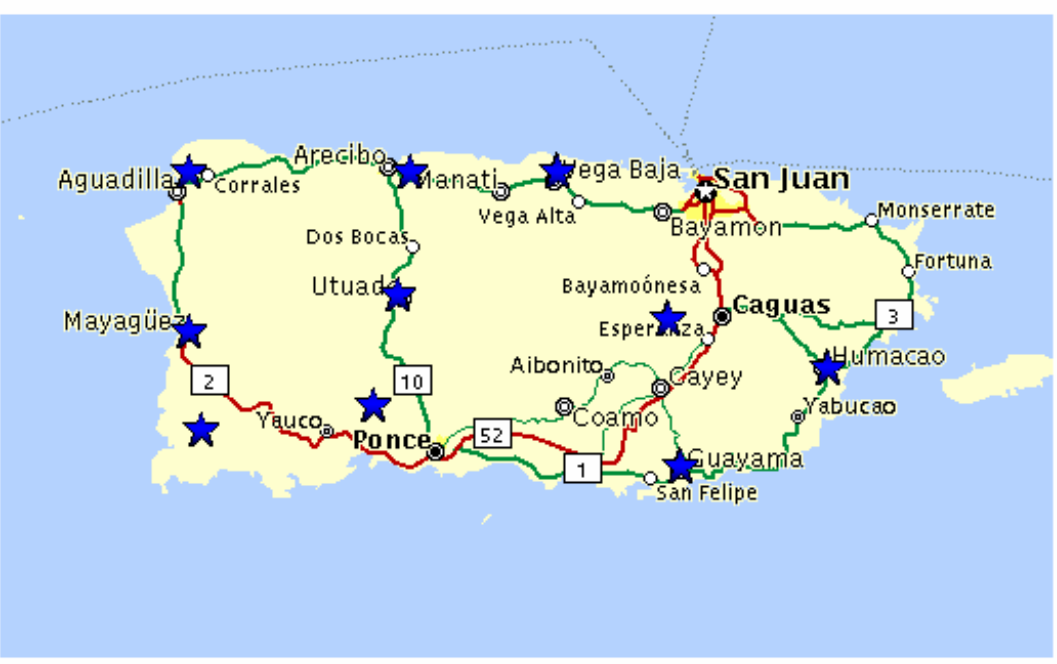


Figure 9: Idealized Observing Schematic

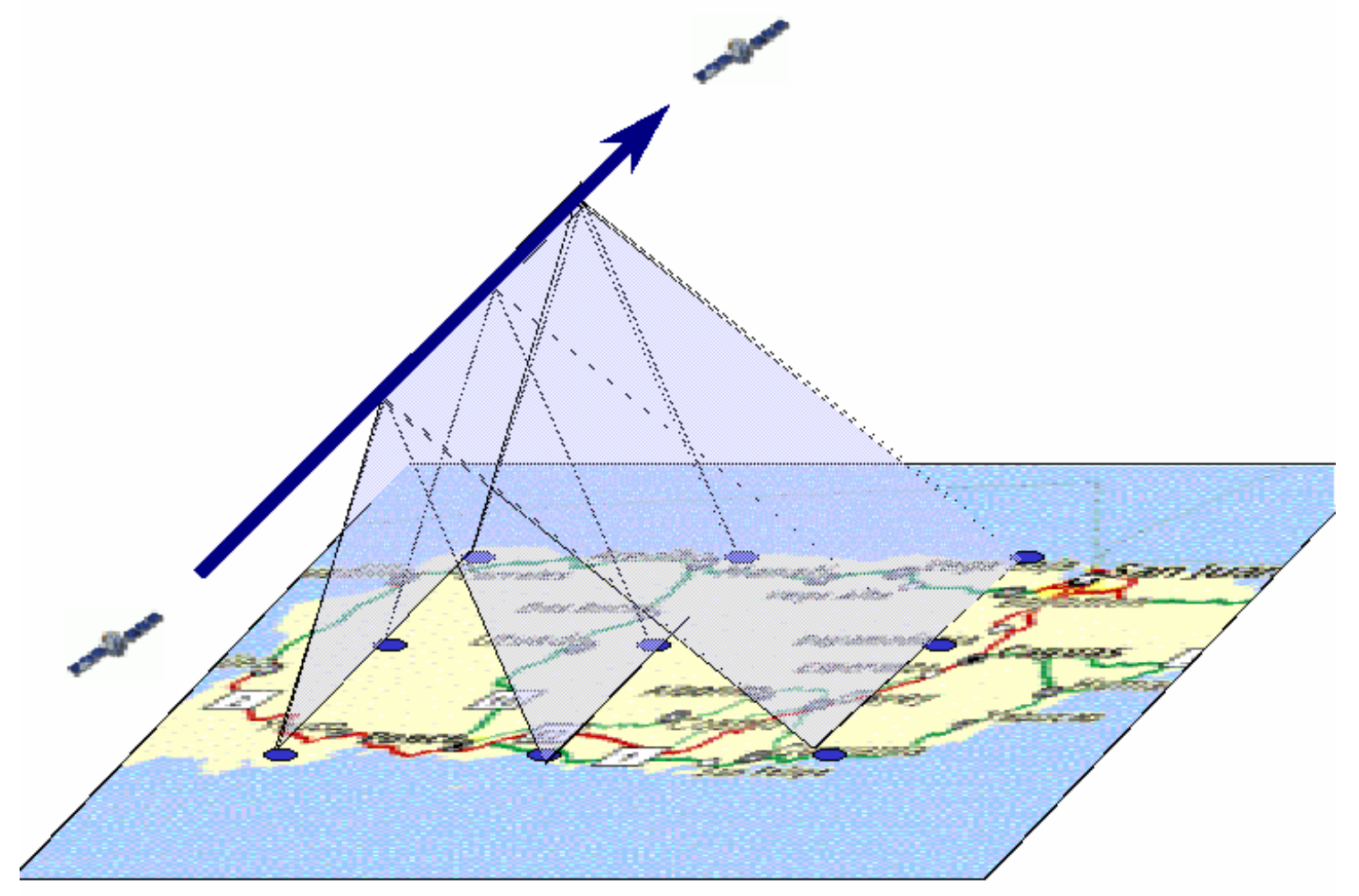


Figure 10: Satellite Overflight

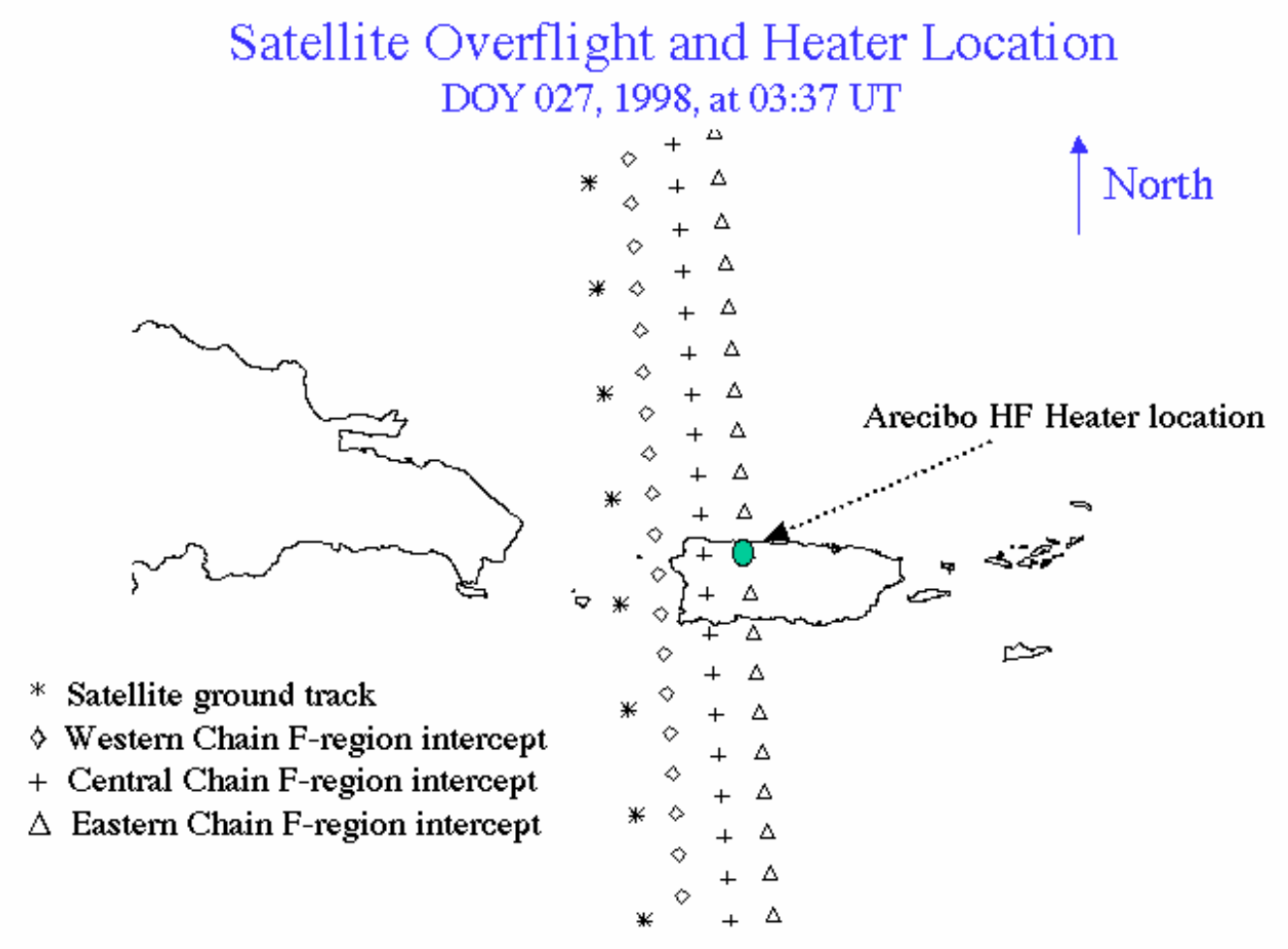


Figure 11: Slant TEC

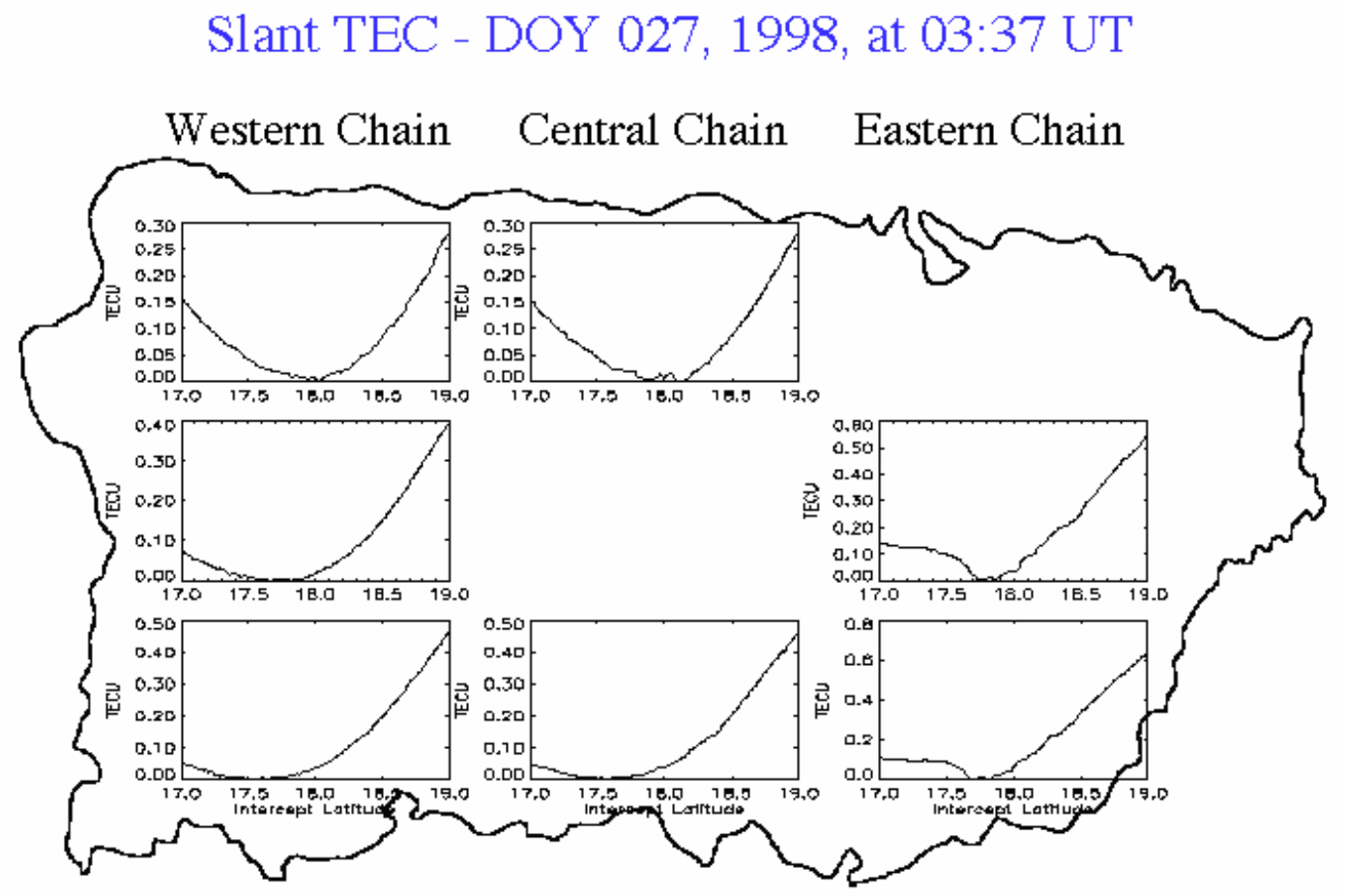


Figure 12: Detrended TEC

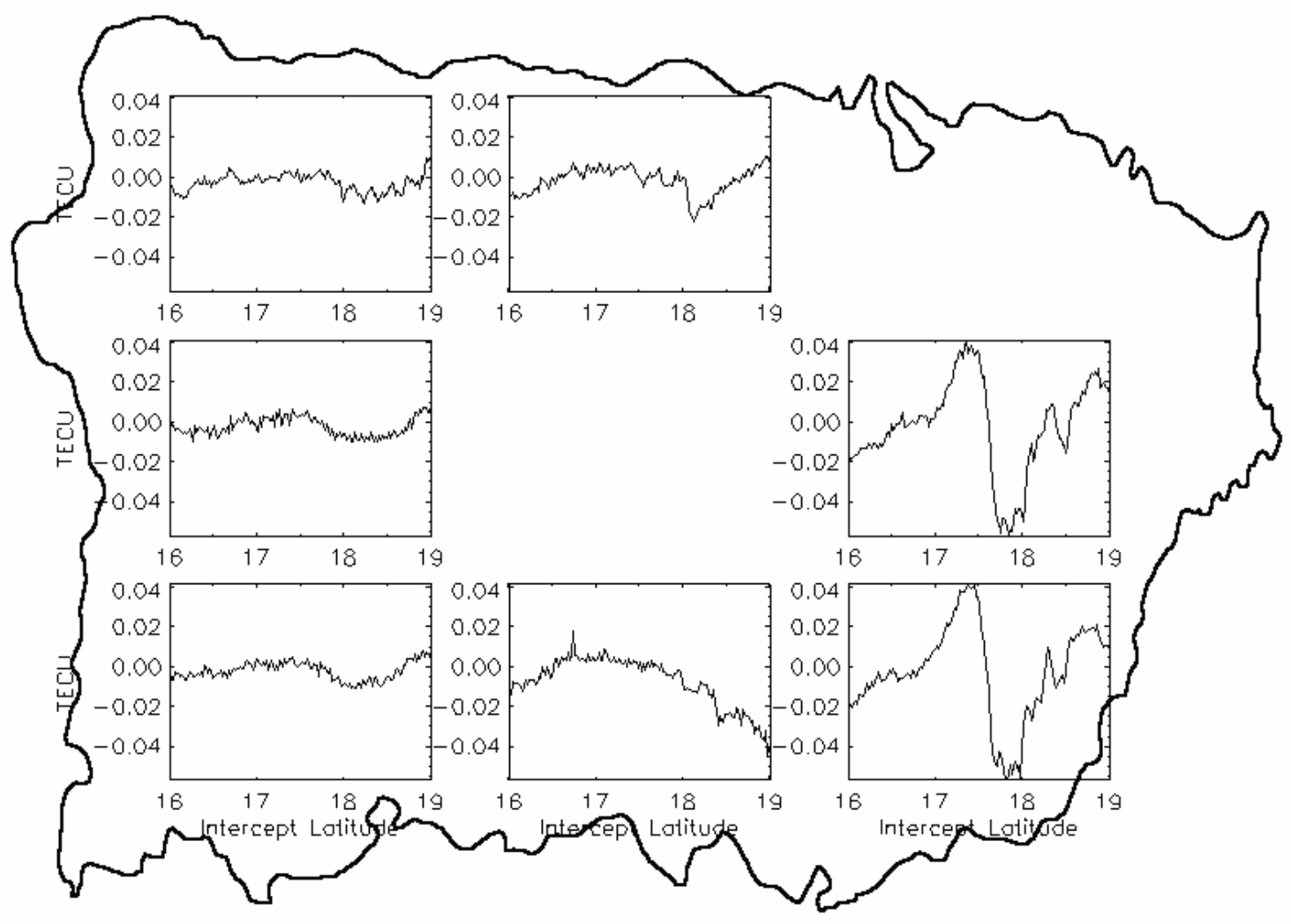


Figure 13: Chapman Model and Fit

Five-Parameter Chapman Model and Fit
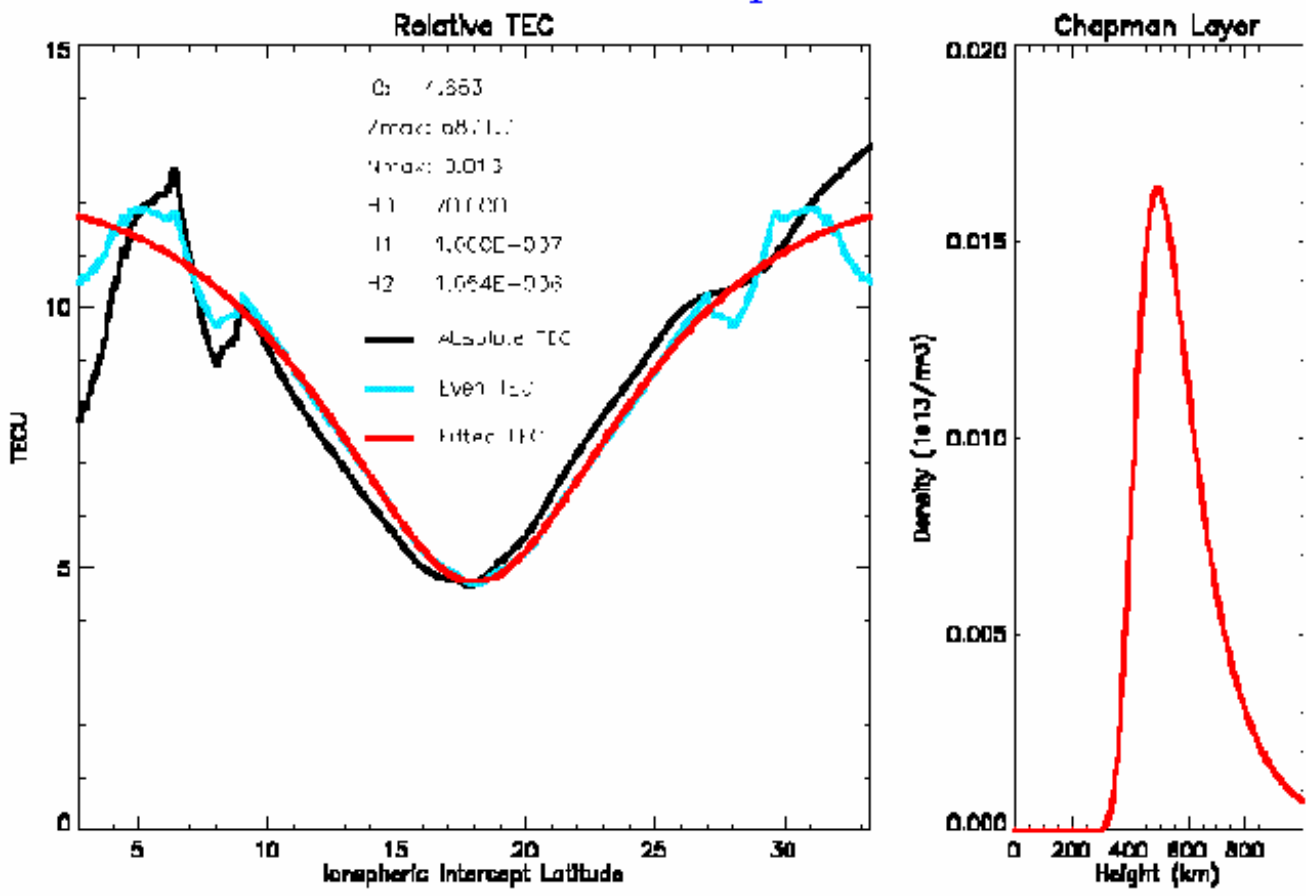
Figure 14: ISR Radar Data

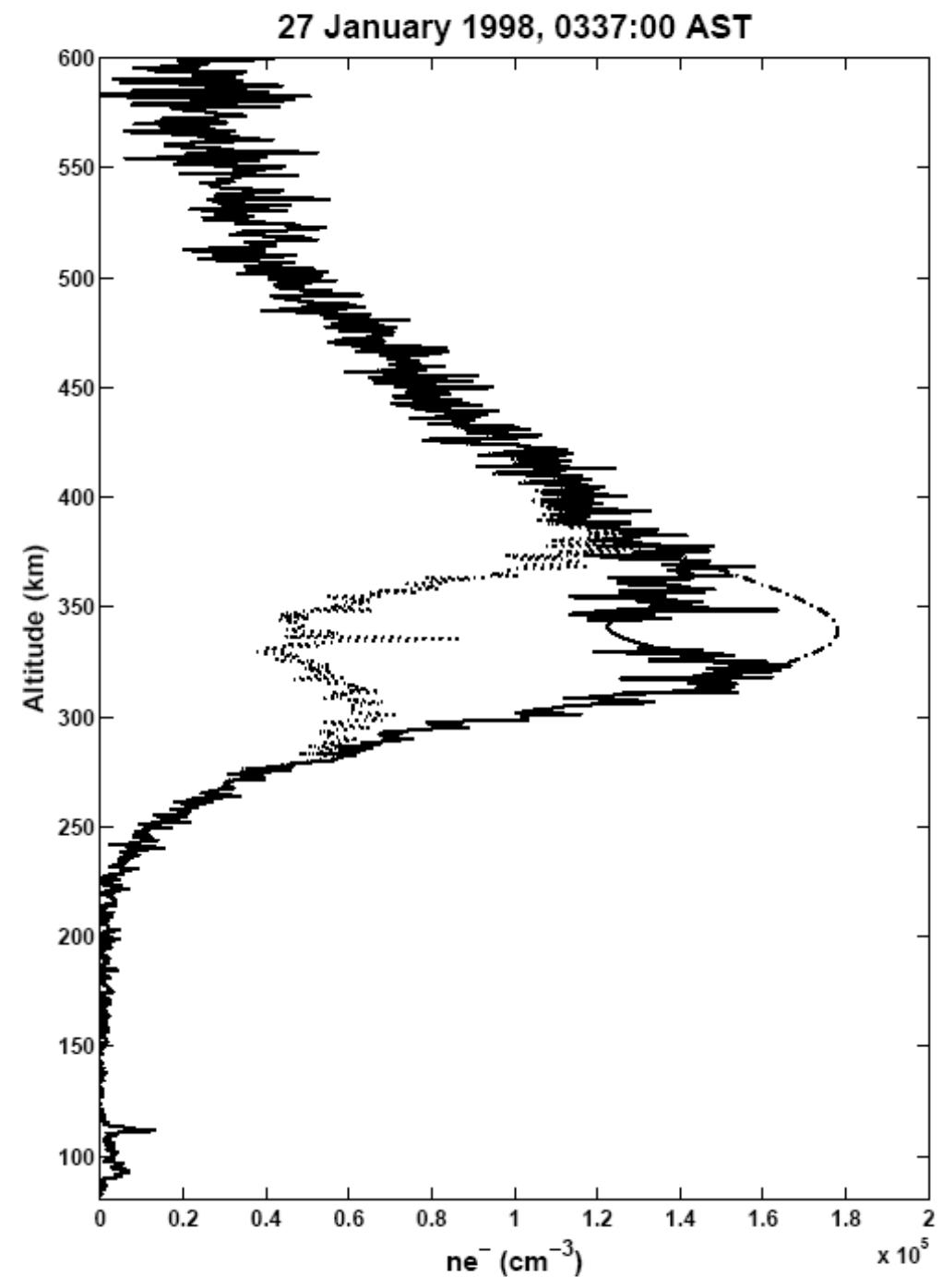


Figure 15a: Western Chain Reconstruction, Chapman Initialization
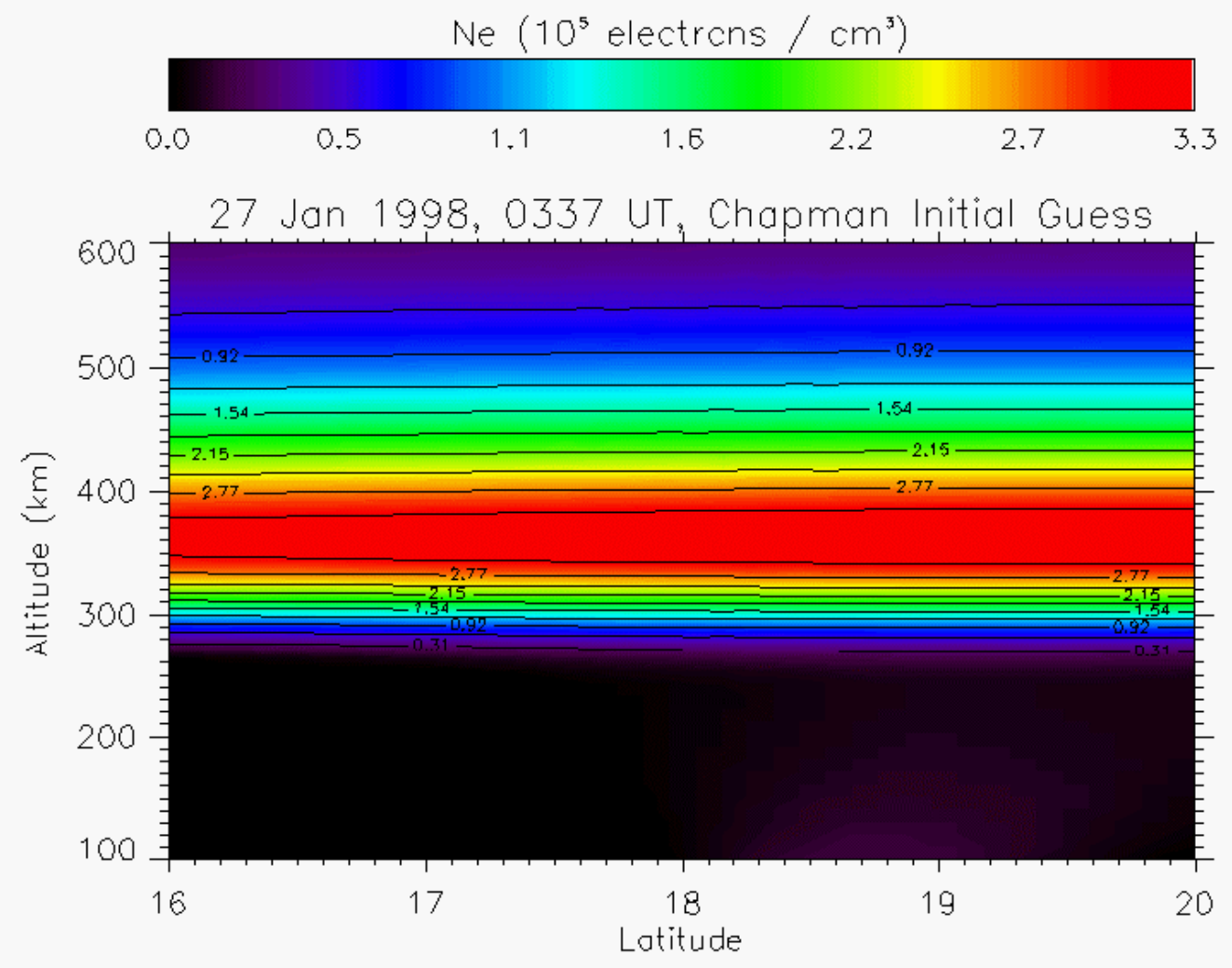
Figure 15b: Central Chain Reconstruction, Chapman Initialization
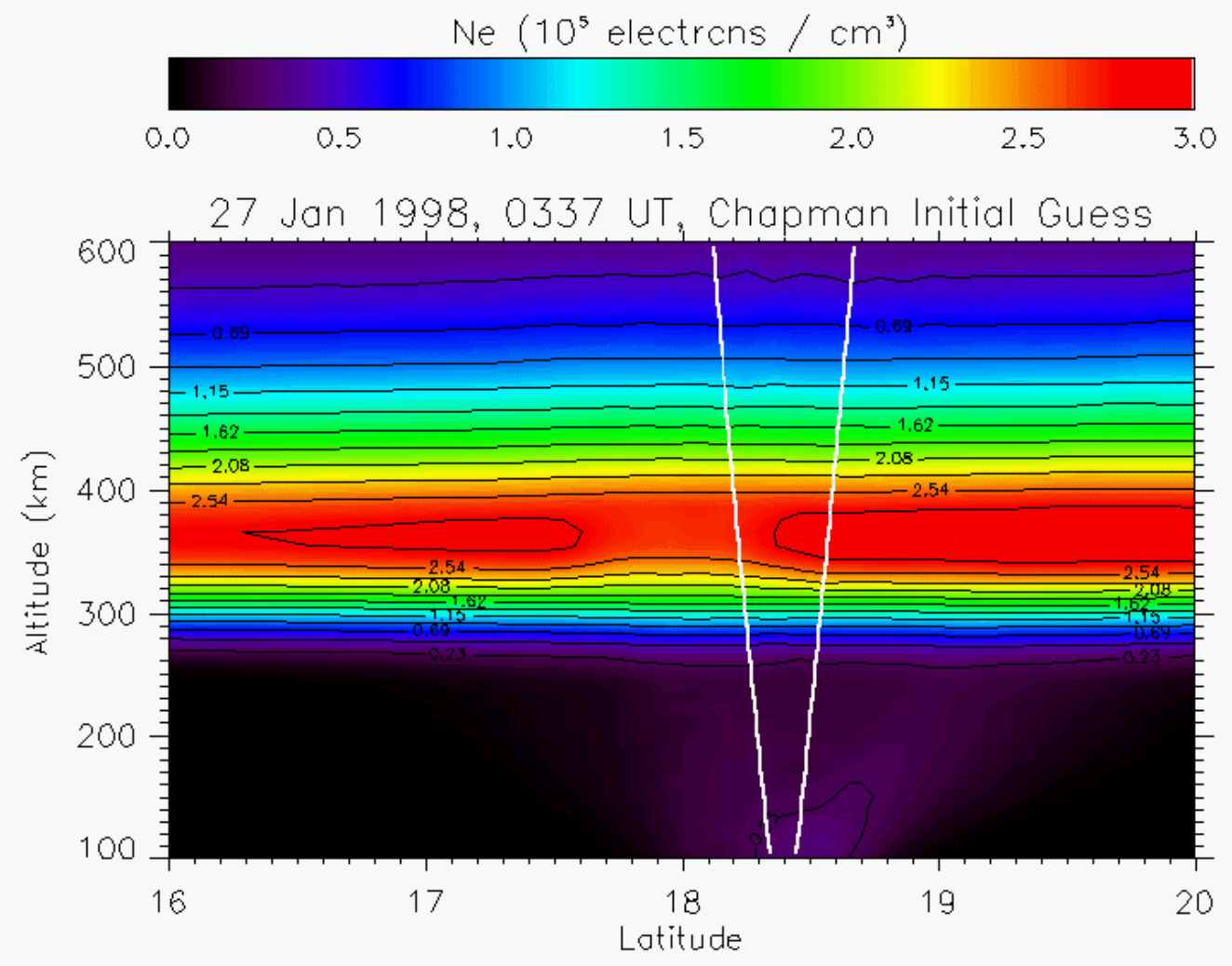
Figure 15c: Eastern Chain Reconstruction, Chapman Initialization

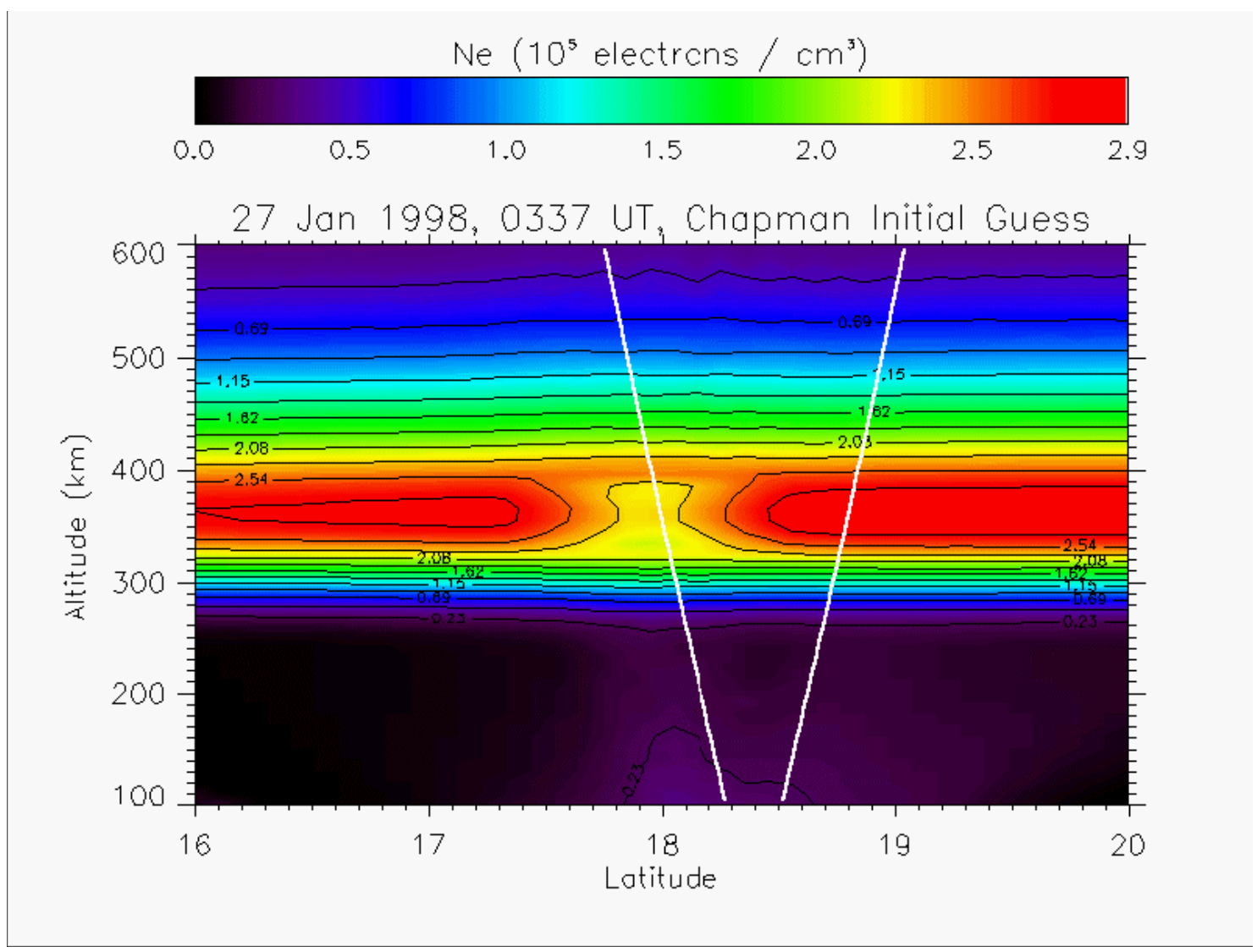


Figure 16a: Western Chain Reconstruction, ISR Initialization

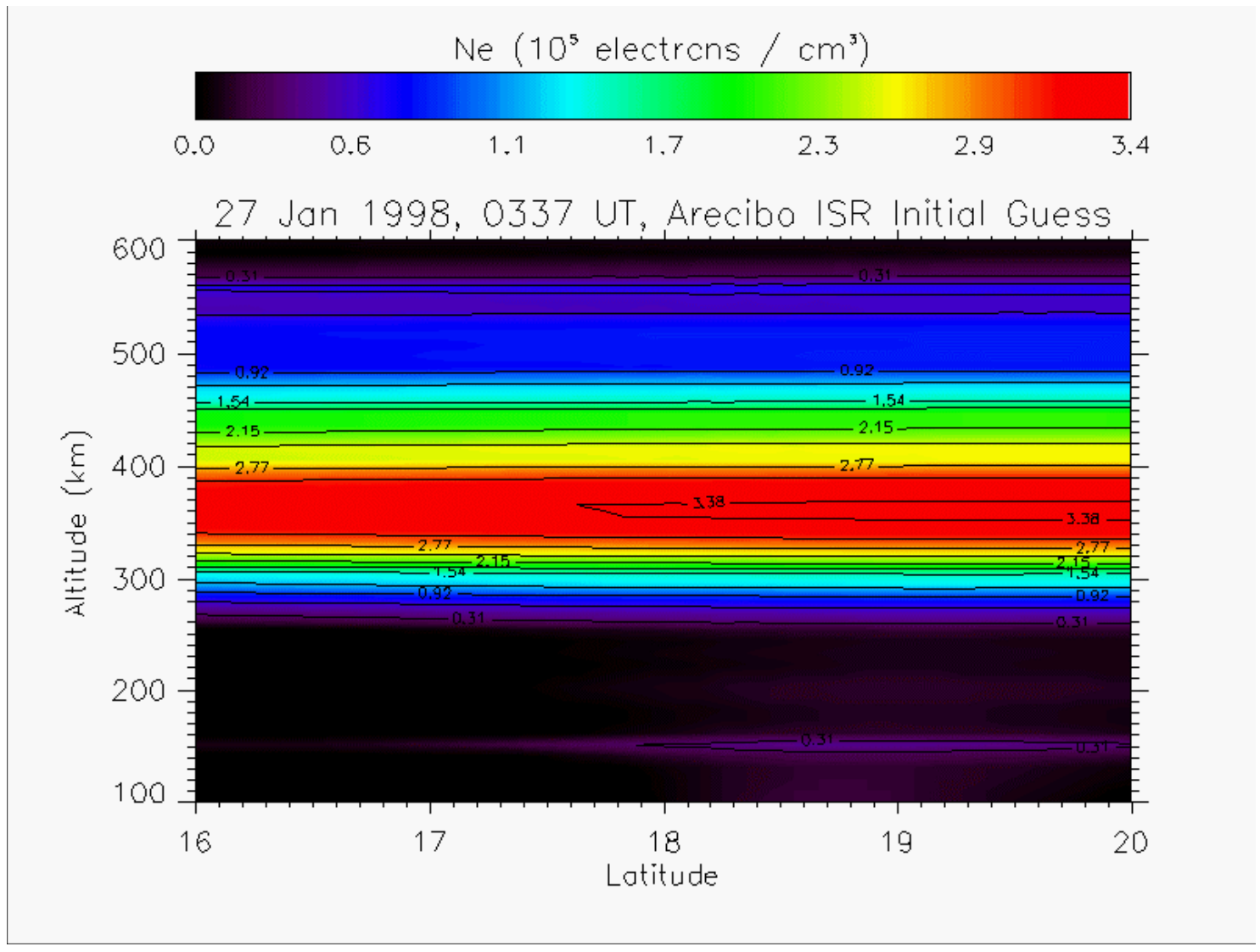


Figure 16b: Central Chain Reconstruction, ISR Initialization

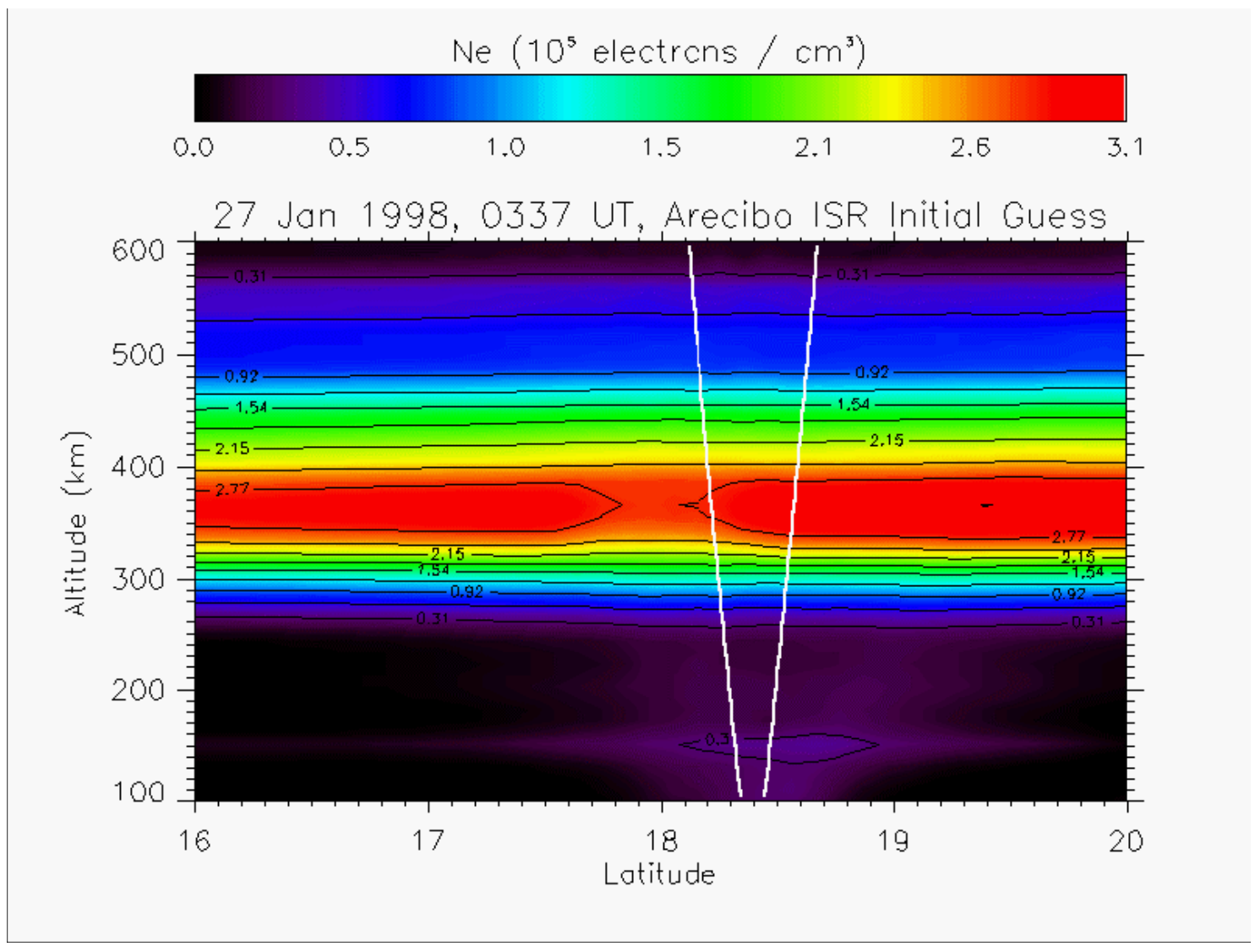


Figure 16c: Eastern Chain Reconstruction, ISR Initialization

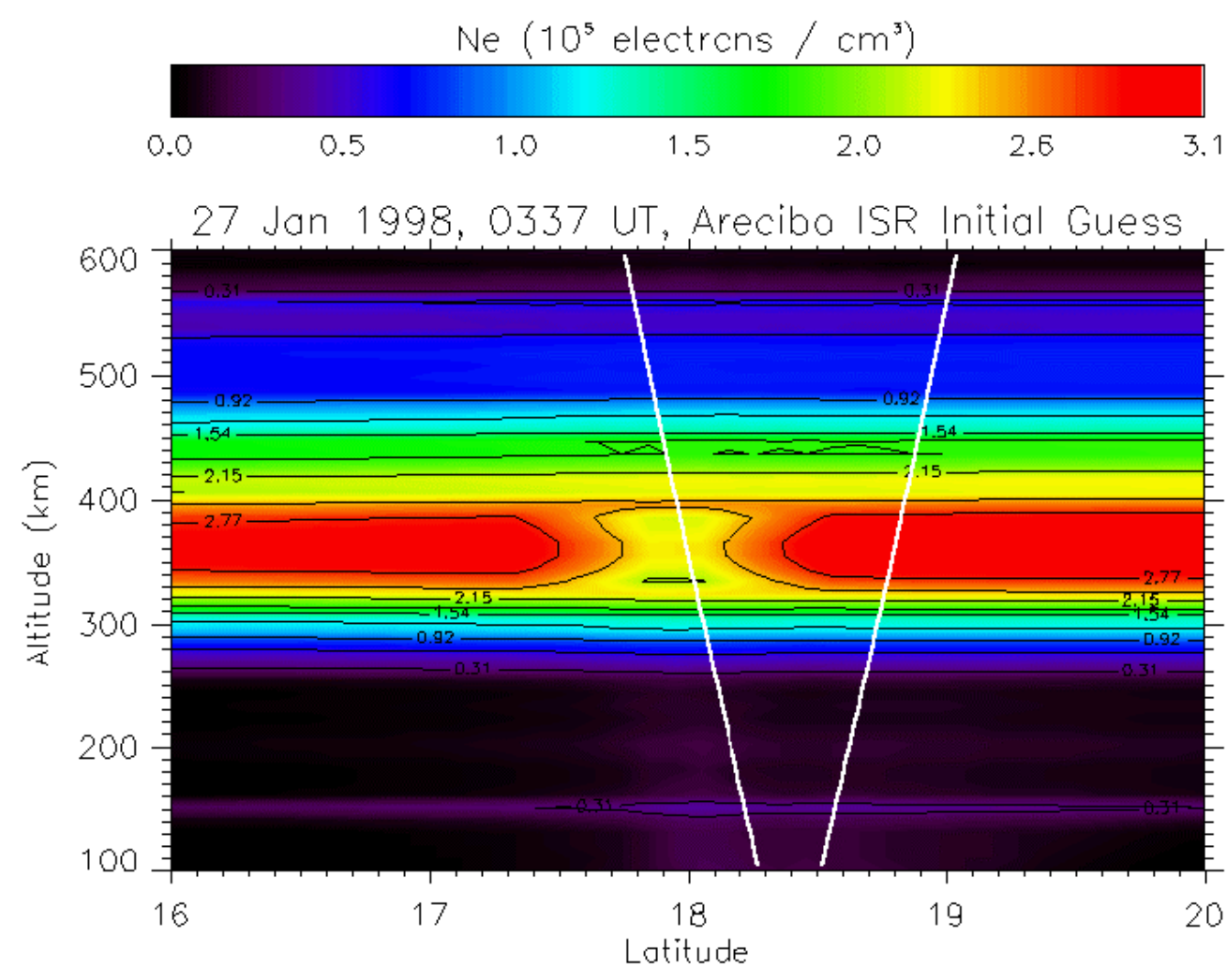


Figure 17: ISR Temperature Data and $\mathrm{n}_{\mathrm{e}}$ Scaling Factor

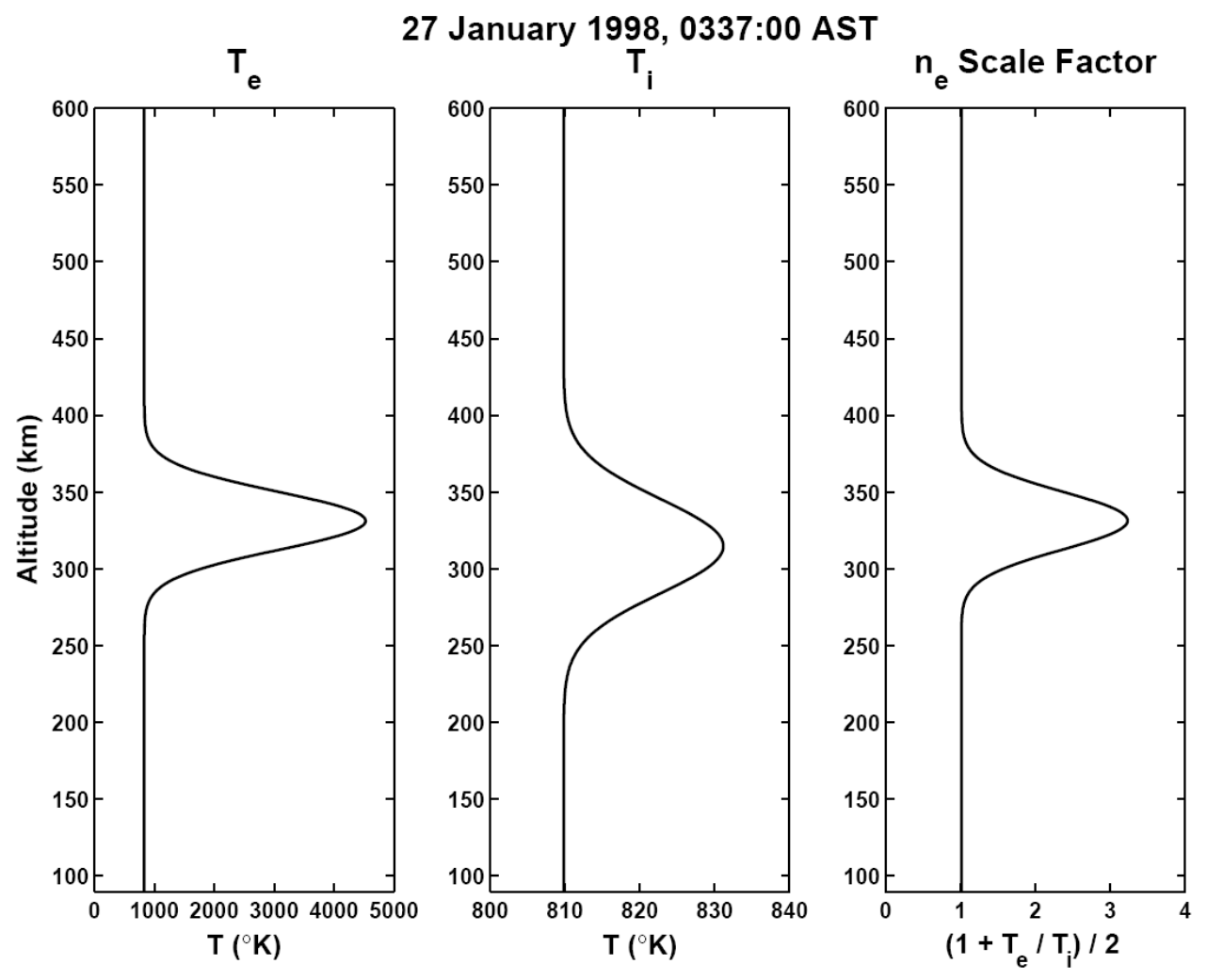


Figure 18: SAMI2 Background Electron Temperature

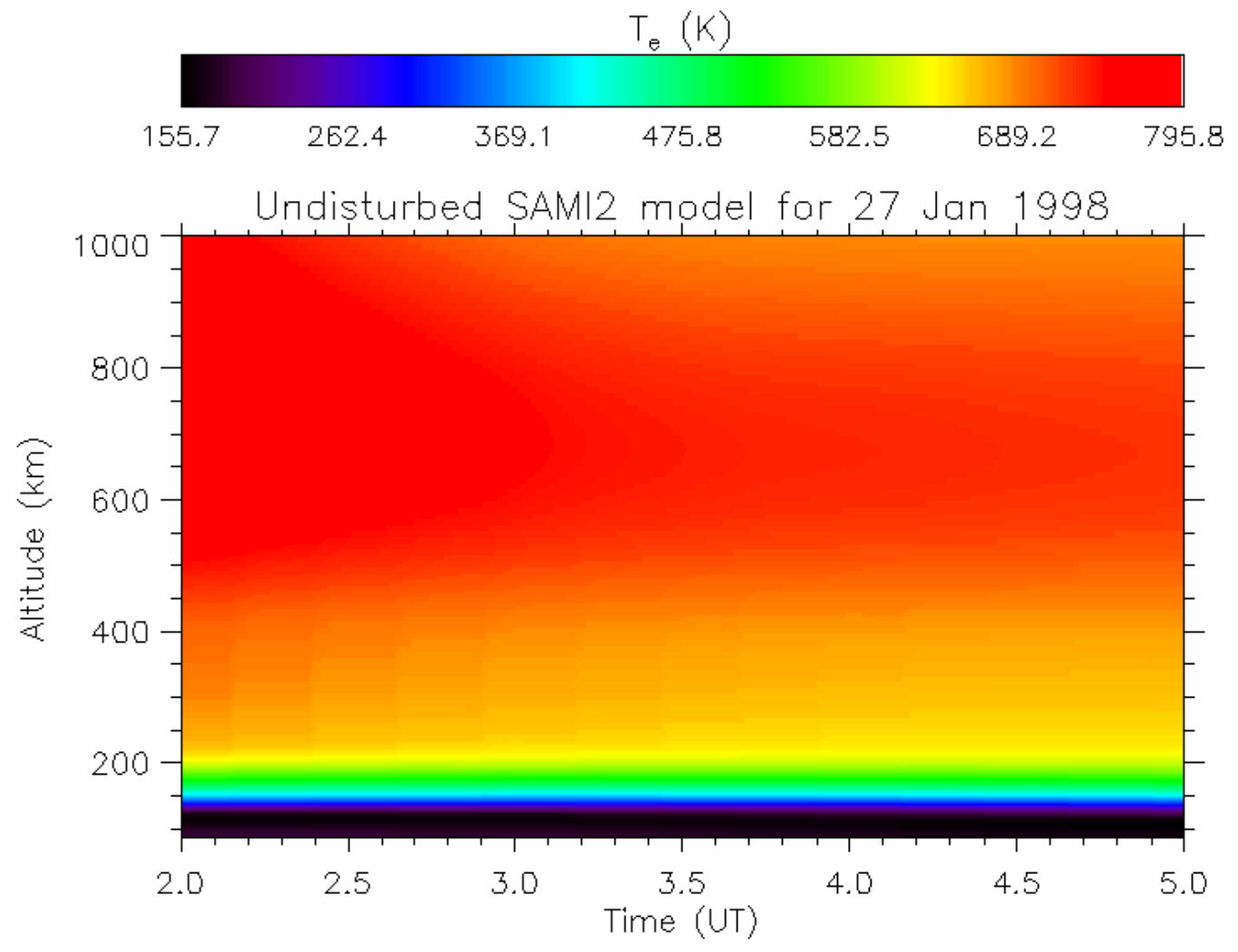


Figure 19: SAMI2 Heated Electron Temperature

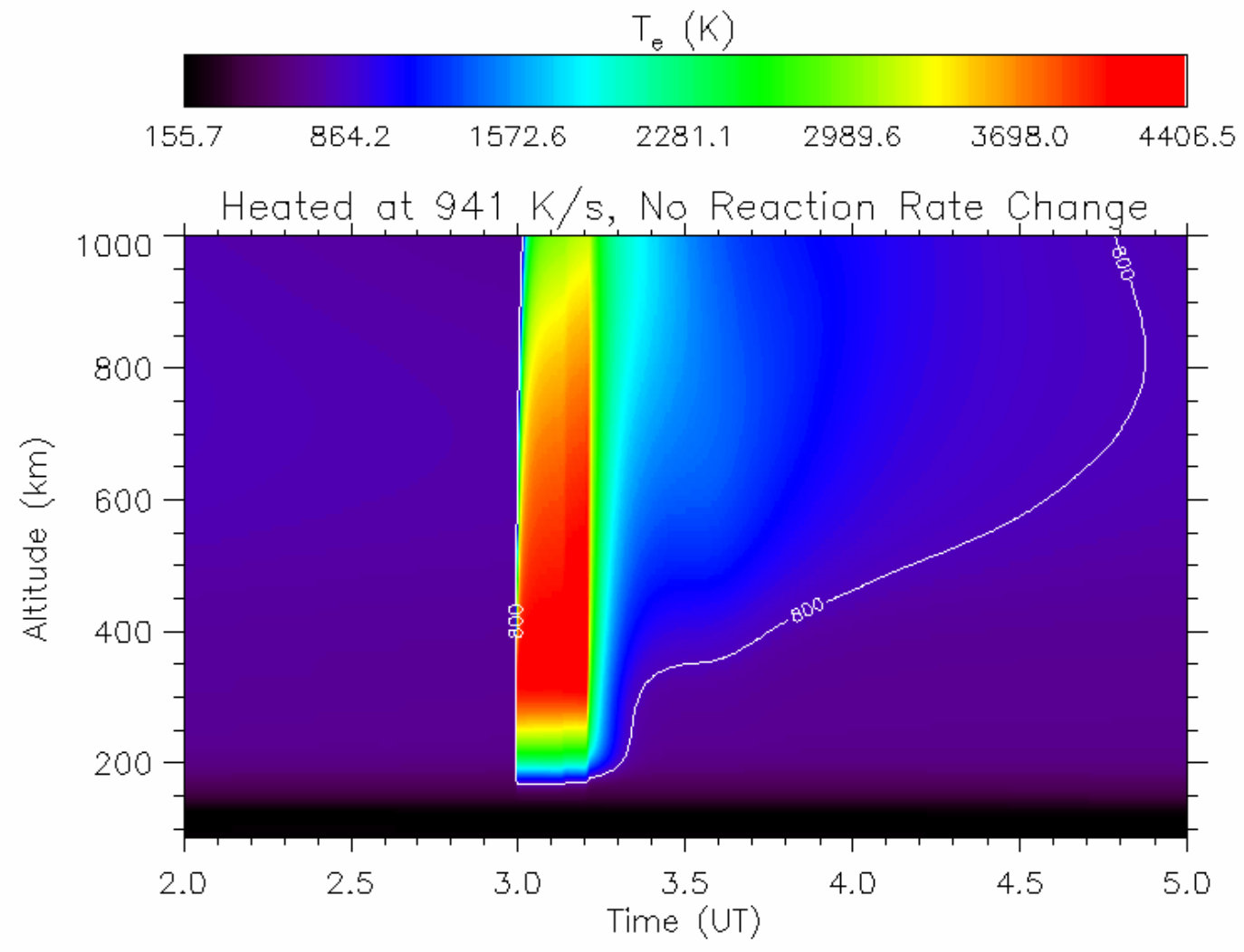


Figure 20: SAMI2 Change in Electron Temperature Due to Heating
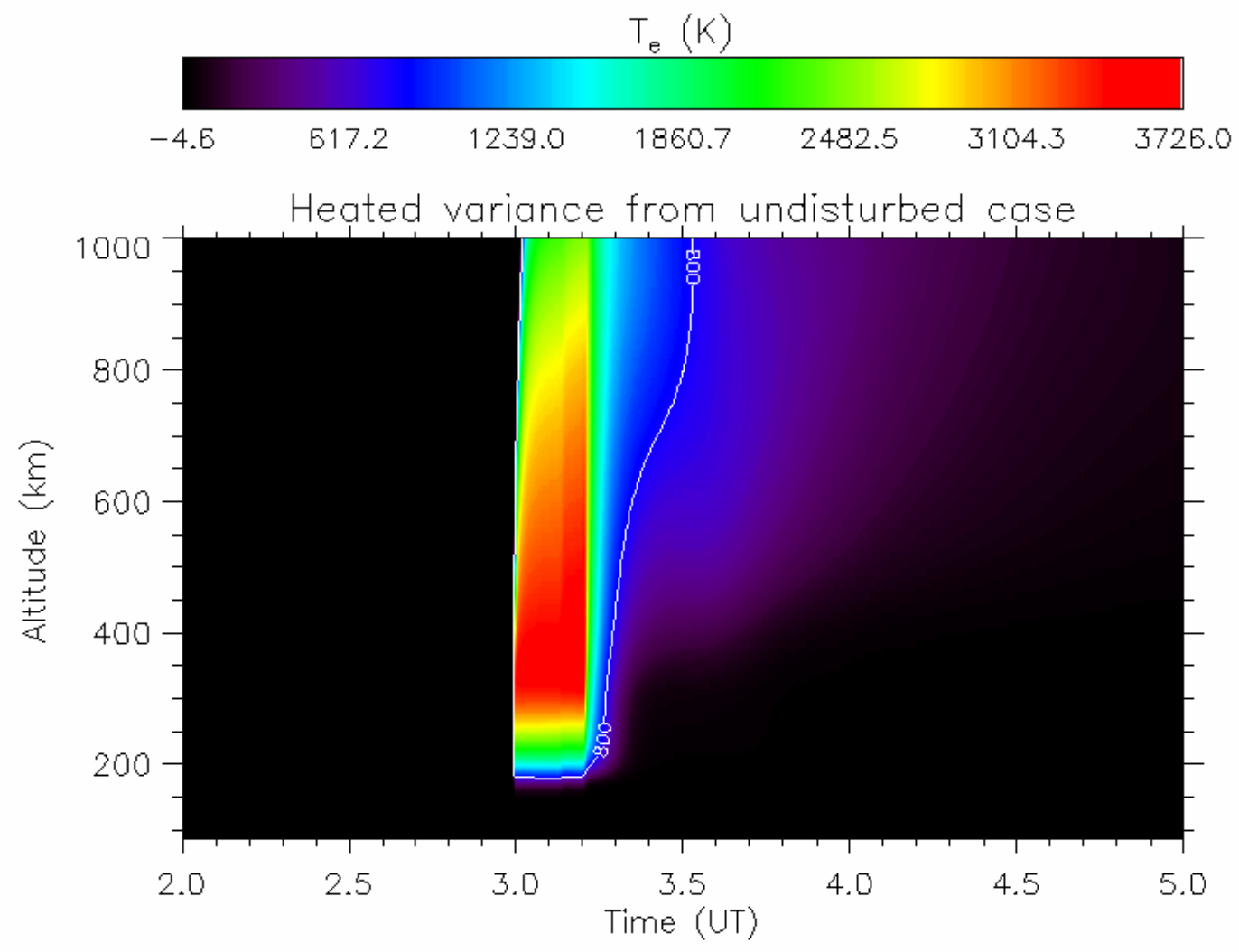
Figure 21: SAMI2 Background Electron Density

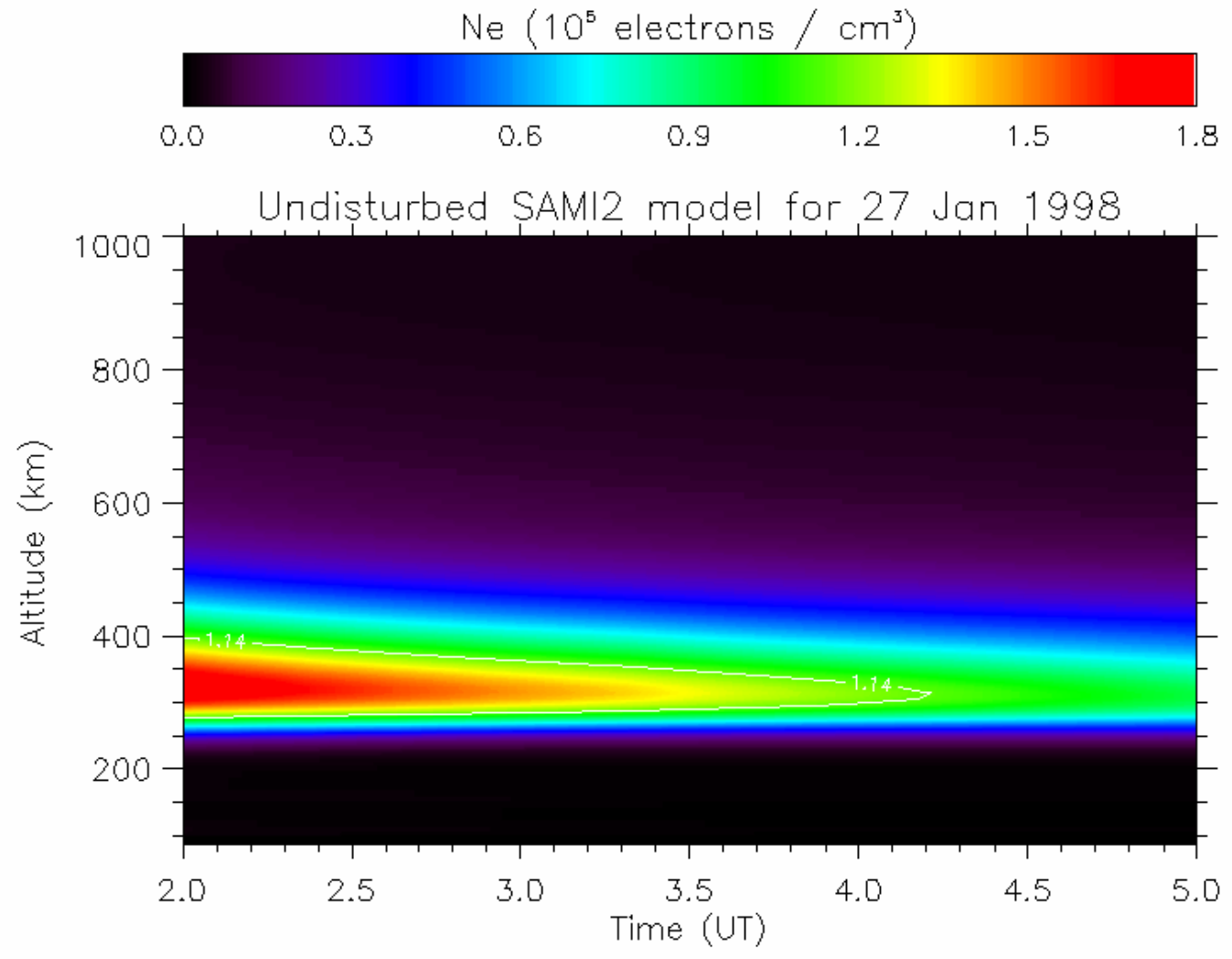


Figure 22: SAMI2 Heated Electron Density

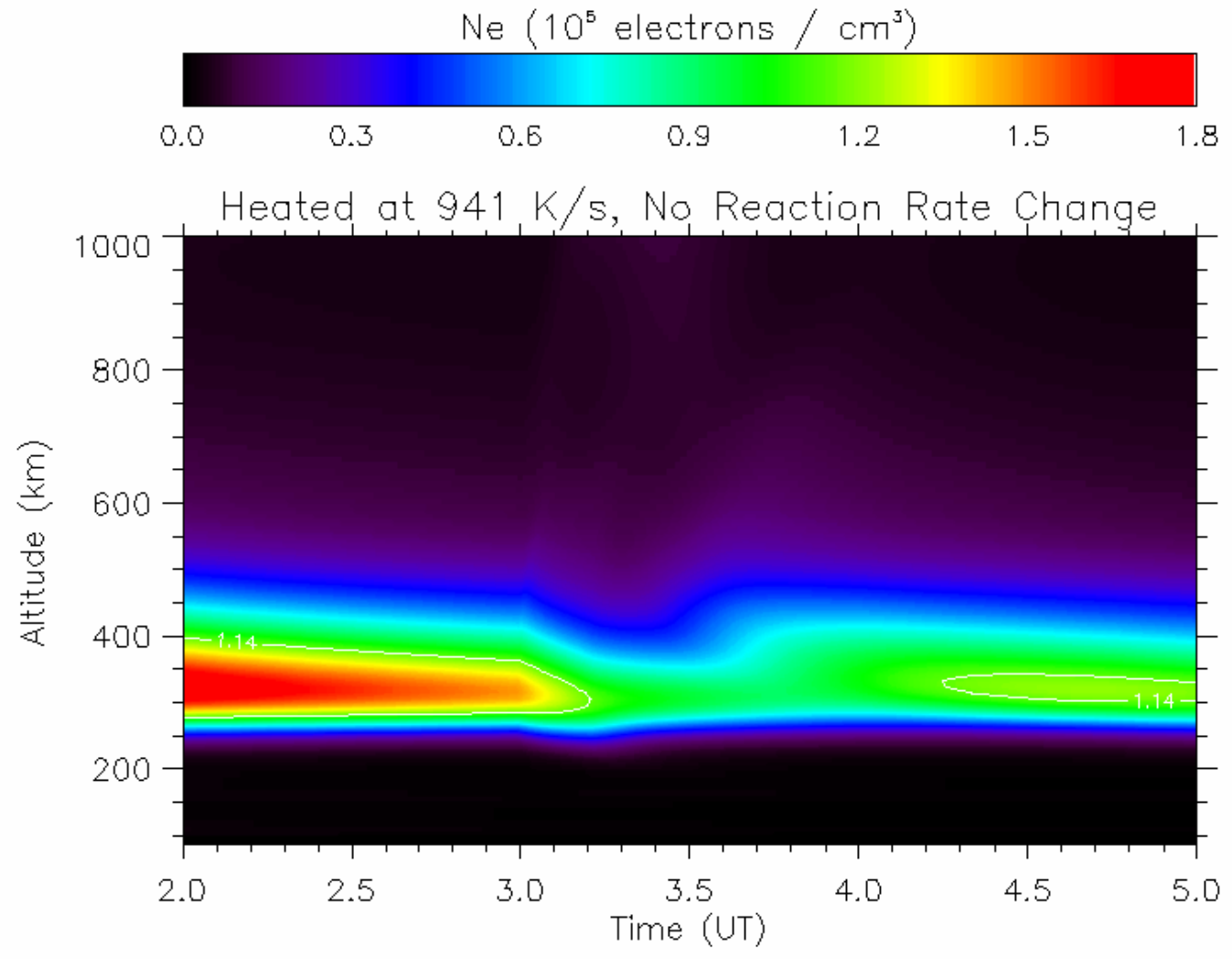


Figure 23: SAMI2 Change in Electron Density Due to Heating
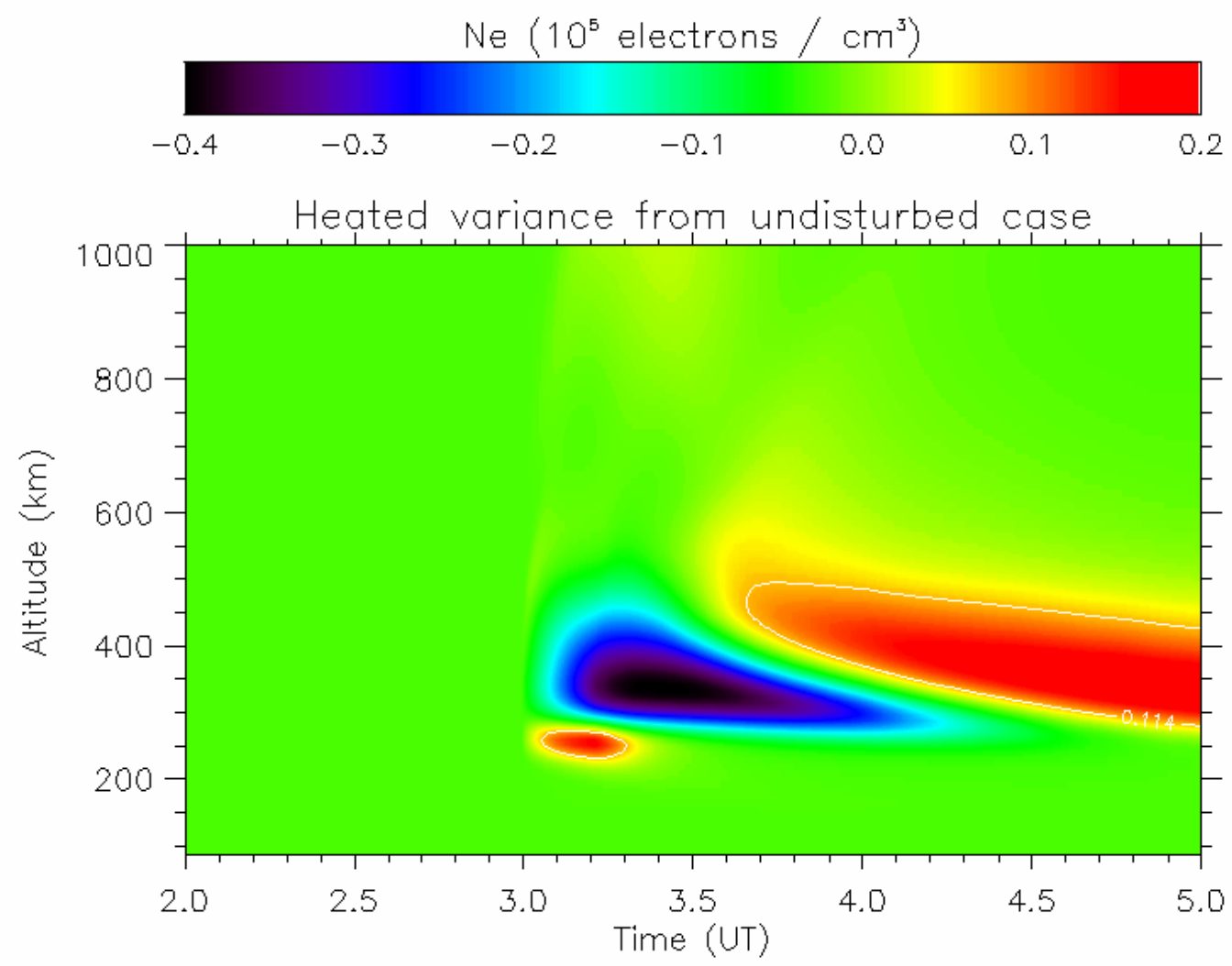
Figure 24: Change in Electron Density Due to Heating and Reaction Rate Effects

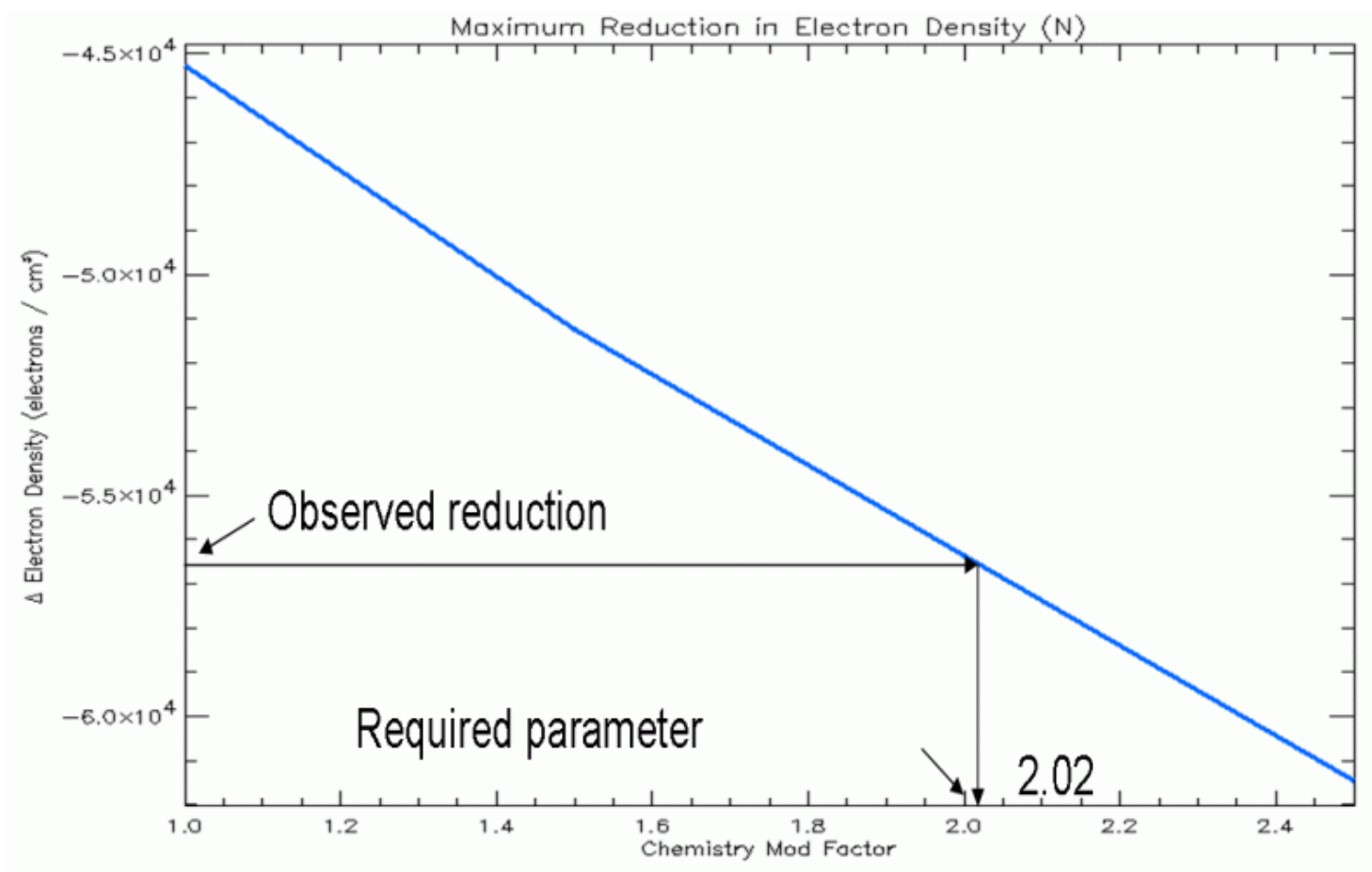


Figure 25: SAMI2 Final Modeling Result

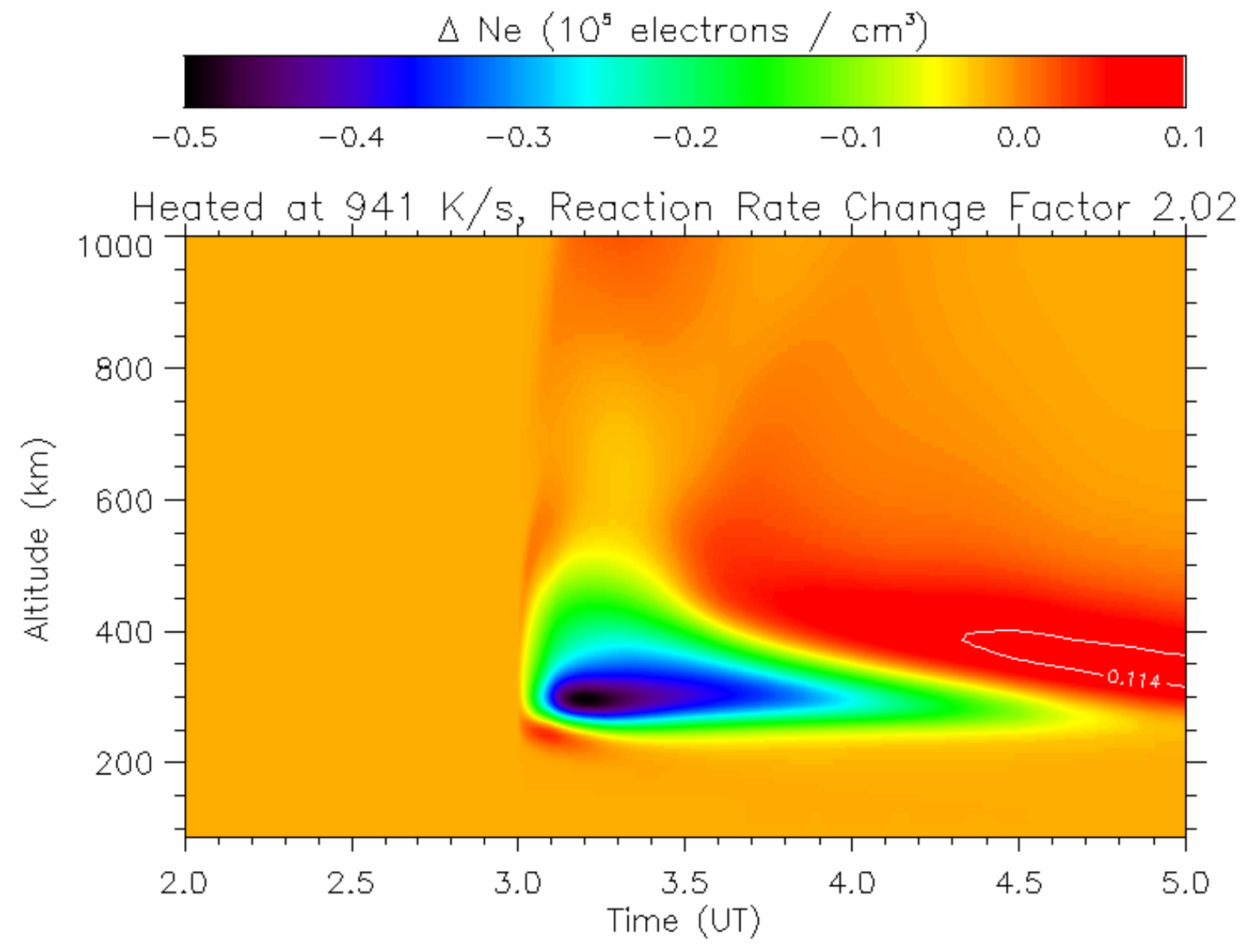


Figure 26: Chemistry Effect Comparison

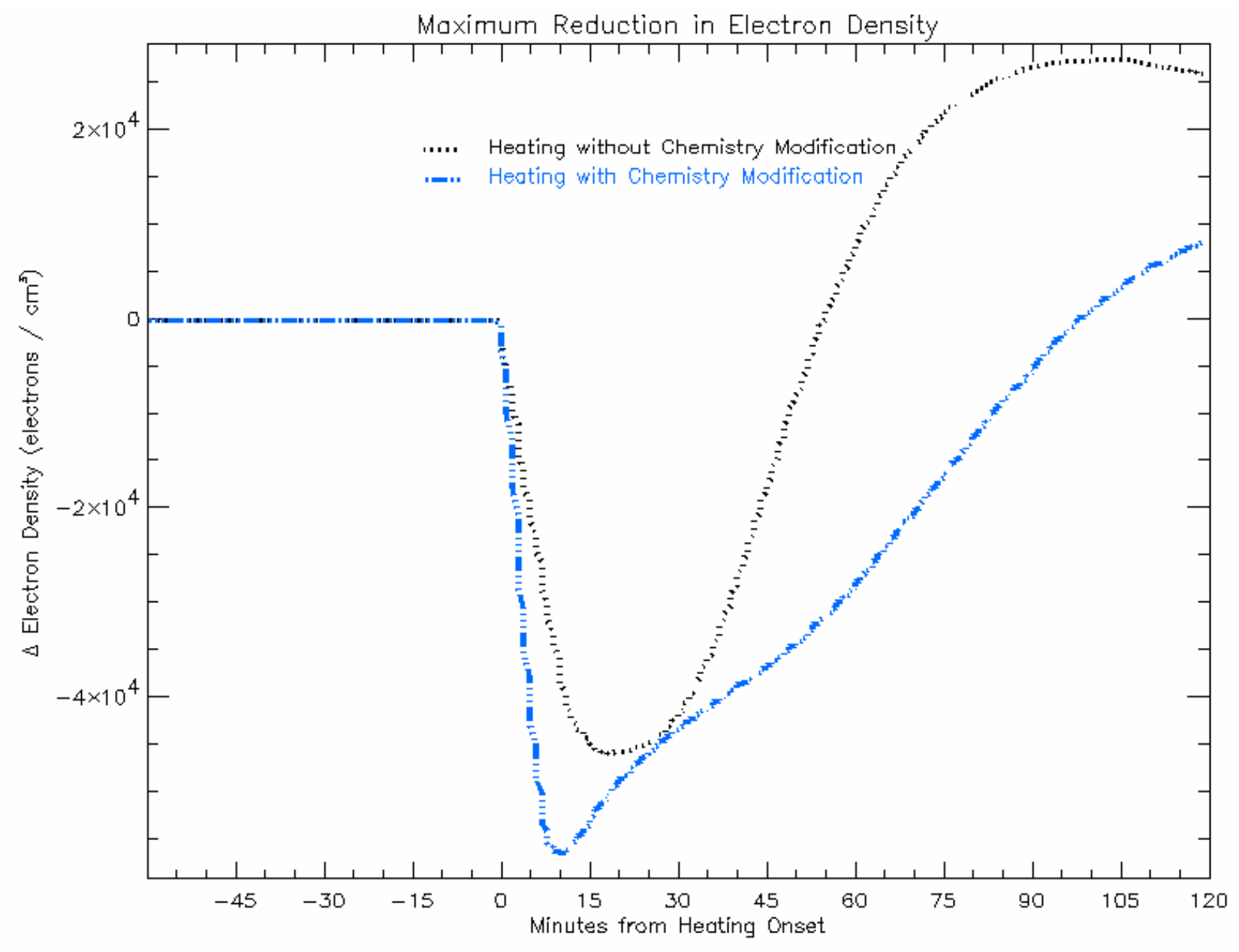




\section{APPENDIX A: CIT Reconstruction Software}

The Algebraic Reconstruction Technique (ART) based CIT code follows. It is written in the Interactive Data Language (IDL) developed by Research systems, Inc. IDL is based on the Fortran programming language, but has a number of features which make it an excellent tool for scientific research. In particular, operators and functions work on entire array without using loops, reducing programming time and making interactive data analysis more straightforward.

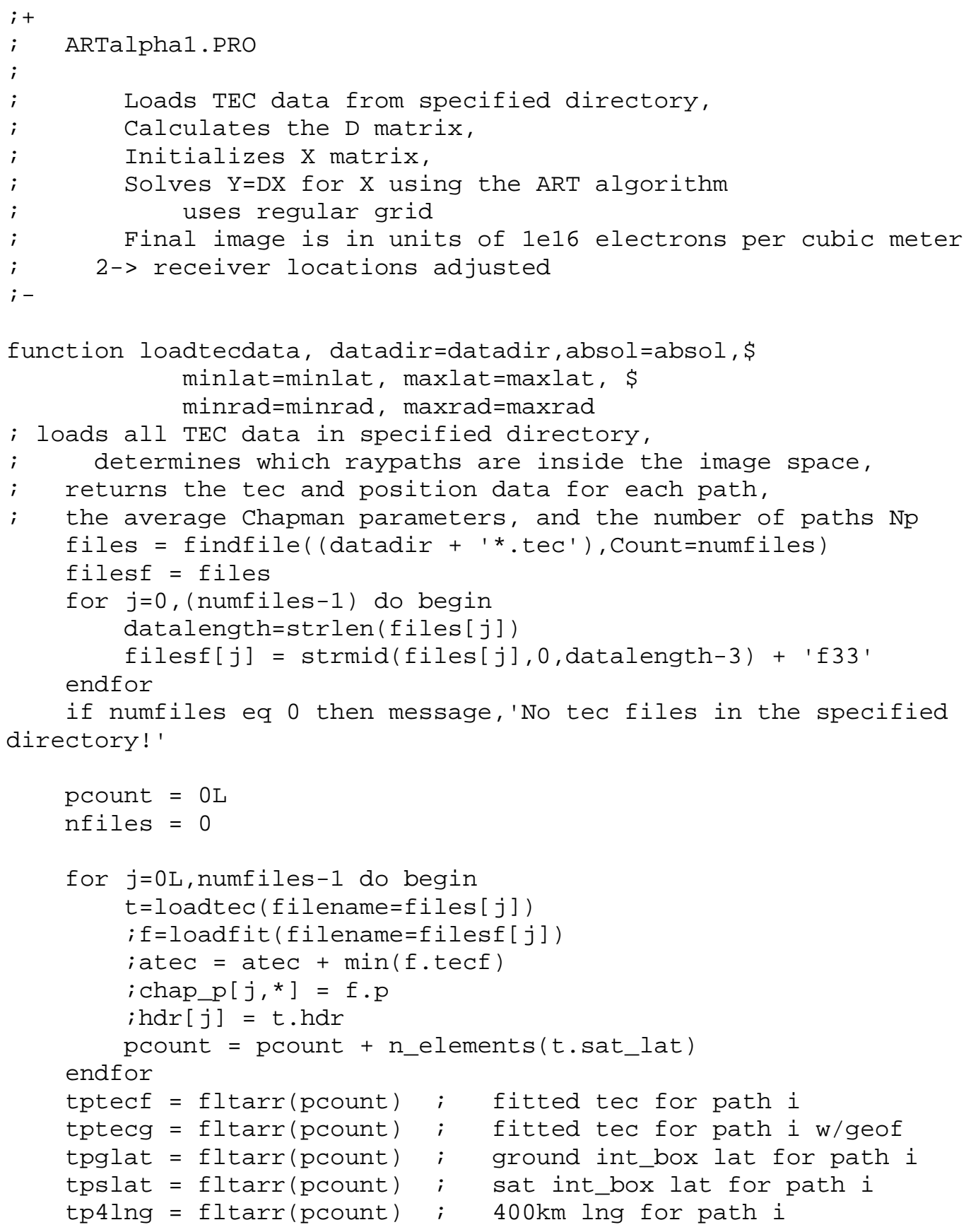




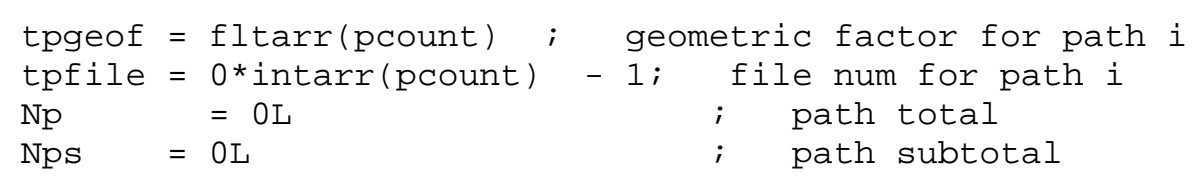




$$
\begin{aligned}
& )^{*} \text { tpgeof }[\mathrm{Np}] ; \text { TEC w/geof } \\
& \operatorname{tptecg}[N p]=1 e 12 *(t \cdot \operatorname{tecs}[i]+\min (f \cdot \operatorname{tec} f) \\
& \text { endelse } \\
& \text { tp4lng }[\mathrm{Np}]=\mathrm{t} . \operatorname{lng} \_400[\mathrm{i}] \\
& N p=N p+1 \\
& \text { tpfile }[\mathrm{Np}]=\mathrm{j}
\end{aligned}
$$

endif

endfor

if (Nps ne NP) then begin

nfiles $=$ nfiles +1

lbound $1[j]=$ Nps ; lower path bound for file $j$ endif ubound $1[j]=N p-1 ;$ upper path bound for file $j$

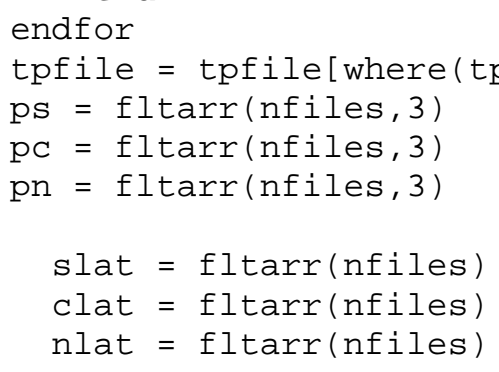




$\begin{array}{cc}\text { pc } & \text { : pc, } \$ \\ \text { pn } & \text { : pn, } \$ \\ \text { slat } & \text { : slat, } \$ \\ \text { clat } & \text { : clat, } \$ \\ \text { nlat } & \text { : nlat, } \$ \\ \text { ptecf } & \text { : ptecf, } \$ \\ \text { ptecg } & \text { : ptecg, } \$ \\ \text { pglat } & \text { : pglat, } \$ \\ \text { pslat } & \text { : pslat, } \$ \\ \text { pgeof } & : \text { pgeof, } \$ \\ \text { p4lng } & : \text { p4lng, } \$ \\ \text { nfiles } & : \text { nfiles, } \$ \\ \text { Np } & : \text { Np, } \$ \\ \text { pfile } & : \text { pfile } \$ \\ \text { END } & \end{array}$

function calcpaths, lgrid, nbl, rgrid, nbr, pglat, pslat, Np, Nb, \$ plotpaths=plotpaths, ith=ith, cith=cith

1 if n_elements(plotpaths) eq $\odot$ then plotpaths $=\odot$; else plotpaths $=$

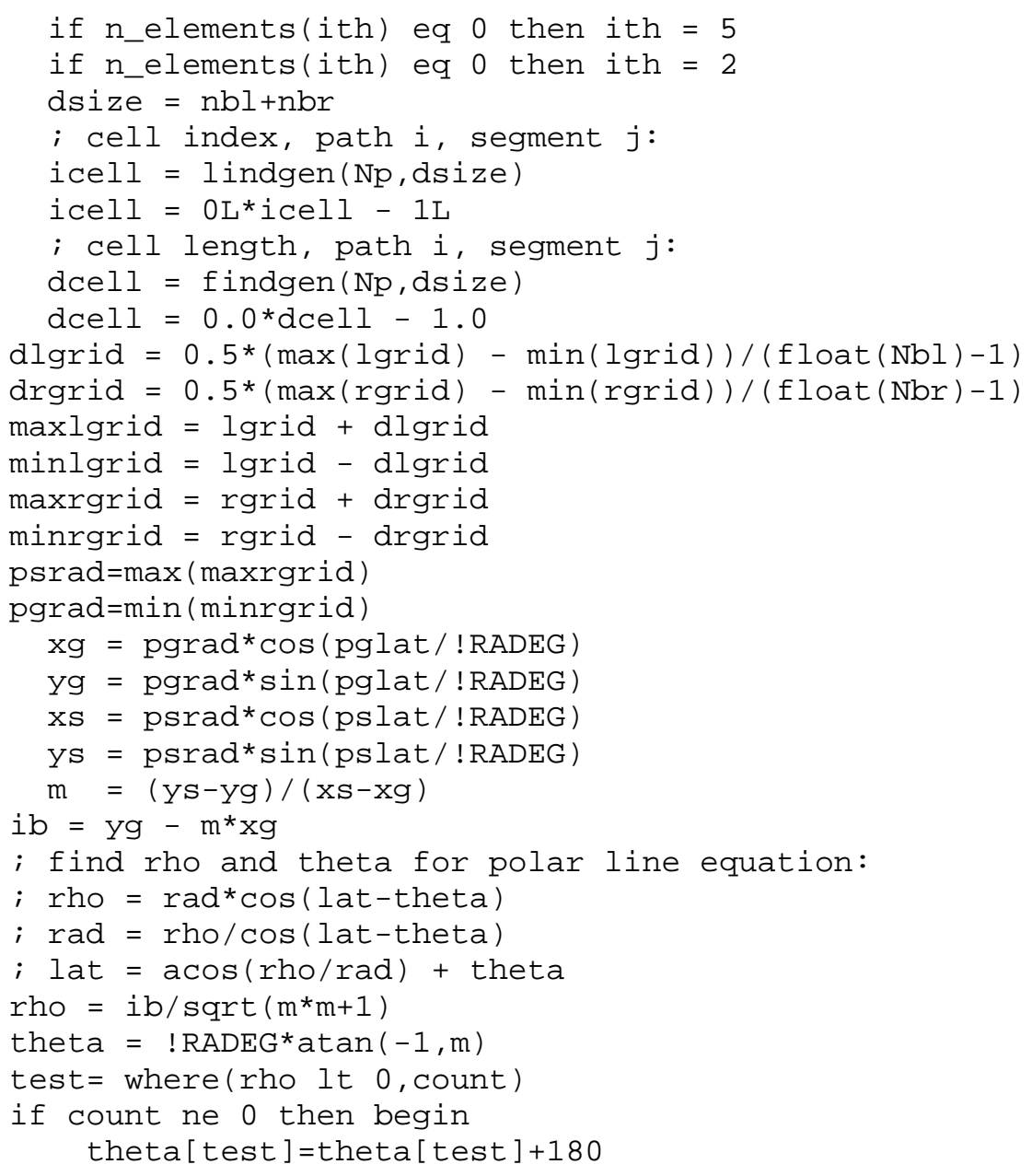


endif

rho $[$ test $]=\operatorname{abs}($ rho $[$ test $])$

test $=$ where (theta lt $\odot$, count $)$

if count ne $\odot$ then begin

endif

theta[test] $=$ theta[ test ] +360

rint=indgen $(\mathrm{Nbr})$

lint=indgen $(\mathrm{Nbl})$

$r v=$ dindgen $(\mathrm{Nbr}+1)$

$l v=$ dindgen $(\mathrm{Nbl}+1)$

$\mathrm{rv}[0:(\mathrm{Nbr}-1)]=\operatorname{double}\left(\min r g r i d\left[\mathrm{Nbl}^{*} \mathrm{rint}\right]\right) ;$ grid radius values

$\operatorname{rv}[\mathrm{Nbr}]=\operatorname{double}(\max (\max r g r i d))$

$\operatorname{lv}[\Theta:(\mathrm{Nbl}-1)]=$ double(minlgrid[lint $]) \quad$;grid latitude values

$\operatorname{lv}[\mathrm{Nbl}]=\operatorname{double}(\max (\max l g r i d))$

if plotpaths then begin

set_plot, 'PS'

device, filename=' c: \research \working \pathplot.ps', /inches, $x$ size=

10 , ysize $=7.5, /$ landscape, $x$ offset $=.5$, yoffset $=10.5$, /color

loadct, 0

; window, 2, xsize $=900$, ysize $=700$

plot, pglat, pglat,/nodata, \$

6378], yticks=1, \$

/ystyle, yrange $=[\min (\operatorname{minrgrid})-6378, \max (\operatorname{maxrgrid})-$

endif

/xstyle, $x$ range $=[\min (\operatorname{minlgrid}), \max (\max l g r i d)], x$ ticks $=1, \$$

title $=$ 'Paths through the image plane', \$

xtitle $=$ 'Latitude', $\$$

ytitle $=$ 'Altitude $(\mathrm{km}){ }^{\prime}$

for $i=0,(\mathrm{~Np}-1)$ do begin

; latitude where path $i$ intercepts each gridded radius:

if (pslat[i]-pglat[i] gt $\odot$ ) then begin

lat_rgrid $=\operatorname{double}($ theta $[i]+$ !RADEG*acos $(r h o[i] / r v))$

endif else begin

!RADEG*acos (rho[i]/rv) )

lat_rgrid $=$ double $($ theta $[i]$ -

endelse

test $=$ where (lat_rgrid gt 90.0 , count $)$

if count gt $\odot$ then lat_rgrid[test] = lat_rgrid[test] -180.0

test $=$ where (lat_rgrid $1 \mathrm{t}-90.0$, count)

if count gt $\odot$ then lat_rgrid[test] = lat_rgrid[test] +180.0

; radii for those latitudes:

rad_rgrid = rv

; radii where path i intercepts each gridded latitude:

rad_lgrid $=\operatorname{double}(\operatorname{abs}(\operatorname{rho}[i] / \cos ((\operatorname{lv}-\operatorname{theta}[i]) / \operatorname{RADEG})))$

; latitudes for those radii:

if (pslat[i]-pglat[i] gt $\odot$ ) then begin lat_lgrid $=$ double $($ theta $[i]+$

!RADEG*acos (rho[i]/rad_lgrid))

endif else begin

lat_lgrid = double $($ theta $[i]$ -

!RADEG*acos (rho[i]/rad_lgrid))

endelse

test $=$ where $($ lat_lgrid gt 90.0, count $)$ 
if count gt $\odot$ then lat_lgrid[test] = lat_lgrid[test] -180.0

test $=$ where (lat_lgrid lt -90.0 , count)

if count gt $\odot$ then lat_lgrid[test] = lat_lgrid[test] +180.0

; now reduce them to be inside the image box

test $=$ where $($ (lat_rgrid ge $(\min (\operatorname{minlgrid})))$ and (lat_rgrid le

$(\max (\max l g r i d)))$ and $\$$

(rad_rgrid le $(\max (\max r g r i d)))$, count)

(rad_rgrid ge (min(minrgrid))) and

; if (count ne $\odot$ ) then begin

; endif

lat_rgrid=lat_rgrid[test]

rad_rgrid=rad_rgrid[test]

test $=$ where $($ lat_lgrid ge $(\min (\min l g r i d)))$ and

(lat_lgrid le $(\max (\max l g r i d)))$ and $\$$

(rad_lgrid le (max(maxrgrid))), count)

(rad_lgrid ge (min(minrgrid))) and

vert_flag $=0$

if (count ne $\Theta$ ) then begin

lat_lgrid=lat_lgrid[test]

rad_lgrid=rad_lgrid[test]

endif else begin

vert_flag $=1$

lat_lgrid $=100$

endelse

rad_lgrid $=100$

; collect cell intersections into sequential pairs:

if (vert_flag eq 1) then begin

lat_pgrid = double (lat_rgrid)

rad_pgrid = double (rad_rgrid)

endif else begin

lat_pgrid = double ([lat_rgrid, lat_lgrid] $)$

rad_pgrid = double ([rad_rgrid,rad_lgrid] $)$

endelse ; end vert_flag condition

sindex = sort $($ rad_pgrid $)$

lat_pgrid = lat_pgrid[sindex]

rad_pgrid $=$ rad_pgrid[sindex ]

; determine cells and $D$ values for each sequential pair $; d$-value $=\operatorname{sqrt}\left[\operatorname{ro}^{\wedge} 2+r 1^{\wedge} 2-2 r 1 r 2 \cos (\right.$ phi1-phi2 $\left.)\right]$

for $j=0$, (n_elements(lat_pgrid)-2) do begin

lat_seg $=0.5^{*}($ lat_pgrid[j] + lat_pgrid[j+1])

rad_seg $=0.5^{*}\left(\operatorname{rad} \_p g r i d[j]+\operatorname{rad} \_p g r i d[j+1]\right)$

ind $=$ where ( lat_seg ge minlgrid) AND $\$$

(lat_seg le maxlgrid) AND \$

(rad_seg ge minrgrid) AND \$

sind $=\operatorname{size}($ ind $)$

(rad_seg le maxrgrid))

if sind[0] ne 1 then print, 'ERROR: multi-

intersection: $\operatorname{dim}={ }^{\prime}$, sind $[0]$

icell $[i, j]=$ ind $[\Theta]$

$\operatorname{dcell}[i, j]=\operatorname{sqrt}\left(\operatorname{rad} \_\operatorname{pgrid}[j]{ }^{*} \operatorname{rad} \_\operatorname{pgrid}[j]+\right.$

rad_pgrid[j+1]*rad_pgrid[j+1] - 2*rad_pgrid[j] * rad_pgrid[j+1] ${ }^{*} \cos ($

(lat_pgrid[j] - lat_pgrid[j+1]) / !RADEG) )

endfor; icell loop

;print, i, vert_flag 
if plotpaths and ( $i$ mod ith eq $\odot$ ) then

oplot, lat_pgrid, rad_pgrid-6378

if $((i+1) \bmod 100$ eq $\odot)$ then print, 'Finished path ', $i+1$, '

of ', $\mathrm{Np}$

endfor ; path loop

if plotpaths then begin

; draw lat grid

for $k=0$, nbl-1, cith do $\$$

plots, [minlgrid $[\mathrm{k}], \operatorname{minlgrid}[\mathrm{k}]],[\min (\operatorname{minrgrid})-$

6378, $\max ($ maxrgrid) -6378$]$, color $=200$

plots, [max(maxlgrid), $\max (\max l g r i d)],[\min (\operatorname{minrgrid})$ -

6378, $\max ($ maxrgrid) -6378$]$, color $=200$

for $k=0, \mathrm{nbr}-1$, cith do $\$$

plots, [min(minlgrid), $\max (\max l g r i d)],\left[\operatorname{minrgrid}\left[k^{*} n b l\right]-\right.$

6378, minrgrid [k*nbl]-6378], color $=200$ plots, [min(minlgrid), $\max (\max l g r i d)],[\max (\max r g r i d)-$

6378, $\max ($ maxrgrid) -6378$]$, color $=200$ ; win2gif, filename=' c : \research \working \pathplot.gif' device,/close_file

endif set_plot, 'WIN'

print, 'done'

dcell $=$ dcell $* 1 \mathrm{e} 5$

$\mathrm{D}=\left\{\begin{array}{c}\text { icell } \\ \text { icell, } \$\end{array}\right.$

return, D

dcell : dcell\}

END

function chapinit, lats, rgrid,ps, pc, pn, slat, clat, nlat, nbl, nbr

chap $=\operatorname{reform}(\operatorname{rgrid}, \mathrm{nbl}, \mathrm{nbr})$

$\mathrm{cz}=\operatorname{reform}\left(\operatorname{chap}\left[\theta,{ }^{*}\right]\right)$

asize = n_elements (slat)

$\mathrm{pz}=$ findgen $\left(3^{*}\right.$ asize $)$

pne $=$ findgen $\left(3^{*}\right.$ asize $)$

$\mathrm{ph}=$ findgen $\left(3^{*}\right.$ asize $)$

plats $=$ findgen $\left(3^{*}\right.$ asize $)$

$p z[\odot: \operatorname{asize}-1]=p s\left[{ }^{*}, \Theta\right]$

pz $\left[\right.$ asize: $2^{*}$ asize -1$]=\operatorname{pc}\left[{ }^{*}, 0\right]$

$\mathrm{pz}\left[2^{*}\right.$ asize $\left.: 3^{*} \operatorname{asize}-1\right]=\mathrm{pn}\left[{ }^{*}, 0\right]$

pne $[0: \operatorname{asize}-1]=\operatorname{ps}\left[{ }^{*}, 1\right]$

pne [asize:2*asize-1] $=$ pc $\left[{ }^{*}, 1\right]$

pne $\left[2{ }^{*}\right.$ asize: $3^{*}$ asize -1$]=\operatorname{pn}\left[{ }^{*}, 1\right]$

$\operatorname{ph}[0: \operatorname{asize}-1]=\operatorname{ps}[*, 2]$

$\operatorname{ph}\left[\right.$ asize: $2^{*}$ asize -1$]=\operatorname{pc}\left[{ }^{*}, 2\right]$

$\operatorname{ph}\left[2^{*}\right.$ asize $: 3^{*}$ asize -1$]=\operatorname{pn}\left[{ }^{*}, 2\right]$

plats $[0: \operatorname{asize}-1]=\operatorname{slat}[*, 0]$

plats [asize: $2 *$ asize-1] $=\operatorname{clat}[*, 0]$

plats $\left[2{ }^{*}\right.$ asize: $3^{*}$ asize -1$]=\operatorname{nlat}\left[{ }^{*}, 0\right]$ 


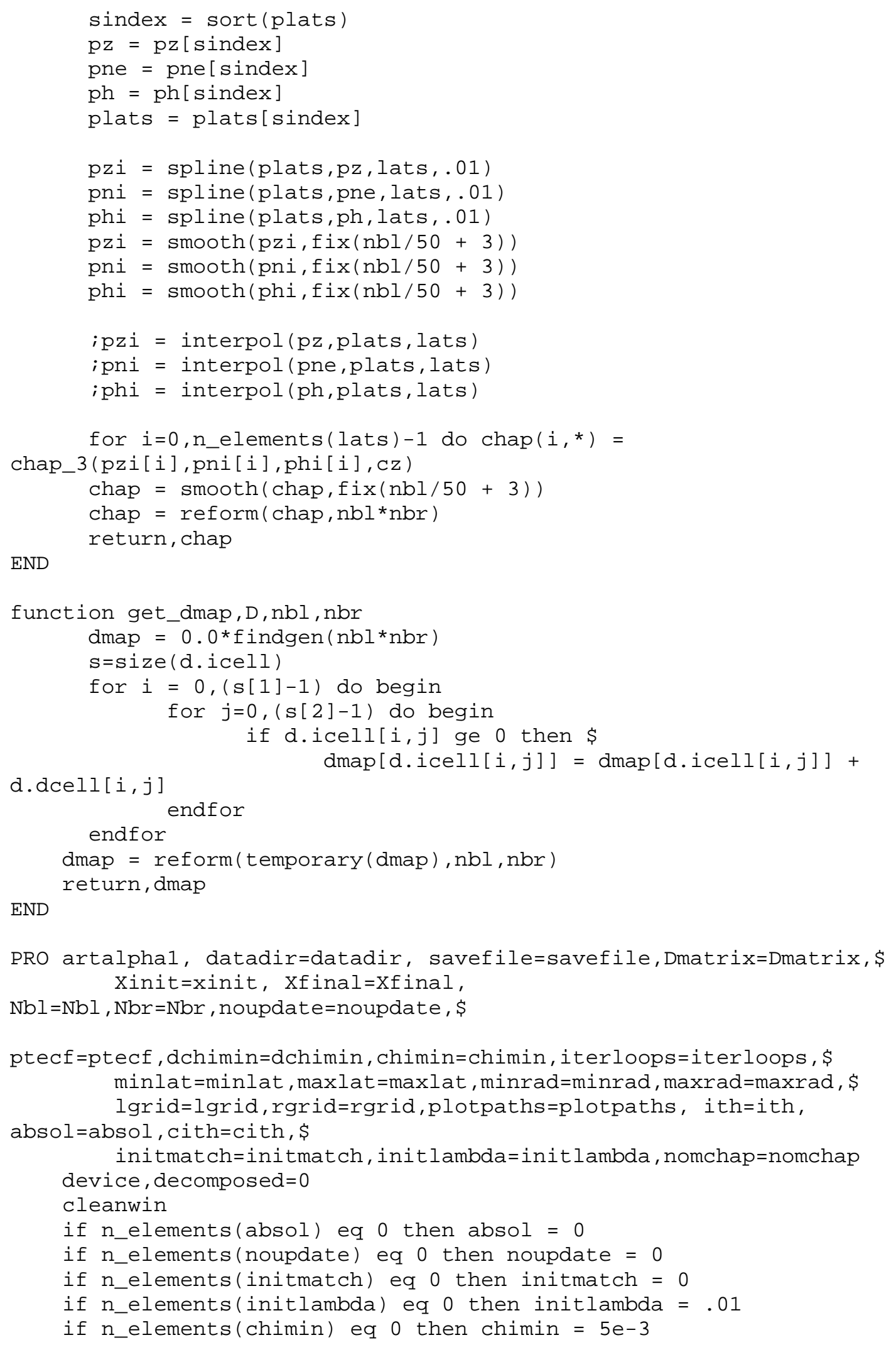

PRO artalpha1, datadir=datadir, savefile=savefile, Dmatrix=Dmatrix, $\$$ Xinit=xinit, Xfinal=xfinal, 


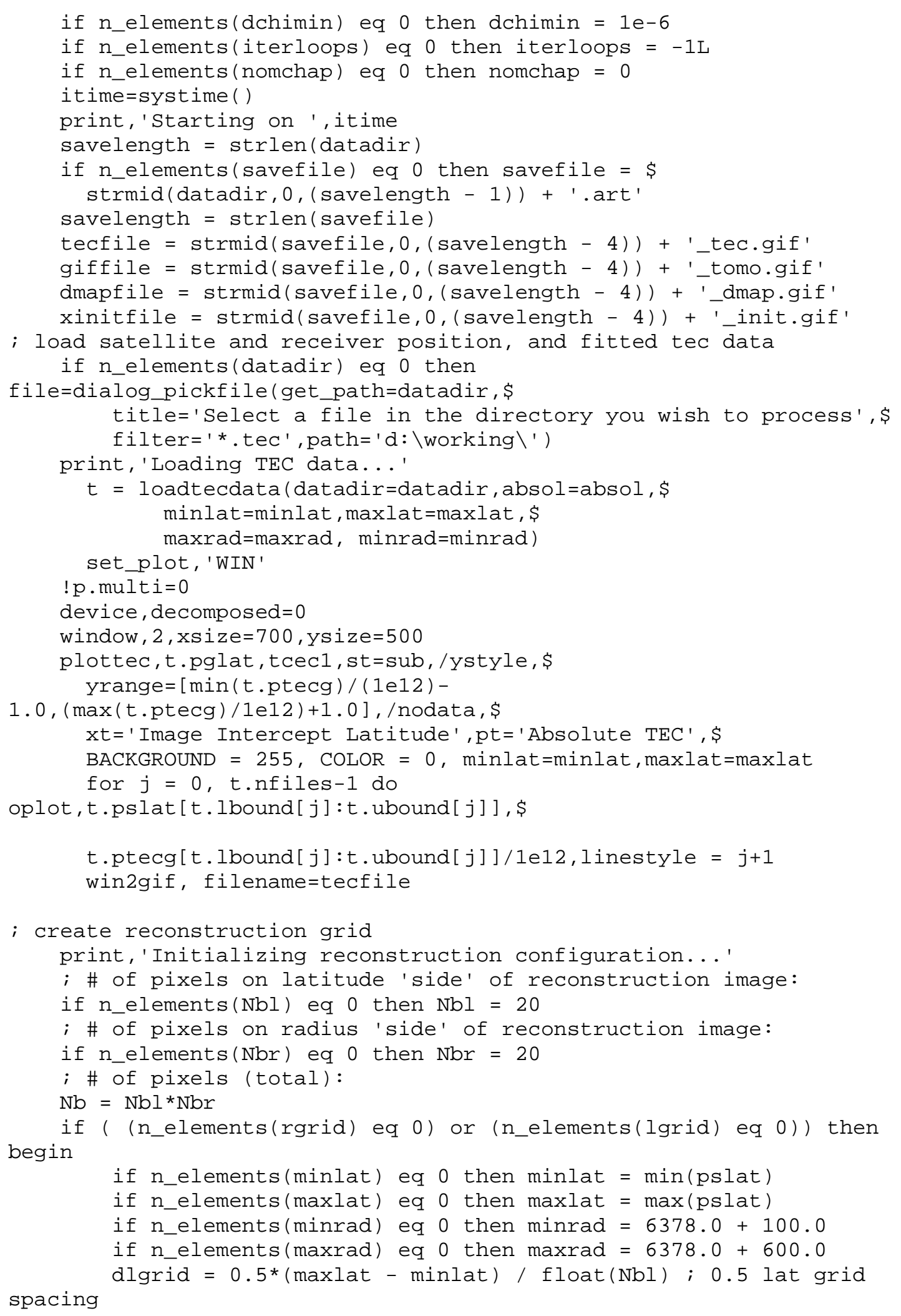


spacing

drgrid $=0.5^{*}(\operatorname{maxrad}-\operatorname{minrad}) /$ float $(\mathrm{Nbr}) ; 0.5 \mathrm{rad}$ grid

; lat coords of center of the box around each pixel:

lgrid $=$ fltarr $(\mathrm{Nbl} * \mathrm{Nbr})$

; rad coords of center of the box around each pixel:

rgrid = lgrid

bi $=$ indgen $\left(\mathrm{Nbl}^{*} \mathrm{nbr}\right)$

bl $=(b i \bmod \mathrm{Nbl})$

lgrid $\left[{ }^{*}\right]=2{ }^{*} b l^{*} d l g r i d+d l g r i d+$ minlat

br $=$ floor $(b i / N b l)$

$\operatorname{rgrid}\left[{ }^{*}\right]=2{ }^{*} b{ }^{*}$ drgrid + drgrid $+\operatorname{minrad}$

undefine, bi

undefine, bl

undefine, $b r$

endif else begin

minlat $=\min ($ lgrid)

maxlat $=\max ($ lgrid $)$

minrad=min ( $r g r i d)$

endelse

$\operatorname{maxrad}=\max ($ rgrid $)$

dtstamp $=$ ss $2 \mathrm{hhmm}(\mathrm{t} \cdot \mathrm{hdr}[0,8])+{ }^{\prime}$ on DOY ' $+\$$

strcompress(string(fix (t.hdr $[0,7])), /$ remove_all) $+\$$

' of ' + strcompress(string(fix $(\operatorname{t.hdr}[\odot, 6]))$,/remove_all)

; initialize reconstruction configuration

stime=systime ()

print, 'Calculating Dmatrix of ',t.Np,' paths through pixel grid

on: ', stime

if n_elements(Dmatrix) ne $\odot$ then $s=s i z e(D m a t r i x . d c e l l)$ else

$\mathrm{s}=[\odot, \Theta, \Theta]$

dsize $=n b l+n b r$

if $((s[1]$ ne t.Np) or (s[2] ne (dsize))) then begin

print, '...performing calculations'

$\mathrm{D}=$

calcpaths(lgrid, Nbl, rgrid, Nbr, t.pglat, t.pslat, t. Np, Nb, plotpaths=plotpa

ths, ith=ith, cith=cith)

endif else begin

print, '...Using loaded Dmatrix array'

endelse

D = Dmatrix

stime=systime ()

print, 'Finished Dmatrix calculation on

: ', stime

dmap = get_dmap $(D, n b l, n b r)$

loadct, 39

window, $\odot$, xsize $=795$, ysize $=543$, title $=$ ' Dmap ',$x p o s=0$, ypos $=\odot$

erase, 255

plotrecon, dmap, rgrid, nbr, lgrid, nbl, ptitle='Path Density

Map', btitle='Total path length per cell $(\mathrm{km})$ '

; xyouts, .3, .82, dtstamp,/normal, color $=0$, charsize $=1.5$

win2gif, filename $=$ dmapfile

window, $\odot, x$ size $=395$, ysize $=540$, title $=$ ' Dmap ',$x p o s=0$, ypos $=0$

erase, 0

plotrecon, dmap, rgrid, nbr, lgrid, nbl, ptitle='Path Density

Map', btitle='Total path length per cell $(\mathrm{km})$ '

; xyouts, .3, .82, dtstamp, /normal, color $=255$, charsize $=1.5$ 


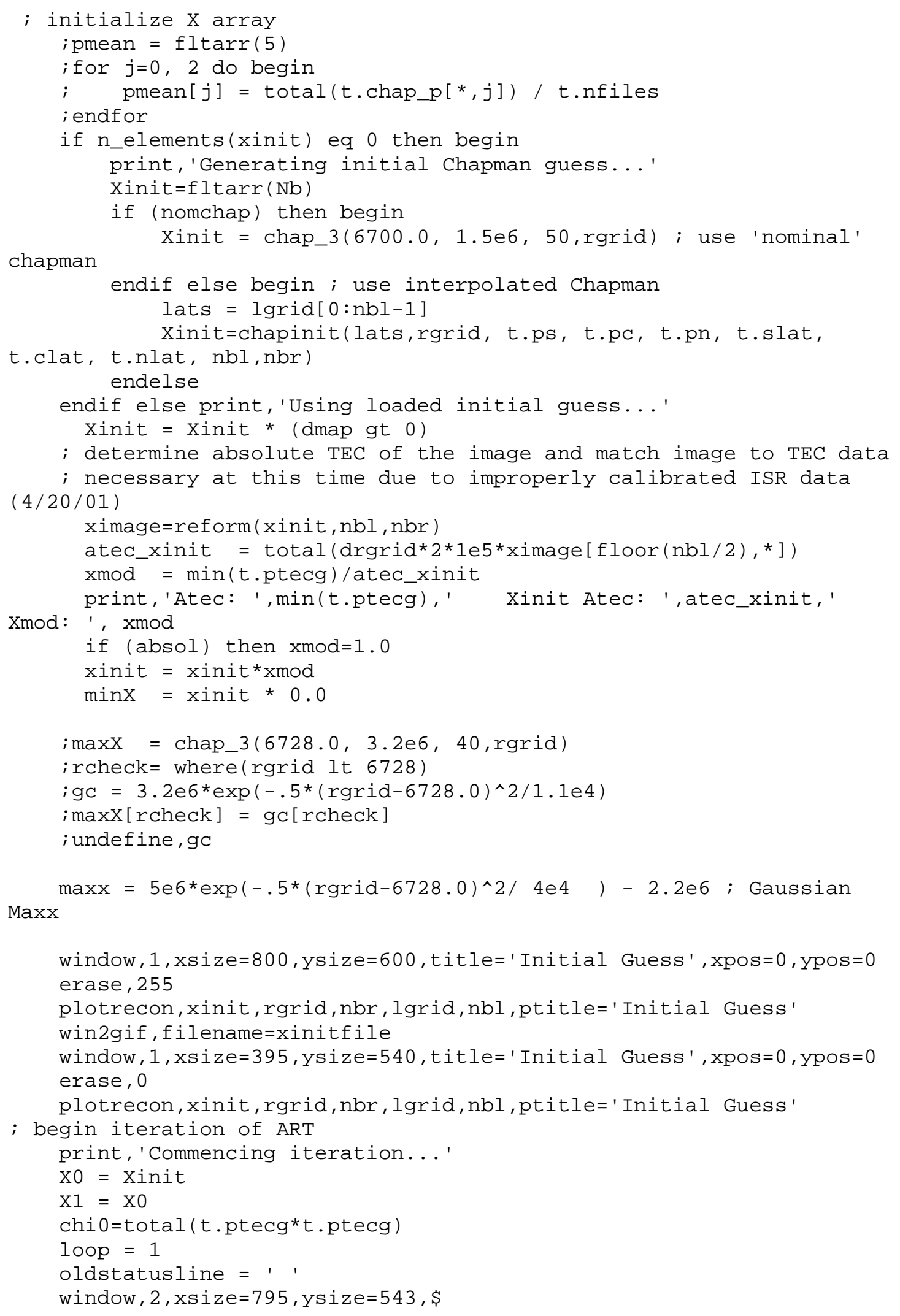




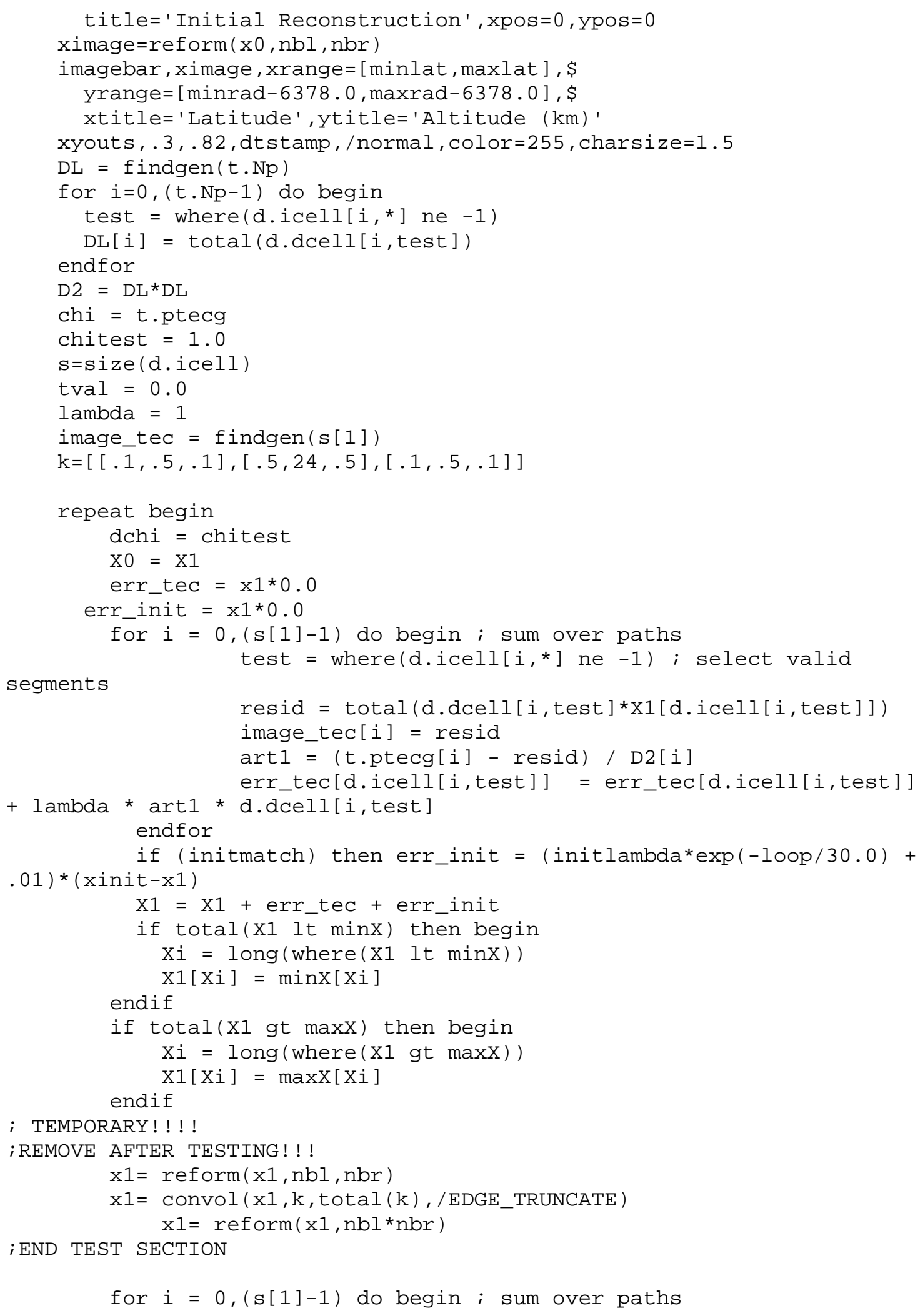




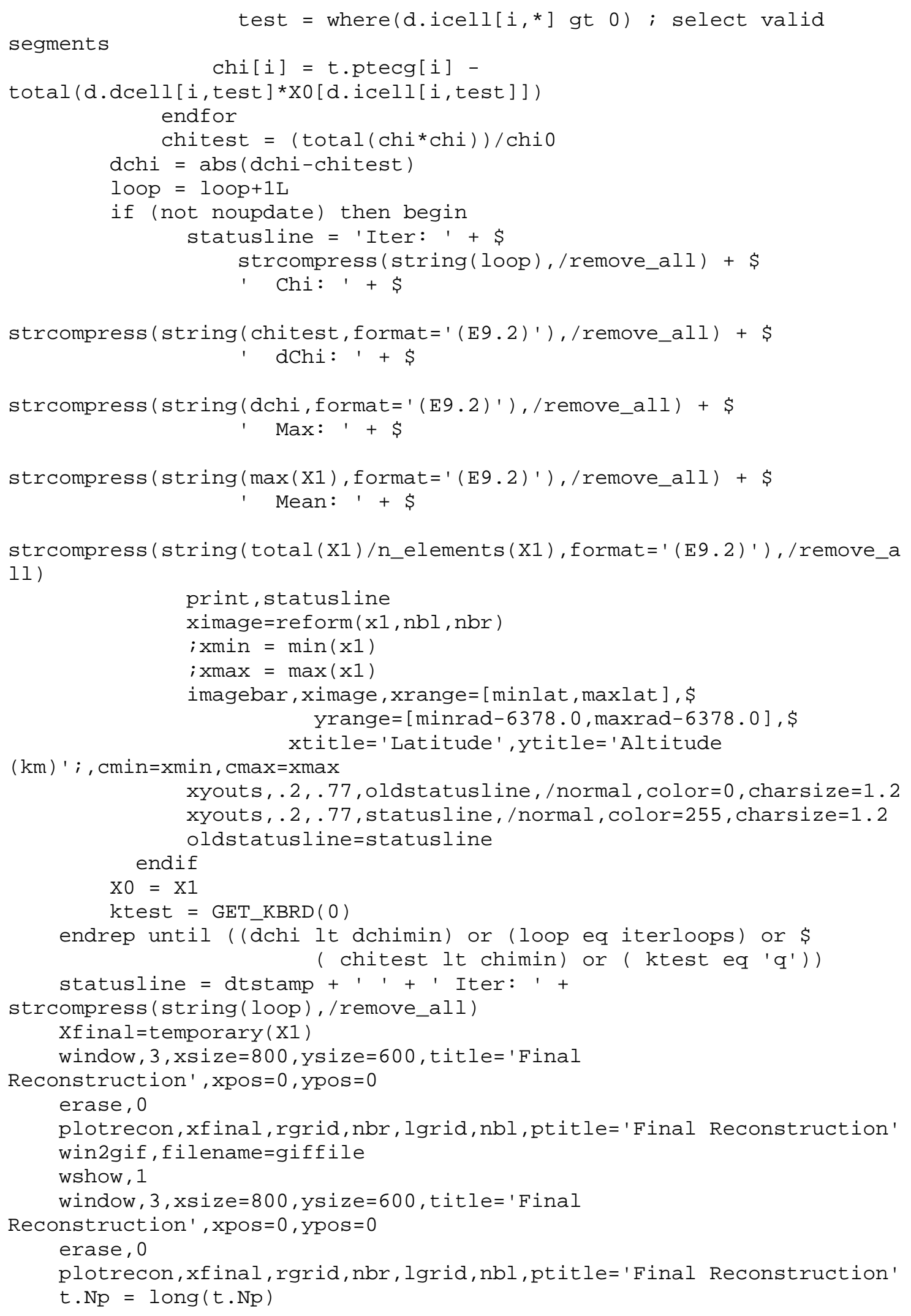




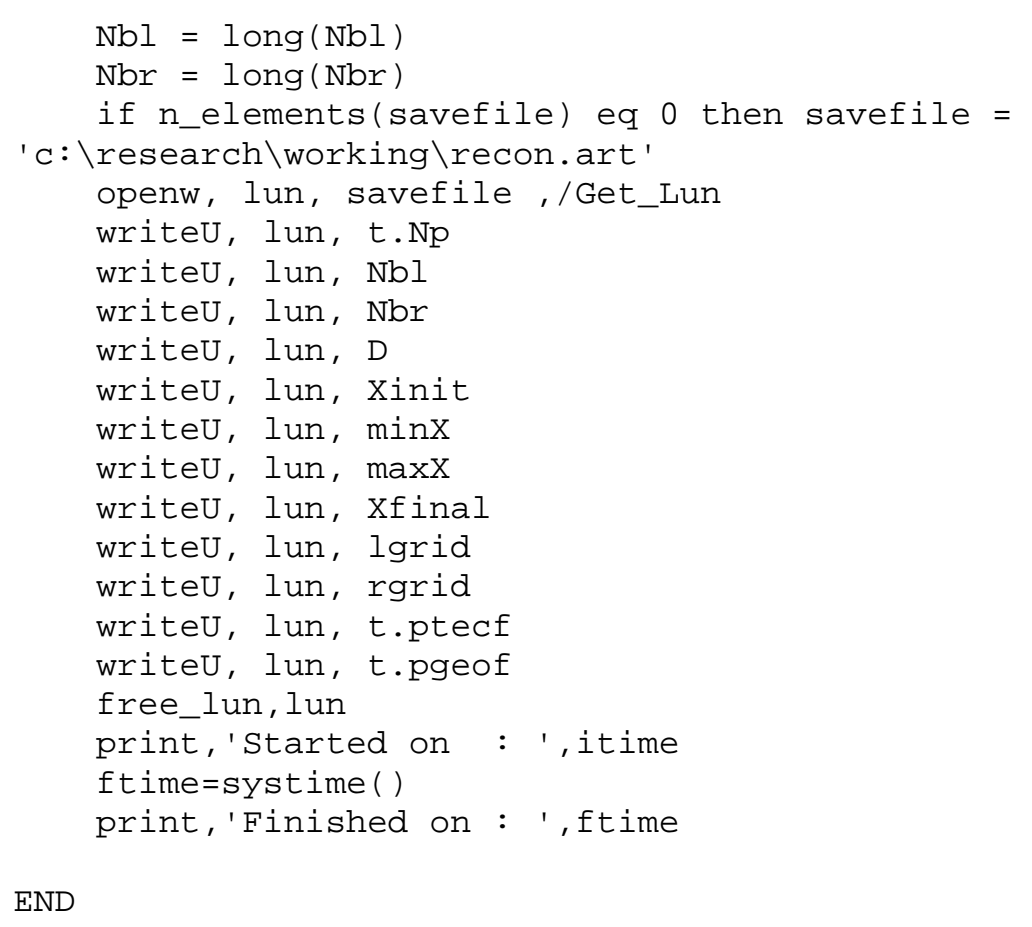

END 